\title{
Sulla riduzione delle singolarità di una superficie algebrica per mezzo di tra- sformazioni birazionali dello spazio.
}

\author{
(Di M. Pannelu, a Pavia.)
}

\section{Memoria Prima.}

\begin{abstract}
$L^{\prime}$ oggetto della presente Memoria, e delle altre che a questa faranno seguito, è la riduzione delle singolarità di una superficie algebrica per mezzo di trasformazioni birazionali dello spazio. Nel primo paragrafo viene studiata una speciale trasformazione cubica birazionale; nel secondo, un caso particolare di questa, e nel terzo, la trasformazione prodotto delle due precedenti. Le proprietà di questa ultima trasformazione potrebbero essere omesse; ma la loro conoscenza facilita alcune dimostrazioni, e di più le formole della trasformazione medesima sono utili per le ulteriori ricerche. Il paragrafo quarto è consacrato ad applicare la trasformazione stabilita nel secondo, ad una data superficie $F$ dell' ordine $n$, dotata di un punto $i$-plo, in cui il cono tangente è semplice e possiede una generatrice $j$-pla di natura qualsiasi, superficie alla quale è dapprima rivolto lo studio attuale. Infine nel paragrafo quinto viene applicata la trasformazione del primo, alla superficie $F^{\prime}$, trasformata di $F$.
\end{abstract}

\section{$\S 1$.}

1. Suppongasi di avere due spazi $\Sigma$ e $\Sigma^{\prime}$ e in essi prendansi due stelle di centri $O$ ed $O^{\prime}$, fra i raggi delle quali abbia luogo una trasformazione quadratica $\Omega$. Siano $a, b, c$ i raggi fondamentali della prima stella; $a^{\prime}, b^{\prime}, c^{\prime}$, quelli della seconda, in modo che ai raggi $a, b, c$, od $a^{\prime}, b^{\prime}, c^{\prime}$ corrispondono rispettivamente i piani $b^{\prime} c^{\prime}, c^{\prime} a^{\prime}, a^{\prime} b^{\prime} \quad 0 \quad b c, c a_{j} a b$. Inoltre prendansi nei 
due spazi anzidetti due fasci di piani di assi $s$ ed $s^{\prime}$, e fra questi stabiliscasi una proiettività II in guisa che ai piani $O s, \alpha, \beta$ del primo fascio corrispondano nel secondo i piani $O^{\prime} s^{\prime}, \alpha^{\prime}, \beta^{\prime}$, essendo $\alpha$ e $\beta$ due piani qualsivogliano dell'un fascio e $\alpha^{\prime}, \beta^{\prime}$ due piani qualsivogliano dell'altro. In tal maniera resta stabilita fra $i$ punti dei due spazi $\Sigma$ e $\Sigma^{\prime}$ una corrispondenza birazionale. Infatti, un punto qualunque $P$ di $\Sigma$ determina un raggio $r \equiv P O$ nella stella $(O)$ e un piano $\sigma \equiv P s$ nel fascio $(s)$. A quel raggio corrisponde in $\Omega$ un raggio $r^{\prime}$ della stella $\left(O^{\prime}\right)$, e a questo piano corrisponde in $\Pi$ un piano $\sigma^{\prime}$ del fascio $\left(s^{\prime}\right)$. Il raggio $r^{\prime}$ incontra il piano $\sigma^{\prime}$ in un punto $P^{\prime}$, che si considererà come il punto di $\Sigma^{\prime}$ corrispondente al punto $P$ dato in $\Sigma$. Viceversa ad un punto $P^{\prime}$ di $\Sigma^{\prime}$ corrisponde nello stesso modo, un punto $P$ in $\Sigma$. La trasformazione birazionale cosi individuata è precisamente quella che costituisce l'oggetto di questo primo paragrafo.

2. Innanzi tutto è facile trovare le formole di siffatta trasformazione. I piani $b c, c a, a b$ ed a prendansi come facce

$$
x_{1}=0 \quad x_{2}=0 \quad x_{3}=0 \quad x_{4}=0,
$$

del tetraedro fondamentale di $\Sigma$, e parimenti i piani $b^{\prime} c^{\prime}, c^{\prime} a^{\prime}, a^{\prime} b^{\prime}$ ed $\alpha^{\prime}$ come facce

$$
x_{1}^{\prime}=0 \quad x_{2}^{\prime}=0 \quad x_{3}^{\prime}=0 \quad x_{4}^{\prime}=0 \text {, }
$$

del tetraedro fondamentale di $\Sigma$ '. Per l'jpotesi che fra i raggi delle due stelle di centri

$$
O\left(x_{1}=x_{2}=x_{3}=0\right) \quad \text { ed } \quad O^{\prime}\left(x_{1}{ }^{\prime}=x_{2}{ }^{\prime}=x_{3}{ }^{\prime}=0\right),
$$

lia luogo una trasformazione quadratica, si ha intanto

$$
\begin{aligned}
& p x_{1}=x_{2}{ }^{\prime} x_{3}{ }^{\prime} \\
& p x_{2}=x_{3}{ }^{\prime} x_{1}^{\prime} \\
& p x_{3}=x_{1}{ }^{\prime} x_{2}^{\prime}
\end{aligned}
$$

essendo $\rho$ un fattore di proporzionalità. Inoltre siano

$$
p=a_{1} x_{1}+a_{2} x_{2}+a_{3} x_{3}=0, \quad p^{\prime}=a_{1}^{\prime} x_{1}^{\prime}+a_{2}^{\prime} x_{2}^{\prime}+a_{3}^{\prime} x_{3}^{\prime}=0,
$$

le equazioni dei due piani $O s$ ed $O^{\prime} s^{\prime}$; e quindi

$$
p=0, x_{4}=0 \text { e } p^{\prime}=0, x_{4}^{\prime}=0,
$$

quelle delle due rette $s$ ed $s^{\prime}$. Allora $\mathrm{i}$ due fasci di piani aventi per assi queste due rette hanno per equazioni

$$
p+\lambda x_{4} \equiv 0 \quad p^{\prime}+\lambda x_{4}^{\prime}=0
$$


dove $\lambda$ è un parametro variabile. E scritte così le loro equazioni, $\mathrm{i}$ fasci medesimi risultano proiettivi e in essi i piani $O s$ ed $O^{\prime} s^{\prime}$ sono corrispondenti.

Ciò stabilito, prendasi un punto $P^{\prime}$ nello spazio $\mathcal{L}^{\prime}$, ossia attribuiscasi alle variabili $x_{1}{ }^{\prime}, x_{2}{ }^{\prime}, x_{3}{ }^{\prime}, x_{4}{ }^{\prime}$ un sistema di valori particolari. Sostituiti questi valori nella seconda delle equazioni (2), resta determinato un valore del parametro $\lambda$, quel valore che dà il piano del fascio $\left(s^{\prime}\right)$ che passa per il punto $P^{\prime}$. Ponendo questo valore di $\lambda$ nella prima delle (2), si ottiene :

$$
p x_{4}^{\prime}-p^{\prime} x_{4}=0 \text {, }
$$

e questa è l'equazione del piano del fascio $(s)$ corrispondente nella proiettività $\Pi$ al piano anzidetto del fascio $\left(s^{\prime}\right)$. Ora, le prime tre coordinate del punto $P$ di $\Sigma$ corrispondente al punto $P^{\prime}$ preso in $\Sigma^{\prime}$ sono date dalle formole (1) e sostituiti $\mathrm{i}$ valori $\mathrm{di}$ tali coordinate alle variabili $x_{1}, x_{2}, x_{3}$, nella equazione precedente, si ottiene

donde

$$
\left(a_{1} x_{2}^{\prime} x_{3}{ }^{\prime}+a_{2} x_{3}{ }^{\prime} x_{1}^{\prime}+a_{3} x_{1}^{\prime} x_{2}\right) x_{4}^{\prime}-p^{\prime} \cdot \rho x_{4}=0,
$$

se per brevità si pone

$$
\rho x_{4}=\frac{q^{\prime} x_{i}^{\prime}}{p^{\prime}}
$$

$$
q^{\prime}=a_{1} x_{2}{ }^{\prime} x_{3}{ }^{\prime}+a_{2} x_{3}{ }^{\prime} x_{1}{ }^{\prime}+a_{3} x_{1}{ }^{\prime} x_{2}{ }^{\prime} .
$$

Quindi le coordinate del punto $P$ sono:

$$
\begin{aligned}
& \rho x_{1}=p^{\prime} x_{2}^{\prime} x_{3}^{\prime} \\
& \rho x_{2}=p^{\prime} x_{3}^{\prime} x_{1}^{\prime} \\
& \rho x_{3}=p^{\prime} x_{1}^{\prime} x_{2}^{\prime} \\
& \rho x_{4}=q^{\prime} x_{4}^{\prime} .
\end{aligned}
$$

Analogamente le formule che dànno le coordinate del punto $P^{\prime}$ di $\Sigma^{\prime}$ corrispondente ad un punto $P$ di $\Sigma$ sono:

$$
\begin{aligned}
& \rho^{\prime} x_{1}^{\prime}=p x_{2} x_{3} \\
& \rho^{\prime} x_{2}^{\prime}=p x_{3} x_{1} \\
& \rho^{\prime} x_{3}^{\prime}=p x_{1} x_{2} \\
& \rho^{\prime} x_{1}^{\prime}=q x_{1} .
\end{aligned}
$$

dove $\rho^{\prime}$ è un fattore di proporzionalità ed inoltre si è posto

$$
q=a_{1}^{\prime} x_{2} x_{3}+a_{2}^{\prime} x_{3} x_{1}+a_{3}^{\prime} x_{1} x_{2} \text {. }
$$


3. In virtù delle formule (3) si ha che ad un piano $V$ di $\Sigma$, condotto per il punto 0 , e che quindi ha per equazione

$$
v_{1} x_{1}+v_{2} x_{2}+v_{3} x_{3}=0,
$$

corrisponde in $\Sigma^{\prime}$ il luogo di cui l'equazione è

$$
p^{\prime}\left(v_{1} x_{2}{ }^{\prime} x_{3}{ }^{\prime}+v_{z} x_{3}{ }^{\prime} x_{1}{ }^{\prime}+v_{3} x_{1}{ }^{\prime} x_{2}{ }^{\prime}\right)=0,
$$

e che perciò si scinde nel piano fisso $O^{\prime} s^{t}$ e in un cono quadrico $I^{\prime}$. Questo cono circoscritto al triedro $O^{\prime} . a^{\prime} b^{\prime} c^{\prime}$ è appunto il cono della stella $\left(O^{\prime}\right)$ corrispondente nella trasformazione $\Omega$ al piano dato $V$. Prescindendo dal piano fisso $O^{\prime} s^{\prime}$, si ha dunque:

I. "Ad un piano $V$ di $\Sigma$ condotto per il punto $O$, corrisponde in $\Sigma^{\prime}$ " un cono quadrico $\Gamma^{\prime}$ circoscritto al triedro $O^{\prime} . a^{\prime} b^{\prime} c^{\prime}$."

In particolare al piano $O s$, di cui l'equazione è $p=0$, corrisponde il cono $C^{\prime}$, che ha per equazione $q^{\prime}=0$. Questo cono viene tagliato dal piano $O^{\prime} s^{\prime}$ in due rette, che si indicheranno con $d^{\prime}, e^{\prime}$.

Dal teorema precedente segue subito l'altro.

II. "Ad una retta di $\Sigma$ condotta per il punto $O$, corrisponde in $\Sigma^{\prime}$ "una retta passante per il punto $O^{\prime}$."

Sia

$$
u_{1} x_{1}+u_{2} x_{2}+u_{3} x_{3}+u_{4} x_{4}=0
$$

l'equazione di un piano $U$ di $\Sigma$. Per le formule (3), a questo piano corrisponde in $\Sigma^{\prime}$ una superficie $\varphi^{\prime}$ che ha per equazione

$$
\left(u_{1} x_{2}^{\prime} x_{3}^{\prime}+u_{2} x_{3}^{\prime} x_{1}^{\prime}+u_{3} x_{1}^{\prime} x_{2}^{\prime}\right) p^{\prime}+u_{4} q^{\prime} x_{4}^{\prime}=0,
$$

quindi la superficie $\varphi^{\prime}$ è intanto del $3 .^{\circ}$ ordine. Inoltre essa possiede in $O^{\prime}$ un punto doppio conico e il cono ivi tangente ha per equazione $q^{\prime}=0$, epperò è il cono fisso $C^{\prime}$. Infine contiene le rette $a^{\prime}, b^{\prime}, c^{\prime}, d^{\prime}, e^{\prime} s^{\prime}$, come è facile verificare. Dunque :

III. "Ad un piano qualunque $U$ di $\Sigma$ corrisponde in $\Sigma^{\prime}$ una super" ficie $\varphi^{\prime}$ del $3 .^{\circ}$ ordine, la quale possiede in $O^{\prime}$ un punto doppio conico, in " cui il cono tangente è il cono fisso $C^{\prime}$ e inoltre passa semplicemente per " le rette $a^{\prime}, b^{\prime}, c^{\prime}, d^{\prime}, e^{\prime}, s^{\prime} . "$

Quindi gli elementi fondamentali dello spazio $\Sigma^{\prime}$ sono il punto $O^{\prime}$ e le sei rette $a^{\prime}, b^{\prime}, c^{\prime}, d^{\prime}, e^{\prime}, s^{\prime}$ di cui le prime cinque concorrono in $O^{\prime}$ e $l^{\prime} u l-$ tima giace nel piano determinato dalla quarta e dalla quinta.

Una retta qualunque $R$ di $\Sigma$ può sempre essere considerata come l' intersezione di un piano $V$ che la proietta da $O$ con un piano $U$ passante per 
d' una superficie algebrica per mezzo di trasform. biraz. dello spazio. 71

essa. Al primo di questi due piani corrisponde in $\Sigma^{\prime}(I)$ un cono $\Gamma^{\prime}$; al secondo, (III), una superficie $\varphi^{\prime}$. Quel cono, di $2 .^{\circ}$ ordine, e questa superficie, del $3 .^{\circ}$, hanno in comune le tre rette semplici $a^{\prime}, b^{\prime}, c^{\prime}$; quindi s'intersecano, fuori di queste, secondo una cubica gobba $S^{\prime}$. - La completa intersezione delle due superficie deve possedere in $O^{\prime}$ un punto quadruplo, essendo $O^{\prime}$ un punto doppio per ciascuna delle superficie stesse; ma soltanto le rette $a^{\prime}, b^{\prime}, c^{\prime}$, passanti per $O^{\prime}$ fanno parte, come linee semplici, di questa intersezione, dunque la cubica $S^{\prime}$ passa per $O^{\prime}$. I due coni $\Gamma^{\prime}$ e $C^{\prime}$ hanno in comune le tre rette $a^{\prime}, b^{\prime}, c^{\prime}$; quindi s' intersecano ulteriormente secondo una retta passante per $O^{\prime}$, la quale è la tangente in questo punto alla curva $S^{\prime}$. Dunque la tangente alla curva $S^{\prime}$ nel punto $O^{\prime}$ è una generatrice del cono fisso $C^{\prime}$. - Il cono $\Gamma^{\prime}$ incontra la retta $s^{\prime}$, che appartiene alla superficie $\varphi^{\prime}$, in due punti, per i quali passa evidentemente la cubica $S^{\prime}$. - Il cono $\Gamma^{\prime}$ non contiene le due rette $d^{\prime}, e^{\prime}$; quindi la curva $S^{\prime}$ non si appoggia (in punti variabili) a nessuna di queste due rette. - Infine il piano tangente al cono $\Gamma^{\prime}$ lungo la retta $a^{\prime}\left(x_{2}^{\prime}=0, x_{3}^{\prime}=0\right)$ ha per equazione

$$
v_{3} x_{2}^{\prime}+v_{2} x_{3}^{\prime}=0
$$

e il piano tangente in un punto $\left(\alpha_{1}^{\prime}, o, o, \alpha_{1}^{\prime}\right)$ della medesima retta $a^{\prime}$ alla superficie $\varphi^{\prime}$ ha per equazione

$$
\left(u_{3} a_{1}^{\prime} \alpha_{1}^{\prime}+u_{4} a_{3} \alpha_{4}^{\prime}\right) x_{2}^{\prime}+\left(u_{2} u_{1}^{\prime} \alpha_{1}^{\prime}+u_{4} a_{2} \alpha_{4}^{\prime}\right) x_{3}^{\prime}=0 .
$$

Ora questo piano coincide con il precedente, se si ha:

$$
\begin{aligned}
& k v_{3}=u_{3} a_{1}^{\prime} \alpha_{1}^{\prime}+u_{4} a_{3} \alpha_{4}^{\prime} \\
& k v_{2}=u_{2} a_{1}^{\prime} \alpha_{1}^{\prime}+u_{4} a_{2} \alpha_{4}^{\prime},
\end{aligned}
$$

essendo $k$ un fattore di proporzionalità. Eliminando questo fattore, si ottiene

$$
\frac{\alpha_{1}^{\prime}}{\alpha_{4}^{\prime}}=\frac{u_{4}\left(a_{2} v_{3}-a_{3} v_{2}\right)}{a_{1}^{\prime}\left(v_{2} u_{3}-v_{3} u_{2}\right)}
$$

Dunque sopra la retta $a^{\prime}$ esiste un punto (quello che ba per coordinate $\mathrm{i}$ valori di $\alpha_{.1}{ }^{\prime}$ e o ${ }^{\prime}{ }^{\prime}$ ora determinati) in cui il piano tangente al cono $\Gamma^{\prime}$ coincide 'col piano tangente alla superficie $\varphi^{\prime}$. In questo punto le due superficie si toccano, quindi la loro completa intersezione deve ivi possedere un punto doppio, epperò la cubica $S^{\prime}$ passa per il punto medesimo. Riepilogando si ha dunque:

IV. "Ad una retta qualunque $R$ di $\Sigma$ corrisponde in $\Sigma^{\prime}$ una cubica " gobba $S^{\prime}$, la quale passa per il punto $O^{\prime}$ e tocea ivi il cono fisso $C^{\prime}$. Inoltre " essa si appoggia in due punti (variabili) alla retta $s^{\prime}$, in un punto (variabile) 
“ a ciascuna delle tre rette $a^{\prime}, b^{\prime}, c^{\prime}$ e in nessun punto (variabile) a ciascuna "delle due rette $d^{\prime}, e^{\prime}$."

La perfetta analogia delle costruzioni con le quali si trova il punto $P$

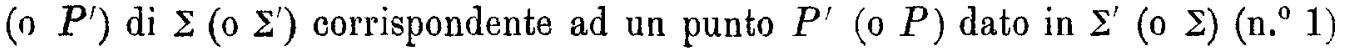
mostra che le proprieta mediante le quali si passa dal primo spazio al secondo servono ancora a passare da questo a quello. In particolare al piano $O^{\prime} s^{\prime}$ di $\Sigma^{\prime}$ corrisponde in $\Sigma$ un cono quadrico $C$, circoscritto al triedro $O . a b c$ e avente per equazione $q=0$. Questo cono viene tagliato dal piano $O s$ in due rette $d$ ed $e$, le quali (II) corrispondono rispettivamente alle rette $d^{\prime}$ ed $e^{\prime}$. Il punto $O$ e le rette $a, b, c, d, e, s$ sono gli elementi foudamentali di $\Sigma$. Inoltre teoremi affatto analoghi ai precedenti dànno le superficie $\varphi$ e le curve $S$ di $\Sigma$ corrispondenti ai piani $U^{\prime}$ e alle rette $R^{\prime}$ di $\Sigma^{\prime}$.

4. Ad una retta di $\Sigma$ condotta per $O$ corrisponde in $\Sigma^{\prime}$ (3. II) una retta passante per $O^{\prime}$. I due fasci proiettivi di piani $(s)$ ed $\left(s^{\prime}\right)$ determinano sopra queste due rette due punteggiate proiettive, due punti corrispondenti delle quali sono (1) punti corrispondenti della trasformazione birazionale fra i due spazi $\Sigma$ e $\Sigma^{\prime}$. Quindi, poichè al piano $O s$ del fascio $(s)$ corrisponde nella proiettivita $\Pi$ il piano $O^{\prime} s^{\prime}$ del fascio $\left(s^{\prime}\right)$, si ha:

I. " Ad un punto di $\Sigma$ infinitamente vicino ad $O$ corrisponde, in "generale, in $\Sigma^{\prime}$ un punto infinitamente vicino ad $O^{\prime}$."

Sia $P$ un punto qualunque del piano $O$ s. Poichè nella trasformazione $\Omega$, al piano $O s$ corrisponde il cono $C^{\prime}$; al raggio $P O$ corrisponde nella trasformazione stessa una generatrice $g^{\prime}$ di questo cono. Inoltre il punto $P$ determina nel fascio $(s)$ il piano $O s$, cui per la proiettività $\Pi$, corrisponde nel fascio $\left(s^{\prime}\right)$ il piano $O^{\prime} s^{\prime}$, il quale incontra quella generatrice $g^{\prime}$ nel punto $O^{\prime}$. Dunque ad un punto $P$ del piano $O s$ corrisponde in $\Sigma^{\prime}$ il punto $O^{\prime}$, o meglio, il punto $\omega^{\prime}$ infinitamente vicino ad $O^{\prime}$ sulla generatrice $g^{\prime}$. Questa generatrice non cambia quando il punto $P$ si muove descrivendo la retta $P O$ del piano $O s$; dunque ad ogni punto di questa retta corrisponde l'anzidetto punto $\omega$ '; epperò :

II. "Ad un punto $P$ del piano $O s$ di $\Sigma$ corrisponde, in generale, in " $\Sigma^{\prime}$ un punto $\omega^{\prime}$ infinitamente vicino ad $O^{\prime}$ sopra una determinata genera" trice del cono $C^{\prime}$. Questo punto $\omega^{\prime}$ non varia se il punto $P$ si muove de"scrivendo la retta $O P$ del piano $O s$."

In virtù di questo teorema applicato allo spazio $\Sigma^{\prime}$, si ha l'altro:

III. "Ad un punto w di $\Sigma$ infinitamente vicino ad $O$ e apparte* " nente al cono $C$ corrisponde in $\Sigma^{\prime}$ una retta passante per il punto $O^{\prime}$ e 
d'una superficie algebrica per mezzo di trasform. biraz. dello spazio. 73

" situata sul piano $O^{\prime} s^{\prime}$; e quindi al punto $O$ corrisponde, in questa maniera, "il piano $O^{\prime} s^{\prime}$. "

Sia $P$ un punto della retta $d$ (od $e$ ). Il raggio $P O$ coincide con $d($ od $e$ ), cui corrisponde nella trasformazione $\Omega$ la retta $d^{\prime}\left(\right.$ od $e^{\prime}$ ) (3). Il piano $P s$ coincide con $O s$ cui corrisponde nella proiettività $\Pi$ il piano $O^{\prime} s^{\prime}$. Ma questo piano contiene la retta $d^{\prime}$ (od $e^{\prime}$ ); dunque ogni punto di questa corrisponde al punto dato; epperò:

IV. " Ad ogni punto della retta $d$ od $e$ di $\Sigma$ corrisponde in $\Sigma^{\prime}$ la " retta $d$ od $e^{\prime} . "$

Sia $P$ un punto della retta $s$. Alla retta $P O$ corrisponde in $\Omega$ una generatrice $y^{\prime}$ nel cono $C^{\prime}$. Il piano che il punto $P$ somministra nel fascio $(s)$ è nel caso attuale indeterminato, cioè ogni piano di questo fascio può essere considerato come piano $P s$, al quale quindi corrisponde nella proiettività II ogni piano del fascio $\left(s^{\prime}\right)$. Ogni punto dell'anzidetta generatrice $g^{\prime}$ corrisponde dunque al punto datc $P$, eppero:

V. "Ad un punto $P$ della retta $s$ di $\Sigma$ corrisponde in $\Sigma^{\prime}$ una ge"neratrice del cono $C^{\prime}$, e quindi alla retta $s$ corrisponde il cono $C^{\prime}$."

Infine sia $P$ un punto della retta $a, o b, o c$, e per esempio della prima. Il raggio $P O$ coincide con $a$, cui corrisponde in $\Omega$ il piano $b^{\prime} c^{\prime}$. Al piano $P s$ del fascio $(s)$ corrisponde in $\Pi$ un piano del fascio $\left(s^{\prime}\right)$ il quale taglia il piano $b^{\prime} c^{\prime}$ secondo una retta appoggiata all'asse $s^{\prime}$ nel punto in cui l'asse medesimo incontra il piano $b^{\prime} c^{\prime}$. Questa retta corrisponde al punto dato $P$. Dunque:

VI. "Ad un punto $P$ della retta $a, \circ b, 0 c$, di $\Sigma$ corrisponde in " $\Sigma^{\prime}$ una retta del piano $b^{\prime} c^{\prime}$, o $c^{\prime} a^{\prime}$, o $a^{\prime} b^{\prime}$, appoggiata alla retta $s^{\prime}$; e quindi " alle rette $a, b, c$, corrispondono rispettivamente i piani $b^{\prime} c^{\prime}, c^{\prime} a^{\prime}, a^{\prime} b^{\prime}$. "

5 . Un piano di $\Sigma$ condotto per la retta $s$ ha per equazione

$$
p+\lambda x_{4}=0 \text {. }
$$

Quindi gli corrisponde in $\Sigma^{\prime}$ il loogo di cui l' equazione è

$$
q^{\prime}\left(p^{\prime}+\lambda x_{4}{ }^{\prime}\right)=0
$$

e che perciò si scinde nel cono $C^{\prime}$ e in un piano che è appunto il piano del fascio $\left(s^{\prime}\right)$ corrispondente nella proiettività $\Pi$ al piano dato nel fascio $(s)$. Prescindendo dal cono fisso $C^{\prime}$. che $(4, V)$ è l'ente geometrico di $\Sigma^{\prime}$ corrispondente alla retta $s$, si ba dunque:

I. " Ad un piano di $\Sigma$ condotto per la retta $s$ corrisponde in $\Sigma^{\prime}$ un "piano passante per la retta $s^{\prime}$." 
Una retta di $\Sigma$ appoggiata alla retta $s$ determina due piani: uno passante per $s$ e l'altro per $O$. Al primo corrisponde in $\Sigma^{\prime}(I)$ un piano passante per $s^{\prime}$; al secondo, $(3, I)$, un cono quadrico circoseritto al triedro $O^{\prime}$. $a^{\prime} b^{\prime} e^{\prime}$. Quindi alla retta data corrisponde la conica secondo cui quel piano taglia questo cono; epperò :

II. "Ad una retta di $\Sigma$ appoggiata alla retta $s$ corrisponde in $\Sigma$ ' " una conica appoggiata in due punti alla retta $s$ e in un punto a ciascuna " delle rette $a^{\prime} b^{\prime} c^{\prime}$."

Inoltre:

III. "Fra i punti di due piani corrispondenti di $\Sigma$ e di $\Sigma^{\prime}$ passanti " per le rette $s$ ed $s$, ha luogo una trasformazione quadratica, per la quale " sono fondamentali sopra ciascun piano i punti in cui questo incontra le rette " fondamentali della trasformazione $\Omega$ appartenente allo spazio in cui giace "il piano che si considera."

Come il teorema I si dimostra l'altro:

IV. "Ad un piano di $\Sigma$ condotto per una delle rette $a, b, c$ cor"corrisponde in $\Sigma$ ' un piano passante per la retta omologa."

Una retta di $\Sigma$ appoggiata ad a si può sempre riguardare come l'intersezione del pjano che la proietta da $a$ con un piano condotto ad arbitrio per essa. Al primo piano corrisponde in $\Sigma^{\prime}$ (IV) un piano passante per $a^{\prime}$; al se. condo $\left(3\right.$, III), una superficie del $3 .^{\circ}$ ordine $\varphi^{\prime}$, la quale contiene la retta $a^{\prime}$, epperò viene incontrata dall'anzidetto piano, oltre $a^{\prime}$, secondo una conica. Questa passa per $O^{\prime}$, perchè la completa intersezione delle due superficie deve possedere in $O^{\prime}$ un punto doppio. Inoltre tocea in $O^{\prime}$ il cono $C^{\prime}$, essendo questo il cono tangente in $O^{\prime}$ alla superficie $\varphi^{\prime}$. Infine si appoggia alla retta $s^{\prime}$ nel punto in cui questa incontra il piano in cui la conica è contenuta. Dunque:

V. "Ad una retta di $\Sigma$ appoggiata ad una delle rette fondamentali " $a, b, c$ corrisponde in $\Sigma^{\prime}$ una conica la quale passa per il punto $O^{\prime}$ e tocca "ivi il cono $C^{\prime}$, si appoggia in un altro punto, oltre $O^{\prime}$, alla retta $a^{\prime}$ o $l^{\prime}$ o $c^{\prime}$ "e infine si appoggia in un punto alla retta $s^{\prime}$."

Da questo teorema segue:

VI. "Fra i punti di due piani corrispondenti di $\Sigma$ e di $\Sigma^{\prime}$ passanti "per due rette omologhe $a$ e $a^{\prime}, 0 b$ e $b^{\prime}$, o $c$ e $c^{\prime}$, ha luogo una trasforma"zione quadratica, per la quale sono fondamentali sopra ciascun piano il punto " in cui questo incontra la retta $s$ o $s^{\prime}$, il punto $O$ od $O^{\prime}$, e il punto a questo " infinitamente vicino sulla generatrice che il cono $C,{ }^{\circ} C^{\prime}$, ha in comune "col piano che si considera, oltre la retta fondamentale per cui questo passa." 
Ad un piano di $\Sigma$ condotto per la retta $d$ corrisponde in $\Sigma^{\prime}$ un cono quadrico circoscritto al quadrispigolo $O^{\prime} \cdot a^{\prime} b^{\prime} c^{\prime} d^{\prime}$, mentre ad un piano qualunque $U$ corrisponde una superficie del $3 .^{\circ}$ ordine $\varphi^{\prime}$, la quale contiene le quattro rette $a^{\prime}, b^{\prime}, c^{\prime}, d^{\prime}$. Quindi questa superficie viene ulteriormente incontrata da quel cono secondo una conica, la quale si appoggia in un punto a ciascuna delle rette $a^{\prime}, b^{\prime}, c^{\prime}, d^{\prime}$ (nel punto in cui il cono e la superficie si toccano), e in un punto allia retta $s$ (nel punto in cui questa incontra il cono fuori della retta $d$ ). Questa conica è la curva di $\Sigma^{\prime}$ corrispondente all'intersezione dei due piani considerati in $\Sigma$, la quale è una retta appoggiata alla retta $d$. Dunque :

VII. "Ad una retta di $\Sigma$ appoggiata in un punto alla retta $d$, od $e$, " corrisponde in $\Sigma^{\prime}$ una conica, la quale si appoggia in un punto a ciascuna " delle rette $a^{\prime}, b^{\prime}, c^{\prime}, d^{\prime}$ (od $\left.e^{\prime}\right), s^{\prime}$."

Il piano $O s$ viene incontrato dai piani $b c, c a, a b$ in tre rette $l, m, n$ concorrenti in $O$. Si consideri un piano di $\Sigma$ che passi per una di queste tre rette, per esempio, per la prima. La sua equazione è

$$
p+\lambda x_{1}=0,
$$

essendo $\lambda$ un parametro variabile; quindi a quel piano corrisponde in $\Sigma^{\prime}$ il cono quadrico $\Gamma^{\prime}$ che ha per equazione

$$
q^{\prime}+\lambda x_{2}^{\prime} x_{3}^{\prime}=0,
$$

e che percio viene toceato lungo la retta $a^{\prime}\left(x_{2}^{\prime}=0, x_{3}^{\prime}=0\right)$ dallo stesso piano tangente lungo la retta medesima al cono $C^{\prime}\left(q^{\prime}=0\right)$. Questo cono $\Gamma^{\prime}$ viene tagliato da una superficie $\varphi^{\prime}$, fuori delle rette $a^{\prime}, b^{\prime}, c^{\prime}$, secondo una cubica gobba, la quale, per la proprietà ora osservata dei coni $\Gamma^{\prime}$ e $C^{\prime}$, tocca in $O^{\prime}$ la retta $a^{\prime}$. Inoltre la stessa cubica oscula in $O^{\prime}$ il piano di $\Sigma^{\prime}$ corrispondente al piano al di $\Sigma$. Dunque:

VIII. " $\Lambda$ d una retta di $\Sigma$ appoggiata ad una delle tre rette $l, m, n$ " corrisponde in $\Sigma^{\prime}$ una cubica gobba, la quale passa per il punto $O^{\prime}$ e tocca "ivi la retta $a^{\prime}$, o $b^{\prime}$, o $c^{\prime}$, ed oscula il piano di $\Sigma^{\prime}$ corrispondente al piano « $a l$, o $b l$, o $c l$ di $\Sigma$, si appoggia in due punti alla retta $s^{\prime}$, e in un punto " a ciascuna delle due rette $b^{\prime}$ e $c^{\prime}$, o $c^{\prime}$ e $a^{\prime}$, o $a^{\prime}$ e $b^{\prime}$." 


\section{\$2.}

6. Il caso particolare della trasformazione precedente che viene studiato in questo paragrafo, nasce supponendo che l'asse del fascio di piani (s) dello spazio $\Sigma$ si appoggi ad una, per esempio ad $a$, delle tre rette fondamentali della stella $(0)$. Perchè eiò avvenga basta supporre che il piano $0 s$ passi per la retta $a$, il che ha luogo se nella sua equazione si pone $a_{1}=0$, in modo che l'equazione stessa diventa

$$
p=a_{2} x_{2}+a_{3} x_{3}=0 .
$$

Il punto d'appoggio $A$ delle due rette $s$ ed $a$ è allora il vertice $x_{2}=x_{3}=x_{4}=0$ del tetraedro fondamentale di $\Sigma$. Inoltre, essendo $a_{1}=0$, si ha

ponendo:

$$
q^{\prime}=a_{2} x_{3}^{\prime} x_{1}^{\prime}+a_{3} x_{1}^{\prime} x_{2}^{\prime}=r^{\prime} x_{1}^{\prime},
$$

$$
r^{\prime}=a_{2} x_{3}^{\prime}+a_{3} x_{1}^{\prime} .
$$

Quindi le formule (3) e (4) diventano per il caso attuale:

(3)

$$
\left.\begin{array}{l}
\rho x_{1}=p^{\prime} x_{2}^{\prime} x_{3}^{\prime} \\
\rho x_{2}=p^{\prime} x_{3}^{\prime} x_{1}^{\prime} \\
\rho x_{3}=p^{\prime} x^{\prime} x_{1}^{\prime} \\
\rho x_{4}=r^{\prime} x_{1}^{\prime} x_{4}^{\prime}
\end{array}\right\}
$$

dove $p^{\prime}$ e $q$ hanno i significatí già loro attribuiti. Si noti infine che nello stesso caso il cono $C$ di $\Sigma$, corrispondente al piano $O^{\prime} s^{\prime}$ di $\Sigma^{\prime}$, viene tagliato dal piano $O s$ in due rette, una delle quali è la medesima retta $a$ e l'altra è una retta distinta da questa, che si chiamerà $d$; e che al piano $0 s(p=0)$ di $\Sigma$ corrisponde un luogo ohe si scinde nei due piani $r^{\prime}=0$ e $x_{1}^{\prime}=0$, il primo dei quali incontra il piano $O^{\prime} s^{\prime}$ in una retta $d^{\prime}$, che è la corrispondente di $d$. 
d'una superficie algebrica per mezzo di trasform. biraz. dello spazio.

7. Ad un piano $U^{\prime}$ di $\Sigma^{\prime}$ avente per equazione

$$
u_{1}^{\prime} x_{1}^{\prime}+u_{2}^{\prime} x_{2}^{\prime}+u_{3}^{\prime} x_{3}^{\prime}+u_{1}^{\prime} x_{4}^{\prime}=0
$$

corrisponde in $\Sigma$ una superficie $\varphi$ di cui l'equazione è :

$\left(a_{2} x_{2}+a_{3} x_{3}\right)\left(u_{1}^{\prime} x_{2} x_{3}+u_{2}^{\prime} x_{3} x_{1}+u_{3}^{\prime} x_{1} x_{2}\right)+u_{4}^{\prime}\left(a_{1}^{\prime} x_{2} x_{3}+a_{2}^{\prime} x_{3} x_{1}+a_{3}^{\prime} x_{1} x_{2}\right) x_{4}=0$.

Quindi, come nel caso generale, la superficie $\varphi$ è del $3 .^{\circ}$ ordine e possiede in $O$ un punto doppio conico in cui il cono tangente è il cono fisso $C$. Ma ora essa possiede un altro punto doppio conico nel punto $A$, in cui il cono tangente ha per equazione

$$
\left(a_{2} x_{2}+a_{3} x_{3}\right)\left(u_{2}^{\prime} x_{3}+u_{3}^{\prime} x_{2}\right)+u_{4}^{\prime}\left(a_{2}^{\prime} x_{3}+a_{3}^{\prime} x_{2}\right) x_{4}=0,
$$

e quindi è variabile col variare della superficie $\varphi$. Il piano tangente a questo cono e al precedente lungo la retta $a$ ha per equazione

$$
t=a_{3}^{\prime} x_{2}+a_{2}^{\prime} x_{3}=0 ;
$$

esso tocea la superficie $\varphi$ in ogni punto della medesima retta $a$ ed inoltre non cambia col cambiare di $\varphi$. Questa superficie infine contiene manifestamente le rette $b, c, d$ ed $s$. Dunque :

I. " Ad un piano qualunque $U^{\prime}$ di $\Sigma^{\prime}$ corrisponde in $\Sigma$ una super" ficie del $3 .^{\circ}$ ordine $\varphi$, la quale possiede due punti doppi conici: uno in $O$ " e l'altro in $A$. In quello il cono tangente è il cono fisso $C$; in questo, il " cono tangente è variabile. Inoltre essa tocca lungo la retta $a$ il piano fisso $t$ "e passa semplicemente per le rette $b, c, d$ ed $s . "$

Quindi gli elementi fondamentali dello spazio $\Sigma$ sono i due punti $O$ ed $A$, le rette $a \equiv O A, b, c, d$, concorrenti in 0 , la retta $e$ del piano $t$ infinitamente vicina ad $a$, e la retta $s$ uscente da $A$ è appoggiata in un punto $D$ alla retta $d$.

Come nel caso generale si ha qui :

II. " Ad un piano qualunque $V^{\prime}$ di $\Sigma^{\prime}$ condotto per il punto $O^{\prime}$ " corrisponde in $\Sigma$ un cono quadrico $\Gamma$ circoscritto al triedro $0 . a b c$."

Dai due teoremi precedenti è facile dedurre l'altro:

III. "Ad una retta qualunque $R^{\prime}$ di $\Sigma^{\prime}$ corrisponde in $\Sigma$ una cu" bica gobba $S$, la quale passa per i due punti $A$ ed $O$ e tocca in quest'ul" timo il cono fisso $C$. Inoltre essa si appoggia in un punto variabile, fuori "di $O$, a ciascuna delle rette $b$ e $c$, in un punto variabile, fuori di $A$, alla "retta $s$ e in nessun punto variabile alla retta $d$." 
Ed inoltre:

IV. "Ad una retta di $\Sigma$ ' condotta per $O^{\prime}$ corrispoude in $\Sigma$ una "retta passante per $0 . "$

8. Ad un piano $U$ di $\Sigma$ avente per equazione

$$
u_{1} x_{1}+u_{2} x_{2}+u_{3} x_{3}+u_{4} x_{4}=0,
$$

corrisponde in $\Sigma^{\prime}$ una superficie $\varphi^{\prime}$ di cui l'equazione è

$$
\left(u_{1} x_{2}^{\prime} x_{3}^{\prime}+u_{2} x_{3}^{\prime} x_{1}^{\prime}+u_{3} x_{1}^{\prime} x_{2}^{\prime}\right) p^{\prime}+u_{4} r^{\prime} x_{1}^{\prime} x_{4}^{\prime}=0 .
$$

Quindi la superficie $\varphi^{\prime}$, ancora del $3 .^{\circ}$ ordine, possiede in $O^{\prime}$ non più un punto doppio conico, come nel caso generale, ma un punto doppio biplanare, in cui $\mathrm{i}$ piani tangenti sono $\mathrm{i}$ piani fissi $r^{\prime}\left(r^{\prime}=0\right)$ e $b^{\prime} c^{\prime}\left(x_{1}^{\prime}=0\right)$. Inoltre essa contiene le rette secondo cui il piano $O^{\prime} s^{\prime}\left(p^{\prime}=0\right)$ taglia $\mathrm{i}$ tre piani $r^{\prime}$, $a^{\prime}\left(x_{4}^{\prime}=0\right)$ e $b^{\prime} c^{\prime}$, le prime due delle quali sono le rette $d^{\prime}$ ed $s^{\prime}$ e l'ultima è una retta che si dirà $e^{\prime}$; infine contiene pure le rette $a^{\prime}, b^{\prime}, c^{\prime}$. Dunque:

I. "Ad un piano qualunque $U$ di $\Sigma$ corrisponde in $\Sigma^{\prime}$ una superficie "del $3 .^{\circ}$ ordine $\varphi^{\prime}$, la quale possiede in $O^{\prime}$ un punto doppio biplanare, in cui " $\mathrm{i}$ piani tangenti sono $\mathrm{i}$ piani fissi $r^{\prime}$ e $b^{\prime} c^{\prime}$ e inoltre passa semplicemente "per le rette $a^{\prime}, b^{\prime}, c^{\prime}, d^{\prime}, e^{\prime}, s^{\prime} . "$

Quindi gli elementi fondamentali di $\Sigma^{\prime}$ sono il punto $O^{\prime}$ e le rette $a^{\prime}, b^{\prime}$, $c^{\prime}, d^{\prime}, e^{\prime}, s^{\prime}$, di cui le prime cinque concorrono in $\hat{O}^{\prime}$, mentre l'ultima si appoggia a $d^{\prime}$ e ad $e^{\prime}$ nei punti che si indicheranno con $D^{\prime}$ e $E^{\prime}$.

In particolare anche qui si ha:

II. "Ad un piano $V$ di $\cong$ condotto per $O$ corrisponde in $y^{\prime}$ un cono " quadrico $\Gamma^{\prime}$ circoscritto al triedro $O^{\prime}$. $a^{\prime} b^{\prime} c^{\prime} . "$

Inoltre sia

$$
w_{2} x_{2}+w_{3} x_{3}+w_{4} x_{4}=0,
$$

l'equazione di un piano $W$ di $s$ condotto per il punto $A$. A questo piano corrisponde in $z^{\prime}$ il luogo che ha per equazione

$$
\left[\left(w_{2} x_{3}^{\prime}+w_{3} x_{2}^{\prime}\right) p^{\prime}+w_{4} r^{\prime} x_{4}^{\prime}\right] x_{1}^{\prime}=0 \text {, }
$$

e che perciò si scinde nel piano $b^{\prime} c^{\prime}$ e in una quadrica $\Phi^{\prime}$, la quale tocca nel punto $O^{\prime}$ il piano $r^{\prime}$ e inoltre passa per le rette $a^{\prime}, d^{\prime}, s^{\prime}$. Dunque :

III. "Ad un piano $W$ di $\Sigma$ condotto per il punto $A$ corrisponde " in $\Sigma^{\prime}$ una quadrica $\Phi^{\prime}$, la quale passa per il punto $O^{\prime}$ e tocca ivi il piano " $r^{\prime}$ ed inoltre contiene le rette $a^{\prime}, d^{\prime}, s^{\prime} . "$ 
Una retta qualunque $R$ di $\Sigma$ può sempre essere considerata come l'intersezione di un piano $V$ condotto per $O$ con un piano $W$ condotto per $A$. A quel piano corrisponde in $\Sigma^{\prime}$ un cono $\Gamma^{\prime}$ (II); a questo, una quadrica $\Phi^{\prime}$ (III), la quale ha in comune con l'anzidetto cono la retta $a^{\prime}$. Quindi si riconosce immediatamente.

IV. "Ad una retta qualunque $R$ di $\Sigma$ corrisponde in $\Sigma^{\prime}$ una cubica " gobba $S$, la quale passa per il punto $O^{\prime}$ e tocca ivi il piano $r$ '. Inoltre si " appoggia in due punti variabili alla retta $s^{\prime}$, in un punto variabile, oltre $O^{\prime}$, " a ciascuna delle rette $a^{\prime}, b^{\prime}, c^{\prime} \mathrm{e}$ in nessun punto variabile a ciascuna delle "rette $d^{\prime}$ ed $e^{\prime} . "$

9. Il teorema I del n. ${ }^{0} 4$ rimane inalterato per l'attuale caso particolare. Lo stesso accade del teorema II, quando esso si applichi allo spazio s', mentre se si applica allo spazio $\Sigma$, poichè ora al piano $O s$ di $\Sigma$ corrisponde in $z^{\prime}(6)$ al piano $r^{\prime}$, si cambia nel seguente:

I. "Ad un punto $P$ del piano $O s$ di $\Sigma$ corrisponde, in generale, “un punto $w^{\prime}$ del piano $r^{\prime}$ infinitamente vicino ad $O^{\prime}$. Questo punto $w^{\prime}$ non "varia, se il punto $P$ si muove descrivendo il raggio $P O$ del piano $O$ s."

Quindi al teorema III del . $^{\circ} 4$, quando si parta dallo spazio $\Sigma^{\prime}$, si sostituisce l'altro:

II. " Ad un punto $w^{\prime}$ di $\Sigma^{\prime}$ infinitamente vicino ad $O^{\prime}$ e apparte" nente al piano $r$ corrisponde in $\subseteq$ una retta passante per il punto $O$ e si"tuata sul piano $O s$; e quindi al punto $O^{\prime}$ corrisponde, in questa maniera, "il piano Os."

Come il teorema IV dello stesso $n .^{\circ} 4$, si dimostra:

III. "Ad ogni punto della retta $d$, od $a$, di $\Sigma$ corrisponde in $\beth^{\prime}$ la " retta $d^{\prime}$ o $e^{\prime} . "$

Il teorema $\mathrm{V}$ del già citato $n^{\circ} 4$ resta immutato, se si riferisce allo spazio $\beth^{\prime}$; se invece si riferisce allo spazio $\Sigma$, diventa:

IV. "Ad ogni punto $P$ della retta $s$ di $\Sigma$ corrisponde in $\Sigma^{\prime}$ una : retta passante per il punto $O^{\prime}$ e situata sul piano $r^{\prime}$; e quindi alla retta $s$ "corrisponde il piano $r^{\prime}$."

Il teorema VI, applicato alla spazio :, vale soltanto per le due rette $b$ e $c$. altresì :

Infine dalla dimostrazione del toorema III del $n .^{\circ}$ precedente risulta

V. "Al punto fondamentale $A$ di $\Sigma$ corrisponde in $\Sigma^{\prime}$ il piano $b^{\prime} c^{\prime}$. " 10. I teoremi I e IV del n. 5 valgono anche nel caso particolare ora in esame. Ciò osservato, si consideri una retta $R$ di ¿ condotta per il 
punto $A$. Essa determina due piani, uno passante per la retta $s$ e l'altro per la retta $a$, ai quali, per l'osservazione fatta, corrispondono in $\Sigma$ ' rispettivamente un piano passante per $s$ e l'altro per $a$. Questi ultimi due piani si tagliano secondo una retta $R$, appoggiata alle due rette $s^{\prime}$ ed $a^{\prime}$. Dunque:

I. "Ad una retta di $\Sigma$ condotta per il punto $A$ corrisponde in $\Sigma^{\prime}$ " una retta appoggiata alle due rette $s^{\prime}$ ed $a^{s}$, e viceversa."

Per il teorema $\mathrm{V}$ del $n .^{\circ}$ precedente si hả che ad ogni punto di $\Sigma$ infinitamente vicino ad $A$ corrisponde in $\aleph^{\prime}$ un punto del piano $b^{\prime} c^{\prime}$. Ora per il teorema testè dimostrato si può dire più precisamente. Ad ogni punto $P$ di $z$ infinitamente vicino ad $A$ corrisponde in $2^{\prime}$ il punto $P^{\prime}$ in cui il piano $b^{\prime} c^{\prime}$ viene incontrato dalla retta $R^{\prime}$ di $\Sigma^{\prime}$ corrispondente alla retta $R$ di $\Sigma$ passante per $A$ e individuata dal punto dato $P$. Viceversa ad ogni punto $P^{\prime}$ di $\Sigma^{\prime}$ preso sopra il piano $b^{\prime} c^{\prime}$, corrisponde in $\Sigma$ il punto $P$ infinitamente vicino ad $A$ sulla retta $R$ passante per $A$ e corrispondente alla retta $\Sigma^{\prime}$ condotta per il punto $P^{\prime}$ ed appoggiata alle due rette $s^{\prime}$ ed $a^{\prime}$.

Se al punto $P$ infinitamente vicino ad $A$ si sostituisce la retta $R$ che lo congiunge ad $A$, la corrispondenza precedente può intendersi stabilita fra i punti del piano $b^{\prime} c^{\prime}$ e le rette della stella $(A)$. Le formule mediante le quali in questa corrisponderiza si passa da un punto $P^{\prime}$ del piano $b^{\prime} c^{\prime}$ alla retta corrispondente $R$ della stella $(A)$, sono in virtù delle (5), le seguenti :

$$
\begin{aligned}
& \rho x_{2}=\left(a_{2}^{\prime} x_{2}^{\prime}+a_{3}^{\prime} x_{3}^{\prime}\right) x_{3}^{\prime} \\
& \rho x_{3}=\left(a_{2}^{\prime} x_{2}^{\prime}+a_{3}^{\prime} x_{3}^{\prime}\right) x_{2}^{\prime} \\
& \rho x_{4}=\left(a_{3} x_{2}^{\prime}+a_{2} x_{3}^{\prime}\right) x_{4}^{\prime},
\end{aligned}
$$

nelle quali $x_{2}, x_{3}, x_{4}$ si riguardino come le coordinate di una retta della stella $(A)$. Risolvendo queste equazioni rispetto $x_{2}^{\prime}, x_{3}^{\prime}, x_{4}^{\prime}$ si ottengono le formule

$$
\begin{aligned}
& \rho^{\prime} x_{2}^{\prime}=\left(a_{2} x_{2}+a_{3} x_{3}\right) x_{3} \\
& \rho^{\prime} x_{3}^{\prime}=\left(a_{2} x_{2}+a_{3} x_{3}\right) x_{2} \\
& \rho^{\prime} x_{4}^{\prime}=\left(a_{3}^{\prime} x_{2}+a_{2}^{\prime} x_{3}\right) x_{4},
\end{aligned}
$$

che servono al passaggio inverso.

Quindi ad un piano $W$ della stella $(A)$ corrisponde sul piano $b^{\prime} c^{\prime}$ una conica che tocca nel punto $O^{\prime}$ la retta $a_{1}$ di equazione

$$
a_{3} x_{2}^{\prime}+a_{2} x_{3}^{\prime}=0 \text {, }
$$


d'una superficie algebrica per mezzo di trasform. biraz. dello spazio. $\quad \$ 1$

e che passa per il punto $E^{\prime}$, intersezione delle rette, le cui equazioni sono:

$$
a_{2}^{\prime} x_{2}^{\prime}+a_{3}^{\prime} x_{3}^{\prime}=0, \quad x_{4}^{\prime}=0 \text {. }
$$

Quella conica è l'intersezione del piano $b^{\prime} c^{\prime}$ con la quadrica $\Phi^{\prime}$ di $\Sigma^{\prime}$ corrispondente $(8, \mathrm{III})$ al piano $W$ condotto per $A$. La retta $a_{1}$ è l'intersezione dei due piani $r^{\prime}$ e $b^{\prime} c^{\prime}$ e il punto $E^{\prime}$ è il punto d'incontro della retta $s^{\prime}$ col piano $b^{\prime} c^{\prime}$.

Così ad una retta del piano $b^{\prime} c^{\prime}$ corrisponde nella stella $(A)$ un cono quadrico, che tocca lungo la retta $a$ il piano fisso $t$ di equazione

$$
a_{3}^{\prime} x_{2}+a_{2}^{\prime} x_{3}=0
$$

e che passa per la retta $s$. Questo cono è quello che nel punto $A$ tocca la superficie $\varphi$ di $\Sigma$ corrispondente $(7, \mathrm{I})$ ad un piano di $\Sigma^{\prime}$ condotto per la retta data sul piano $b^{\prime} c^{\prime}$.

Riepilogando si ha dunque:

II. "Fra le rette della stella $(A)$ e i punti del piano $b^{\prime} c^{\prime}$ ha luogo " una trasformazione quadratica. I punti fondamentali del piano sono $E^{\prime}, O^{\prime}$ $\because$ e il punto a questo infinitamente vicino sulla retta $a_{1}$; le rette fondamen" tali della stella sono $s, a$ e la retta a questa infinitamente vicino sul "piano $t$."

Inoltre come $\mathrm{i}$ teoremi II, $\mathrm{V}$ e VII del $\mathrm{n}^{\circ} 5$ si dimostrano $\mathrm{i}$ seguenti: III. " Ad una retta di $\Sigma$ appoggiata alla retta $s$ corrisponde in $\Sigma^{\prime}$ " una conica appoggiata in due punti alla retta $s^{\prime}$ e in un punto a ciascuna " delle rette $a^{\prime}, b^{\prime}, c^{\prime}$."

IV. "Ad una retta di $\Sigma$ appoggiata alla retta a corrisponde in $\Sigma^{t}$ " una conica, la quale passa per il punto $O^{\prime}$ e tocea ivi il piano $b^{\prime} c^{\prime}$, si ap" poggia in un punto variabile, oltre $O^{\prime}$, alla retta $a^{\prime}$ e in un punto varia. "bile alla retta $s^{\prime} . "$

V. "Ad una retta di $\Sigma$ appoggiata alla retta $b$, o $c$, corrisponde in " $\Sigma^{\prime}$ una conica, la quale passa per il punto $O^{\prime}$ e tocea ivi il piano $r^{\prime}$, si " appoggia in un punto variabile, oltre $O^{\prime}$, alla retta $b^{\prime} \quad c^{\prime}, 0$ in un punto "variabile alla retta $s^{\prime}$."

VI. "Ad una retta di $\Sigma$ appoggiata alla retta $d$ corrisponde in $\Sigma^{\prime}$ " una conica appoggiata in un punto a ciascuna delle rette $a^{\prime} b^{\prime} c^{\prime} d^{\prime} s^{\prime}$."

11. In modo analogo si hanno gli altri teoremi;

I. "Ad una retta di $\Sigma^{\prime}$ appoggiata alla retta $s^{\prime}$ corrisponde in $\Sigma$ una " conica, la quale passa per il punto $A$, si appoggia alla retta $s$ in un Annali di Matematica, tomo XXV. 
"altro punto variabile, oltre $A$, e in un punto a ciascuna delle due rette " $b$ e $c . "$

II. "Ad una retta di $\Sigma^{\prime}$ appoggiata alla retta $a^{\prime}$ corrisponde in $\Sigma$ " uṇa conica, la quale passa per i punti $A$ ed $O$, e tocca in quest'ultimo il " cono C."

III. : Ad una retta di $\Sigma^{\prime}$ appoggiata alla retta $b^{\prime}$ o $c^{\prime}$, corrisponde " in $\Sigma$ una conica, la quale passa per il punto $O$ e tocca ivi il cono $C$, si ap" poggia in un punto variabile, oltre $O$, alla retta $b, o c$, e in fine si ap"poggia in un punto variabile alla retta s."

IV. "Ad una retta di $\Sigma^{\prime}$ appoggiata alla retta $d^{\prime}$ corrisponde in $\Sigma$ " una conica, la quale passa per il punto $A$ e si appoggia a ciascuna delle "rette $b, c, d . "$

V. "Ad una retta di $\Sigma^{\prime}$ appoggiata alla retta $e^{\prime}$ corrisponde in $\Sigma$ " una conica, la quale si appoggia a ciascuna delle rette $a, b, c, s$ e nel suo "punto d'appoggio con a tocea il piano fisso $t$."

VI. "Ad una retta di $\Sigma^{\prime \prime}$ appoggiata alla retta $a_{1}$, intersezione dei " due piani $r^{\prime}$ e $b^{\prime} c^{\prime}$, corrisponde in $\Sigma$ una cubica gobba la quale passa per "i punti $A$ ed $O$, tocea in $A$ la retta $s$ e in $O$ il cono $C$ e si appoggia in "un punto variabile a ciascuna delle rette $b$ e $c . "$

Quest'ultima proprietà si rende subito manifesta osservando prima che ad ogni piano di $\Sigma^{\prime}$ passante per la retta $a_{1}$ corrisponde in $\Sigma$ un cono quadrico circoscritto al triedro $O . a b c$ e tangente lungo la retta $a$ al piano $O s$; e poi considerando una retta appoggiata ad $a_{1}$ come l'intersezione del piano che la proietta dalla stessa $a_{1}$ con un piano qualunque condotto per essa.

12. In virtù del teorema III del $n .^{\circ} 10$ e del teorema I del $n .^{\circ}$ precedente, si ha:

I. "Fra i punti di due piani corrispondenti $\sigma$ e $\sigma$ di $\Sigma$ e $\Sigma^{\prime}$ pas"santi per le rette $s$ ed $s^{\prime}$, ha luogo una trasformazione quadratica, per la "quale sono fondamentali sul piano $\sigma$ il punto $A$ e i punti in cui esse in" contra le rette $b$ e $c$, e sul piano $\sigma^{\prime} \mathrm{i}$ punti in cui questo incontra le tre "rette $a^{\prime}, b^{\prime}, c^{\prime}$."

Da questo teorema segue immediatamente:

II. "Ad una retta di $\Sigma^{\prime}$ condotta per il punto $D^{\prime}$ corrisponde in $\Sigma$ " una conica passante per i punti $A$ e $D$ e appoggiata alle rette $b$ e $c . "$

III. "Ad una retta di $\Sigma$ ' condotta per il punto $E^{\prime}$ corrisponde in $\Sigma$ " una conica che passa per il punto $A$ e tocca ivi il piano $t$, si appoggia 
d' unu superficie algebrica per mezzo di trasform. biraz. dello spazio. 83

" in un altro punto, oltre $A$, alla retta $s$ e in un punto a ciascuna delle due "rette $b$ e $c$."

Il teorema IV del n. ${ }^{0} 10$ e quello II del $n .^{0} 11$ somministrano l'altro: IV. " Fra i punti di due piani corrispondenti $\alpha$ ed $\alpha^{\prime}$ di $\boldsymbol{\Sigma}$ e di $\boldsymbol{\Sigma}^{\prime}$ " passanti rispettivamente per le rette $a$ ed $a^{\prime}$, ha luogo una trasformazione "quadratica, per la quale sono fondamentali sul piano a i punti $A, O$ e il "punto a questo infinitamente vicino sulla generatrice che il cono $C$ ha in " comune col piano $\alpha$, oltre la retta $a$; e sul piano $\alpha$, il punto in cui questo " incontra la retta $s^{\prime}$, il punto $O^{\prime}$ e il punto a questo infinitamente vicino " sulla retta secondo la quale il piano $\alpha$.taglia il piano $b^{\prime} c^{\prime}$."

Analogamente il teorema $\mathrm{V}$ del $n .^{\circ} 10$ e quello III del n. 11 dànno:

V. "Fra $i$ punti di due piani corrispondenti $\beta$ e $\beta$, o $\gamma$ e $\gamma^{\prime}$, di $\Sigma$ "e di $\Sigma^{\prime}$ passanti rispettivamente per le rette $b$ e $b^{\prime}$, o $c$ e $c^{\prime}$, ha luogo una " trasformazione quadratica, per la quale sono fondamentali sul piano $\beta, 0 \gamma$, " il punto in cui questo incontra la retta $s$, il punto $O$ e il punto a questo "infinitamente vicino sulla generatrice che il cono $C$ ha in comune col piano " $\beta$, , $\gamma$, oltre la retta $b$ o $c$; e sul piano $\beta^{\prime} \circ \gamma^{\prime}$, il punto in cui questo in" contra la retta $s^{\prime}$, il punto $O^{\prime}$ e il punto a questo infinitamente vicino sulla "retta d'intersezione del piano $\beta^{\prime}, 0 \gamma^{\prime}$, col piano $r^{\prime}$."

In particolare, ai piani $b d, c d$ e $t$ di $\Sigma$ corrispondono in $\Sigma^{\prime}$ rispettivamente i piani $b^{\prime} d^{\prime}, c^{\prime} l^{\prime}$ e $a^{\prime} e^{\prime}$; ed è facile vedere che ad una retta di uno qualunque di quei piani corrisponde una retta situata sul piano corrispondente. Quindi:

VI. "Fra i punti dei due piani corrispondenti $b d$ e $b^{\prime} d^{\prime}$, o $c d$ e " $c^{\prime} d^{\prime}$ o $t$ e $a^{\prime} e^{\prime}$ di $\Sigma$ e di $\Sigma^{\prime}$ ha luogo una corrispondenza omografica."

Dato in $\Sigma^{\prime}$ un piano $U^{\prime}$, resta sopra questo determinata la retta $T$, che si appoggia alle due rette fondamentali $a^{\prime}$ ed $s^{\prime}$, alla quale eorrisponde in $\Sigma$ $(10, I)$ una retta $T$ passante per il punto $A$. Ogni retta $R^{\prime}$ del piano $U^{\prime}$ appoggiata alla retta $d^{\prime}$ incontra in un punto la retta $T^{\prime}$; quindi la conica di \& corrispondente $(11,1 V)$ a quelia retta $R^{\prime}$ si appoggia in un punto variabile alla retta $T$. Inoltre la conica medesima passa per il punto $A$ e tocca ivi il cono tangente alla superficie $\varphi$ corrispondente al piano $U^{\prime}$. Infine essa si appoggia alle rette $b, c, d$. Dunque:

VII. " Alle rette di $\Sigma^{\prime}$ appoggiate in un medesimo punto alla retta $d^{\prime}$ "e situate in uno stesso piano, corrispondono in $\Sigma$ le coniche appoggiate alle "rette $b, c, d$, passanti per il punto $A$ e ivi tangenti ad un cono determi" nato col piano dato e situate sui piani di un fascio avente per asse una retta "passante per $A$ e parimenti determinata." 
Dato in $\Sigma^{\prime}$ un piano $U^{\prime}$, resta sopra questo determinata la conica $\delta^{\prime}$ che si appoggia alle cinque rette $a^{\prime}, b^{\prime}, c^{\prime}, d^{\prime}, s^{\prime}$, alla quale corrisponde in $\mathbf{s}$ una retta $\delta$, perchè quella conica viene incontrata in un sol punto variabile dalla superficie del $3 .^{\circ}$ ordine $\varphi^{\prime}$ di $\Sigma^{\prime}$ corrispondente ad un piano qualunque di $\Sigma$. Ogni 'retta $R^{\prime}$ del piano $U^{\prime}$ appoggiata alla retta $e^{\prime}$, incontra in due punti la conica $\partial^{\prime}$, quindi la conica di $\Sigma$ corrispondente $(11, \mathrm{~V})$ a quella retta $R^{\prime}$ si appoggia in due punti variabili alla retta $\delta$. Ricordando le altre proprietà della conica stessa, si ha dunque:

VIII. " Alle rette di $\Sigma^{\prime}$ appoggiate in un medesimo punto alla retta $e^{\prime}$ " e situate in uno stesso piano, corrispondono in $\Sigma$ le coniche appoggiate " alle rette $a, b, c, s$, tangenti nei loro punti d'appoggio con $a$ al piano " fisso $t$ e situate sui piani di un fascio avente per asse una retta determi"nata dal piano dato. $n$

\section{$\S 3$.}

13. Supposto che fra gli spazi $\Sigma$ e $\Sigma^{\prime}$ abbia luogo la trasformazione studiata nel paragrafo precedente, stabiliscasi fra questo ultimo e un terzo spazio $\mathbf{\Sigma}^{\prime \prime}$ la trasformazione del paragrafo primo.

Come asse del fascio fondamentale di piani dello spazio $\Sigma^{\prime}$, prendasi ancora la retta $s^{\prime}$ di cui le equazioni sono

$$
p^{\prime}=a_{1}^{\prime} x_{1}^{\prime}+a_{2}^{\prime} x_{2}^{\prime}+a_{3}^{\prime} x_{3}^{\prime}=0, \quad x_{4}^{\prime}=0,
$$

e come asse del fascio fondamentale di piani di $\Sigma^{\prime \prime}$, la retta $s^{\prime \prime}$ che ha per equazioni

$$
p^{\prime \prime}=a^{\prime \prime}{ }_{1} x_{1}{ }_{1}+a^{\prime \prime}{ }_{2} x^{\prime \prime}{ }_{2}+a^{\prime \prime}{ }_{3} x^{\prime \prime}{ }_{3}=0, \quad x^{\prime \prime}=0 \text {. }
$$

Poichè questi due fasci di piani debbono essere proiettivi fra loro, e al piano $O^{\prime} s^{\prime}\left(p^{\prime}=0\right)$ del primo deve corrispondere il piano $O^{\prime \prime} s^{\prime \prime}\left(p^{\prime \prime}=0\right)$ del secondo, le loro equazioni possono essere scritte cos̀ :

$$
p^{\prime}+\lambda x^{\prime}=0, \quad p^{\prime \prime}+\lambda x^{\prime \prime}{ }_{4}=0,
$$

dove $\lambda$ rappresenti un parametro variabile.

Come centro della stella fondamentale di $\Sigma^{\prime}$ si scelga ancora il punto $O^{\prime}$, di coordinate $x_{1}^{\prime}=x_{2}^{\prime}=x_{3}^{\prime}=0$; e come rette fondamentali di questa stella, la retta $a_{1}$, intersezione dei due piani

$$
x^{\prime}{ }_{1}=0, \quad r^{\prime}=a_{3} x_{2}^{\prime}+a_{2} x_{3}^{\prime}=0,
$$


e altre due rette $b_{1}$ e $c_{\mathrm{t}}$ condotte ad arbitrio per $a^{\prime}$. Le coordinate della retta $a_{1}$ sono: $0, a_{2},-a_{3}$; quelle delle rette $b_{1}$ e $c_{1}$ dicansi rispettivamente $b_{1}^{\prime}, b_{2}^{\prime}, b_{3}^{\prime}$ e $c_{1}^{\prime}, c_{2}^{\prime}, c_{3}^{\prime}$. Poichè le tre rette $a_{1}, b_{1}, c_{1}$ non possono giacere sullo stesso piano, il determinante

$$
\left|\begin{array}{ccc}
0, & a_{2}, & -a_{3} \\
b_{1}^{\prime} & b_{2}^{\prime} & b_{3}^{\prime} \\
e^{\prime}{ }_{1} & c_{2}^{\prime} & c_{3}^{\prime}
\end{array}\right|
$$

è necessariamente diverso da zero, e quindi è tale anche il suo reciproco

$$
H^{\prime}=\left|\begin{array}{lll}
\alpha_{1}^{\prime} & \alpha_{2}^{\prime} & \alpha_{3}^{\prime} \\
\beta_{1}^{\prime} & \beta_{2}^{\prime} & \beta_{3}^{\prime} \\
\gamma_{1}^{\prime} & \gamma_{2}^{\prime} & \gamma_{3}^{\prime}
\end{array}\right|
$$

i cui elementi sono i complementi algebrici di quelli del determinante precedente.

Come centro della stella fondamentale di $\Sigma^{\prime \prime}$ assumasi il punto $O^{\prime \prime}$ di coordinate $x^{\prime \prime}{ }_{1}=x^{\prime \prime}{ }_{2}=x^{\prime \prime}{ }_{3}=0$, e (some rette fondamentali della stella medesima, le rette $a_{1}^{\prime}, b_{1}^{\prime}, c_{1}^{\prime}$, intersezioni dei piani $x^{\prime \prime}{ }_{2}=0$ e $x^{\prime \prime}{ }_{3}=0, x^{\prime \prime}{ }_{3}=0$ e $x_{1}^{\prime \prime}=0, x_{1}^{\prime \prime}=0$ e $x^{\prime \prime}{ }_{2}=0$ rispettivamente.

Per determinare la corrispondenza quadratica che deve aver luogo fra i raggi delle due stelle $\left(O^{\prime}\right)$ ed $\left(O^{\prime \prime}\right)$, basta dare una coppia di raggi corrispondenti $m^{\prime}$ ed $m^{\prime \prime}$. Se $\left(m_{1}^{\prime}, m_{2}^{\prime}, m_{3}^{\prime}\right)$ e $\left(m_{1}^{\prime \prime}, m^{\prime \prime}{ }_{2}, m^{\prime \prime}{ }_{3}\right)$ sono le coordinate di questi due raggi, poichè tre qualsivogliano delle quattro rette $m^{\prime}, a_{1}, b_{1}, c_{1}$ o $m^{\prime \prime}, a_{1}^{\prime}, b_{1}^{\prime}, c_{1}^{\prime}$ non possono essere situate sullo stesso piano, i determinanti

$$
\begin{gathered}
A^{\prime}=\left|\begin{array}{ccc}
m_{1}^{\prime} & m_{2}^{\prime} & m_{3}^{\prime} \\
b_{1}^{\prime} & b_{2}^{\prime} & b_{3}^{\prime} \\
c_{1}^{\prime} & c_{2}^{\prime} & c_{3}^{\prime}
\end{array}\right|, \quad B^{\prime}=\left|\begin{array}{ccc}
m_{1}^{\prime} & m_{2}^{\prime} & m_{3}^{\prime} \\
c_{1}^{\prime} & c_{2}^{\prime} & c_{3}^{\prime} \\
0 & a_{2} & -a_{3}
\end{array}\right|, \\
C^{\prime}=\left|\begin{array}{ccc}
m_{1}^{\prime} & m_{2}^{\prime} & m_{3}^{\prime} \\
0 & a_{2} & -a_{3} \\
b_{1}^{\prime} & b_{2}^{\prime} & b_{3}^{\prime}
\end{array}\right|,
\end{gathered}
$$

come le coordinate $m_{1}^{\prime \prime}, m_{2}^{\prime \prime}, m^{\prime \prime}{ }_{3}$, debbono essere diversi da zero. formule.

Determinata così la trasformazione quadratica, è facile trovarne le 
I piani $b_{1} a_{1}$ e $b_{1} c_{1}$ della stella $(0)$ hanno per equazioni

$$
\left|\begin{array}{ccc}
x_{1}^{\prime} & x_{2}^{\prime} & x_{3}^{\prime} \\
0 & a_{2} & -a_{3} \\
b_{1}^{\prime} & b_{2}^{\prime} & b_{3}^{\prime}
\end{array}\right|=0, \quad\left|\begin{array}{lll}
x_{1}^{\prime} & x_{2}^{\prime} & x_{3}^{\prime} \\
b_{1}^{\prime} & b_{2}^{\prime} & b_{3}^{\prime} \\
c_{1}^{\prime} & c_{2}^{\prime} & c_{3}^{\prime}
\end{array}\right|=0,
$$

ossia

$$
x_{1}^{\prime} \gamma_{1}^{\prime}+x_{2}^{\prime} \gamma_{2}^{\prime}+x_{3}^{\prime} \gamma_{3}^{\prime}=0, \quad x_{1}^{\prime} \alpha_{1}^{\prime}+x_{2}^{\prime} \alpha_{2}^{\prime}+x_{3}^{\prime} \alpha_{3}^{\prime}=0 \text {, }
$$

e quindi l'equazione di un piano qualunque del fascio che ha per asse la retta $b_{1}$, ̀̀

$$
\left(x_{1}^{\prime} \gamma_{1}^{\prime}+x_{2}^{\prime} \gamma_{2}^{\prime}+x_{3}^{\prime} \gamma_{3}^{\prime}\right)+\mu\left(x_{1}^{\prime} \alpha_{1}^{\prime}+x_{2}^{\prime} \alpha_{2}^{\prime}+x_{3}^{\prime} \alpha_{3}^{\prime}\right)=0,
$$

dove $\mu$ rappresenti un parametro variabile. I valori che prende questo parametro, quando il piano del fascio passa per la retta data $m^{\prime}$ o per un'altra retta $n$ sono:

$$
\begin{gathered}
\mu=\mu_{1}=-\frac{m_{1}^{\prime} \gamma_{1}^{\prime}+m_{2}^{\prime} \gamma_{2}^{\prime}+m_{3}^{\prime} \gamma_{3}^{\prime}}{m_{1}^{\prime} \alpha_{1}^{\prime}+m_{2}^{\prime} \alpha_{2}^{\prime}+m_{3}^{\prime} \alpha_{3}^{\prime}}=-\frac{C^{\prime}}{A^{\prime}} \\
\mu=\mu_{2}=-\frac{x_{1}^{\prime} \gamma_{1}^{\prime}+x_{2}^{\prime} \gamma_{2}^{\prime}+x_{3}^{\prime} \gamma_{3}^{\prime}}{x_{1}^{\prime} \alpha_{1}^{\prime}+x_{2}^{\prime} \alpha_{2}^{\prime}+x_{3}^{\prime} \gamma_{3}^{\prime}},
\end{gathered}
$$

quando con $x_{1}^{\prime}, x_{2}^{\prime}, x_{3}^{\prime}$ si intendono le coordinate della retta $n^{\prime}$. Così i quattro piani $b_{1} a_{1}, b_{1} c_{1}, b_{1} m^{\prime}, b_{1} n^{\prime}$ corrispondono ai valori $0, \infty, \mu_{1}$ e $\mu_{2}$ del parametro $\mu$, epperò il loro rapporto anarmonico è dato dalla formula

$$
b_{3}\left(a_{1} c_{1} m^{\prime} n^{\prime}\right)=\mu_{1}: \mu_{2}=\frac{C^{\prime}}{A^{\prime}} \cdot \frac{x_{1}^{\prime} \alpha_{1}^{\prime}+x_{2}^{\prime} \alpha_{2}^{\prime}+x_{3}^{\prime} \alpha_{3}^{\prime}}{x_{1}^{\prime} \gamma_{1}^{\prime}+x_{2}^{\prime} \gamma_{2}^{\prime}+x_{3}^{\prime} \gamma_{3}^{\prime}} \text {. }
$$

I piani $b_{1}^{\prime} c_{1}^{\prime}$ e $b_{1}^{\prime} a_{1}^{\prime}$ della stella $\left(O^{\prime \prime}\right)$ hanno per equazioni

$$
x^{\prime \prime}{ }_{1}=0, \quad x^{\prime \prime}{ }_{3}=0 \text {, }
$$

e quindi l'equazione di un piano qualunque del fascio che ha per asse la retta $b_{1}^{\prime}$, ̀̀

$$
x^{\prime \prime}+\mu x^{\prime \prime}{ }_{3}=0 \text {. }
$$

I valori del parametro $\mu$, quando il piano del fascio passa per la retta data $m^{\prime \prime}$, o per la retta $n^{\prime \prime}$ corrispondente ad $n^{\prime}$, sono

$$
\mu=\mu^{\prime}=-\frac{m^{\prime \prime}{ }_{1}}{m^{\prime \prime}{ }_{3}}, \quad \mu=\mu^{\prime \prime}=-\frac{x^{\prime \prime}{ }_{1}}{x^{\prime \prime}{ }_{3}}
$$

quando con $x^{\prime \prime}{ }_{1}, x^{\prime \prime}{ }_{2}, x^{\prime \prime}{ }_{3}$ si intendano le coordinate della retta $n^{\prime \prime}$. Cosi $\mathrm{i}$ 
d'una superficie algebrica per mezzo di trasform. biraz. dello spazio.

quattro piani $b_{1}^{\prime} c_{1}^{\prime}, b_{1}^{\prime} a_{1}^{\prime}, b_{1}^{\prime} m^{\prime \prime}, b_{1}^{\prime} n^{\prime \prime}$ corrispondono ai valori $0, \infty, \mu^{\prime}, \mu^{\prime \prime}$ del parametro $\mu$, epperò il loro rapporto anarmonico è dato dalla formola

$$
b_{1}^{\prime}\left(c_{1}^{\prime} a_{1}^{\prime} m^{\prime \prime} n^{\prime \prime}\right)=\mu^{\prime}: \mu^{\prime \prime}=\frac{m_{1{ }_{1}} x_{3}^{\prime \prime}}{m_{3}^{\prime \prime} x_{1}^{\prime \prime}} .
$$

Ora in virtù della trasformazione quadratica fra le due stelle $\left(O^{\prime}\right),\left(O^{\prime \prime}\right)$, quest'ultimo rapporto anarmonico deve essere eguale al precedente, epperò si ha:

$$
\frac{C^{\prime}}{A^{\prime}} \cdot \frac{\alpha_{1}^{\prime} x_{1}^{\prime}+\alpha_{2}^{\prime} x_{2}^{\prime}+\alpha_{3}^{\prime} x_{3}^{\prime}}{\gamma_{1}^{\prime} x_{1}^{\prime}+\gamma_{2}^{\prime} x_{2}^{\prime}+\gamma^{\prime} x_{3}^{\prime}}=\frac{m^{\prime \prime}{ }_{1} x^{\prime \prime}{ }_{3}}{m_{3}^{\prime \prime} x^{\prime \prime}{ }_{1}} \text {. }
$$

Analogamente, esprimendo l'eguaglianza degli altri due rapporti anarmonici $c_{1}\left(a_{1} b_{1} m^{\prime} n^{\prime}\right)$ e $c^{\prime}{ }_{1}\left(b_{1}^{\prime} a_{1}^{\prime}{ }_{1} m^{\prime \prime} n^{\prime \prime}\right)$, si trova :

$$
\frac{B^{\prime}}{A^{\prime}} \cdot \frac{\alpha_{1}^{\prime} x_{1}^{\prime}+\alpha_{2}^{\prime} x_{2}^{\prime}+\alpha_{3}^{\prime} x_{3}^{\prime}}{\beta_{1}^{\prime} x_{1}^{\prime}+\beta_{2}^{\prime} x_{2}^{\prime}+\beta_{3}^{\prime} x_{3}^{\prime}}=\frac{m_{1}^{\prime \prime} x_{2}^{\prime \prime}}{m_{2}^{\prime \prime} x_{1}^{\prime \prime}} \text {. }
$$

Risolvendo rispetto alle $x^{\prime \prime}{ }_{1}, x^{\prime \prime}{ }_{2}, x^{\prime \prime}{ }_{3}$ il sistema formato da questa equazione e dalla precedente, e per brevità ponendo

ed inoltre

$$
m^{\prime \prime} A^{\prime}=A, \quad m_{2}^{\prime \prime} B^{\prime}=B, \quad m^{\prime \prime}{ }_{3} C^{\prime}=C,
$$

$$
\begin{gathered}
\alpha_{1}^{\prime} x_{1}^{\prime}+\alpha_{2}^{\prime} x_{2}^{\prime}+\alpha_{3}^{\prime} x_{3}^{\prime}=P^{\prime}, \quad \beta_{1}^{\prime} x_{4}^{\prime}+\beta_{2}^{\prime} x_{2}^{\prime}+\beta_{3}^{\prime} x_{3}^{\prime}=Q^{\prime}, \\
\gamma_{1}^{\prime} x_{1}^{\prime}+\gamma_{2}^{\prime} x_{2}^{\prime}+\gamma_{3}^{\prime} x_{3}^{\prime}=R^{\prime},
\end{gathered}
$$

si trova:

$$
\left.\begin{array}{l}
\rho^{\prime \prime} x_{1}^{\prime \prime}=A Q^{\prime} R^{\prime} \\
\rho^{\prime \prime} x_{2}^{\prime \prime}=B R^{\prime} P^{\prime} \\
\rho^{\prime \prime} x_{3}^{\prime \prime}=C P^{\prime} Q^{\prime},
\end{array}\right\}
$$

e queste formule danno le coordinate del raggio $n^{\prime \prime}$ della stella $\left(O^{\prime \prime}\right)$ corrispondente al raggio $n^{\prime}$ dato nella stella $\left(O^{\prime}\right)$.

Si risolva il medesimo sistema di equazioni rispetto alle $x_{1}^{\prime}, x_{2}^{\prime}, x_{3}^{\prime}$. Si ha intanto

$$
\begin{aligned}
& \alpha_{1}^{\prime} x_{1}^{\prime}+\alpha_{2}^{\prime} x_{2}^{\prime}+\alpha_{3}^{\prime} x_{3}^{\prime}=A x^{\prime \prime}{ }_{2} x^{\prime \prime}{ }_{3} \\
& \beta_{1}^{\prime} x_{1}^{\prime}+\beta_{2}^{\prime} x_{2}^{\prime}+\beta_{3}^{\prime} x_{3}^{\prime}=B x^{\prime \prime}{ }_{3} x^{\prime \prime}{ }_{1} \\
& \gamma_{1}^{\prime} x_{1}^{\prime}+\gamma_{2}^{\prime} x_{2}^{\prime}+y_{3}^{\prime} x_{3}^{\prime}=C x^{\prime \prime}{ }_{1} x^{\prime \prime}{ }_{2},
\end{aligned}
$$

donde, ricordando le note relazioni che passano fra i minori del determinante 
reciproco $H^{\prime}$ e gli elementi del primitivo ed inoltre ponendo

$B b_{1}^{\prime} x^{\prime \prime}{ }_{3}+C c^{\prime}{ }_{1} x^{\prime \prime}{ }_{2}=P^{\prime \prime}, \quad A a_{2} x^{\prime \prime}{ }_{2} x^{\prime \prime}{ }_{3}+B b_{2}^{\prime} x^{\prime \prime}{ }_{3} x^{\prime \prime}{ }_{1}+C c_{2}^{\prime} x^{\prime \prime}{ }_{1} x^{\prime \prime}{ }_{2}=Q^{\prime \prime}$,

si trova:

$$
-A a_{3} x_{2}^{\prime \prime} x_{3}^{\prime \prime}+B b_{3}^{\prime} x_{3}^{\prime \prime} x^{\prime \prime}{ }_{1}+C c_{3}^{\prime} x^{\prime \prime}{ }_{1} x^{\prime \prime}{ }_{2}=R^{\prime \prime} \text {, }
$$

$$
\begin{aligned}
& x_{1}^{\prime}=x^{\prime \prime}{ }_{1} P^{\prime \prime} \\
& x^{\prime}{ }_{2}=Q^{\prime \prime} \\
& x_{3}^{\prime}=R^{\prime \prime},
\end{aligned}
$$

e queste formule danno le coordinate del raggio $n^{\prime}$ della stella $\left(O^{\prime}\right)$ corrispondente al raggio $n^{\prime \prime}$ dato nella stella $\left(O^{\prime \prime}\right)$.

Ottenute le formule (8) e (9), come nel n. 1 si trovarono le (3) e (4), cosi qui si ricavano le seguenti

$$
\begin{aligned}
& x^{\prime \prime}{ }_{1}=A Q^{\prime} R^{\prime} p^{\prime} \\
& x^{\prime \prime}{ }_{2}=B R^{\prime} P^{\prime} p^{\prime} \\
& x^{\prime \prime}{ }_{3}=C P^{\prime} Q^{\prime} p^{\prime} \\
& x^{\prime \prime}{ }_{4}=\left(a^{\prime \prime}{ }_{1} A Q^{\prime} R^{\prime}+a^{\prime \prime}{ }_{2} B R^{\prime} P^{\prime}+a^{\prime \prime}{ }_{3} C P^{\prime} Q^{\prime}\right) x^{\prime}{ }_{4},
\end{aligned}
$$

e le loro inverse

$$
\begin{aligned}
& x_{1}^{\prime}=x_{1}^{\prime \prime} P^{\prime \prime} p^{\prime \prime} \\
& x_{2}^{\prime}=Q^{\prime \prime} p^{\prime \prime} \\
& x_{3}^{\prime}=R^{\prime \prime} p^{\prime \prime} \\
& x_{1}^{\prime}=\left(a_{1}^{\prime} x^{\prime \prime}{ }_{1} P^{\prime \prime}+a_{2}^{\prime} Q^{\prime \prime}+a_{3}^{\prime} R^{\prime \prime}\right) x_{1}^{\prime \prime} .
\end{aligned}
$$

Quelle formule servono a passare da un punto $P^{\prime}$ di $\Sigma^{\prime}$ al punto corrispondente $P^{\prime \prime}$ di $\Sigma^{\prime \prime}$ nella trasformazione cubica stabilita fra i due spazi $\Sigma^{\prime}$ e $\Sigma^{\prime \prime}$; queste servono al passaggio inverso.

14. In virtù delle due trasformazioni cubiche che hanno luogo l' una fra gli spazi $\Sigma$ e $\Sigma^{\prime}$ e l'altra fra $\Sigma^{\prime}$ e $\Sigma^{\prime \prime}$, fra il primo spazio e quest'ultimo nasce una terza trasformazione birazionale, di cui è facile trovare le formule.

Sia $P$ un punto dello spazio $\Sigma$. Ad esso corrisponde in $\Sigma^{\prime}$ un punto $P^{\prime}$, di cui le coordinate sono date dalle formule (6). Posti i valori di queste coordinate nelle $(10)$, si ottengono le formule seguenti

$$
\left.\begin{array}{l}
\rho^{\prime \prime} x^{\prime \prime}{ }_{1}=A Q R p \\
\rho^{\prime \prime} x^{\prime \prime}{ }_{2}=B R P p \\
\rho^{\prime \prime} x^{\prime \prime}{ }_{3}=C P Q p \\
\rho^{\prime \prime} x^{\prime \prime}{ }_{1}=\left(a^{\prime \prime} A Q R+a_{1}^{\prime \prime}{ }_{2} B R P+a^{\prime \prime}{ }_{3} C P Q\right) x_{4},
\end{array}\right\}
$$


nella quale ̀̀

$$
\begin{aligned}
& P=\alpha_{1}^{\prime} x_{2} x_{3}+\alpha_{2}^{\prime} x_{3} x_{1}+\alpha_{3}^{\prime} x_{1} x_{2}=\alpha_{1}^{\prime} x_{2} x_{3}+\pi x_{1} \\
& Q=\beta_{1}^{\prime} x_{2} x_{3}+\beta_{2}^{\prime} x_{3} x_{1}+\beta_{3}^{\prime} x_{1} x_{2}=\beta_{1}^{\prime} x_{2} x_{3}+c^{\prime} p x_{1} \\
& R=\gamma_{1}^{\prime} x_{2} x_{3}+\gamma_{2}^{\prime} x_{3} x_{1}+\gamma_{3}^{\prime} x_{1} x_{2}=\gamma_{1}^{\prime} x_{2} x_{3}-b^{\prime}{ }_{1} p x_{1},
\end{aligned}
$$

ed inoltre

$$
\pi=\alpha_{3}^{\prime} x_{2}+\alpha_{2}^{\prime} x_{3} \text {. }
$$

E queste formule servono a calcolare le coordinate del punto $P^{\prime \prime}$ di $\Sigma^{\prime \prime}$ corrispondente al punto $P$ dato in $\Sigma$.

Sia $P^{\prime \prime}$ un punto dello spazio $\Sigma^{\prime \prime}$. Ad esso corrisponde in $\Sigma^{\prime}$ un punto $P^{\prime}$, di cui le coordinate sono date dalle formule (11). Posti i valori di queste coordinate nelle (5), si ottengono le formule seguenti:

dove ̀̀

$$
\begin{aligned}
& \rho x_{1}=Q^{\prime \prime} R^{\prime \prime} p^{\prime \prime} \\
& \rho x_{2}=R^{\prime \prime} P^{\prime \prime} p^{\prime \prime} x^{\prime \prime} \\
& \rho x_{3}=P^{\prime \prime} Q^{\prime \prime} p^{\prime \prime} x^{\prime \prime}{ }_{1} \\
& \rho x_{4}=P^{\prime \prime} r^{\prime \prime} x_{1}^{\prime \prime} x^{\prime \prime}
\end{aligned}
$$

$$
r^{\prime \prime}=B \gamma_{1}^{\prime} x^{\prime \prime}{ }_{3}-C \beta_{1}^{\prime} x^{\prime \prime}{ }_{2} \text {. }
$$

E queste formule servono a calcolare le coordinate del punto $P$ di $\Sigma$ corrispondente al punto $P^{\prime \prime}$ dato in $\Sigma^{\prime \prime}$.

15. Ad un piano $U$ di $\Sigma$ che ha per equazione

$$
u_{1} x_{1}+u_{2} x_{2}+u_{3} x_{3}+u_{4} x_{1}=0,
$$

corrisponde in $\Sigma^{\prime \prime}$ una superficie $\varphi^{\prime \prime}$ di cui l'equazione è

$$
\left(u_{1} Q^{\prime \prime} R^{\prime \prime}+u_{2} R^{\prime \prime} P^{\prime \prime} x^{\prime \prime}{ }_{1}+u_{3} P^{\prime \prime} Q^{\prime \prime} x^{\prime \prime}{ }_{3}\right) p^{\prime \prime}+u_{4} P^{\prime \prime} r^{\prime \prime} x_{i}^{\prime \prime 2} x_{4}^{\prime \prime}=0 .
$$

Quindi la superficie $\varphi^{\prime \prime}$ è del $5 .^{\circ}$ ordine e possiede in $O^{\prime \prime}$ un punto quadruplo nel quale il cono tangente si scinde nei piani

$$
P^{\prime \prime}=0, \quad r^{\prime \prime}=0, \quad x^{\prime \prime}{ }_{1}=0,
$$

quest' ultimo contato due volte. Inoltre essa contiene la retta $s^{\prime \prime}$, le tre rette $h^{\prime \prime}, g^{\prime \prime}, l^{\prime \prime}$ nelle quali il piano $O^{\prime \prime} s^{\prime f}\left(p^{\prime \prime}=0\right)$ taglia i tre piani precedenti e lungo quest' ultima retta tocca lo stesso piano $O^{\prime \prime} s^{\prime \prime}$. Infine possiede come doppie le tre rette $a_{1}^{\prime}, b_{1}^{\prime}, c_{1}^{\prime}$ e come semplici le rette $a^{\prime \prime}, b^{\prime \prime}, c^{\prime \prime}$ corrispondenti alle rette $a^{\prime}, b^{\prime}, c^{\prime}$ di $\Sigma^{\prime}$. Le quali proprietà si dimostrano anche os- 
servando, che $\varphi^{\prime \prime}$ è la superficie di $\Sigma^{\prime \prime}$, che nella trasformazione fra $\Sigma^{\prime}$ e $\Sigma^{\prime \prime}$, corrisponde alla superficie $\varphi^{\prime}$ di $\Sigma^{\prime}$ corrispondente al piano dato $U$ nella trasformazione fra $\Sigma$ e $\Sigma^{\prime}$. Dunque:

I. "Ad un piano $U$ di $\Sigma$ corrisponde in $\Sigma$ " una superficie del "5. ordine $\varphi^{\circ}$, la quale possiede in $O^{\prime \prime}$ un determinato punto quadruplo, "contiene come semplici le rette $s^{\prime \prime}, a^{\prime \prime}, b^{\prime \prime}, c^{\prime \prime}, h^{\prime \prime}, g^{\prime \prime}, l^{\prime \prime}$, lungo l'ultima "delle quali tocea il piano $O^{\prime \prime} s^{\prime \prime}$ e come doppie le rette $a_{1}^{\prime}, b_{1}^{\prime}, c_{1}^{\prime}$."

Quindi gli elementi fondamentali dello spazio $\Sigma^{\prime \prime}$ sono il punto $O^{\prime \prime}$, le rette $a^{\prime \prime}, b^{\prime \prime}, c^{\prime \prime}, a_{1}^{\prime}, b_{1}^{\prime}, c_{1}^{\prime}, h^{\prime \prime}, g^{\prime \prime}, l^{\prime \prime}$, uscenti dal punto $O^{\prime \prime}$, la retta a quest'ultima infinitamente vicina sul piano $O^{\prime \prime} s^{\prime \prime}$ e la retta $s^{\prime \prime}$, alla quale si appoggiano ancora $h^{\prime \prime} g^{\prime \prime} l^{\prime \prime}$.

Ad un piano $V^{\prime \prime}$ di $\Sigma^{\prime \prime}$ condotto per il punto $O^{\prime \prime}$ e avente per equazione

$$
v^{\prime \prime}{ }_{1} x_{1}+v_{2}{ }_{2} x_{2}+v^{\prime \prime}{ }_{3} x_{3}=0 \text {, }
$$

corrisponde in $\Sigma$ il luogo di cui l'equazione è

$$
\left(v^{\prime \prime}{ }_{1} A Q R+v^{\prime \prime} B R P+v^{\prime \prime}{ }_{3} C^{\prime} P Q\right) p=0 \text {, }
$$

e che perciò si scinde nel piano fisso $O s$ e in un cono quadrico $\Gamma_{0}$. Questo è il cono che nella trasformazione quadratica fra le due stelle $(0)$ ed $\left(O^{\prime}\right)$, corrisponde al cono $\Gamma_{1}$ di quest' ultima, corrispondente al piano dato $V^{\prime \prime}$, nell'altra trasformazione quadratica fra le due stelle $\left(O^{\prime}\right)$ ed $\left(O^{\prime \prime}\right)$. Quindi esso possiede come doppie le tre rette $a, b, c$ e come semplici le tre rette $a_{0}$, $b_{0}, c_{0}$ corrispondenti in $\Sigma$ alle tre rette $a_{1}, b_{1}, c_{1}$ di $\Sigma^{\prime}$, la prima delle quali, $a_{0}$, è infinitamente vicina ad $a$, sopra il piano $O s$, la retta $a_{i}$ essendo l'intersezione del piano $b c$ col piano $r$. Del resto queste proprietà del cono $\Gamma_{0}$ possono essere dedotte facilmente anche dalla sua equazione, dalla quale in particolare si deduce che dei due piani ad esso tangenti lungo la retta $a$, uno ha per equazione $p=0$ (epperò è il piano $O s$ ) e l'altro:

$$
v_{1}{ }_{1} A b_{1}^{\prime} c_{1} p+\left(v^{\prime \prime}{ }_{2} B b^{\prime}{ }_{1}-v^{\prime \prime}{ }_{2} C c^{\prime}{ }_{1}\right) \pi=0 \text {. }
$$

Dunque:

II. "Ad un piano $V^{\prime \prime}$ di $\Sigma^{\prime \prime}$ condotto per il punto $O^{\prime \prime}$ corrisponde " in $\mathbf{\Sigma}$ un cono del $4 .^{\circ}$ ordine $\Gamma_{0}$, il quale possiede come semplici le rette « $b_{0}$ e $c_{0}$ e come doppie le rette $a, b, c$, lungo la prima delle quali ha come "uno dei piani tangenti il piano fisso $O s$."

In particolare, al piano $O^{\prime \prime} s^{\prime \prime}$, di cui l'equazione è $p^{\prime \prime}=0$, corrisponde il cono $C_{0}$, che ha per equazione

$$
Q_{0}=a^{\prime \prime} A Q R+a_{1}^{\prime \prime} B R P+a^{\prime \prime}{ }_{3} C P Q=0,
$$


d' una superficie algebrica per mezzo di trasform. biraz. dello spazio. 91

e che lungo la generatrice doppia $a$ è toccato dal piano $O s$ e dal piano she ha per equazione

$$
a^{\prime \prime}{ }_{1} A b^{\prime}{ }_{1} c^{\prime}{ }_{1} p+\left(a^{\prime \prime}{ }_{2} B b_{1}^{\prime}-a^{\prime \prime}{ }_{3} C c^{\prime}{ }_{1}\right) \pi=0 .
$$

Il cono $C_{0}$ viene tagliato dal piano $O s$, oltre la retta $a$ contata tre volte, in un' altra retta $g$.

Ad un piano $U^{\prime \prime}$ di $\Sigma^{\prime \prime}$ avente per equazione

$$
u^{\prime \prime} x_{1}{ }_{1}+u^{\prime \prime}{ }_{2} x_{2}+u^{\prime \prime}{ }_{3} x_{3}+u^{\prime \prime}{ }_{4} x_{4}=0
$$

corrisponde in $\Sigma$ una superficie $\varphi_{0}$, di cui l'equazione è

$$
\left(u^{\prime \prime}{ }_{1} A Q R+u^{\prime \prime}{ }_{2} B R P+u^{\prime \prime} C P Q\right) p+u^{\prime \prime}{ }_{4} Q_{0} x_{4}=0 .
$$

Quindi la superficie $\varphi_{0}$ è del $5 .^{\circ}$ ordine e possiede in $O$ un punto quadruplo, nel quale il cono tangente è il cono fisso $C_{0}$. Ordinando l'equazione precedente rispetto alla variabile $x_{1}$, si trova che la più alta potenza di questa che figuri nell'equazione stessa, è il quadrato. Quindi si conclude intanto che la superficie $\varphi_{0}$ è dotata in $A$ di un punto triplo. Inoltre, se si pone eguale a zero, il coefficiente di $x_{1}^{2}$, si ottiene un'equazione che si scinde in due: una è $p=0$, e l'altra è la seguente:

$$
\begin{gathered}
{\left[u_{1}^{\prime \prime} A b_{1}^{\prime} c_{1}^{\prime} p+\left(u_{2}^{\prime \prime} B b_{1}^{\prime}-u_{{ }_{3}} C c_{1}\right) \pi\right] p} \\
+u_{4}^{\prime \prime}\left[a_{1}^{\prime \prime}{ }_{1} A b_{1}^{\prime} c_{1}^{\prime} p+\left(a_{2}^{\prime \prime} B b_{1}^{\prime}-a_{3}^{\prime \prime} C c_{1}^{\prime}\right) \pi\right] x_{4}=0 .
\end{gathered}
$$

Questa rappresenta un cono quadrico contenente le rette $s$ ed $a$ e toccato lungo quest' ultima dal medesimo piano $k$ tangente lungo la stessa generatrice al cono $C_{0}$. Dunque il cono tangente nel punto $A$ alla superficie $\varphi_{0}$ si scinde nel piano $O s$ e nell'anzidetto cono quadrico. Inoltre questa superficie possiede come semplici le rette $s, g, b_{0}, c_{0}$ e come doppie le rette $a, b, c$, lungo la prima delle quali è toccata dai medesimi due piani che toccano il cono $C_{0}$, il che puo anche essere dimostrato formando l'equazione complessiva dei piani stessi. Ma la falda della superficie $\varphi_{0}$ tangente lungo la retta $a$ al piano $O s$ ha con la falda del cono $C_{0}$ tangente lungo la medesima retta allo stesso piano un contatto d'ordine superiore.

Per vedere quale sia questo contatto, si operi una sezione $f_{0}$ nella superficie $\varphi_{0}$ col piano

$$
\lambda_{1} x_{1}+\lambda_{2} x_{2}+\lambda_{3} x_{3}+x_{4}=0 ;
$$

e per conoscere poi le proprietà di questa sezione facciasi una trasformazione 
di coordinate ponendo

$$
\begin{aligned}
x_{1} & =y_{1} \\
x_{3} & =y_{2} \\
x_{3} & =y_{3} \\
\lambda_{1} x_{1}+\lambda_{2} x_{2}+\lambda_{3} x_{3}+x_{1} & =y_{4},
\end{aligned}
$$

ossia$$
x_{1}=y_{1}
$$$$
x_{2}=y_{2}
$$$$
x_{3}=y_{3}
$$$$
x_{1}=y_{4}-\left(\lambda_{1} y_{1}+l_{1}\right) \text {, }
$$

dove è

$$
l_{1}=\lambda_{2} y_{2}+\dot{\lambda}_{3} y_{3} .
$$

L' equazione della superficie $\varphi_{0}$ si cambia in un' altra, nella quale ponendo $y_{4}=0$, si ottiene

dove ̀̀

$$
\left.\begin{array}{rl} 
& \left(L_{a} \lambda_{1} y_{1} u^{\prime \prime}{ }_{4}+L_{a} l_{1} u^{\prime \prime}{ }_{4}-L_{u} p_{1}\right) p_{1} y_{1}{ }^{2} \\
+ & \left(M_{a} \lambda_{3} y_{1} u^{\prime \prime}{ }_{4}+M_{a b} l_{1} u^{\prime \prime}{ }_{4}-M_{u} p_{1}\right) y_{1} y_{2} y_{3} \\
+ & \left(N_{a} \lambda_{1} y_{1} u^{\prime \prime}{ }_{4}+N_{a} l_{1} u^{\prime \prime}{ }_{4}-N_{u} p_{1}\right) y_{2}{ }^{2} y_{3}{ }^{2}=0,
\end{array}\right\}
$$

$L_{u}=u^{\prime \prime}{ }_{1} A b^{\prime}{ }_{1} c^{\prime}{ }_{1} p_{1}+u^{\prime \prime}{ }_{2} B b_{1}^{\prime} \pi_{1}-u^{\prime \prime}{ }_{3} C c^{\prime}{ }_{1} \pi_{1}$

$M_{u}=u^{\prime \prime}{ }_{1} A\left(\beta_{1}^{\prime} b_{1}^{\prime}-\gamma^{\prime} c_{1}^{\prime}{ }_{1}\right) p_{1}+u^{\prime \prime}{ }_{2} B\left(\alpha^{\prime}{ }_{1} b_{1}{ }_{1} p_{1}-\gamma^{\prime}{ }_{1} \pi_{1}\right)-u^{\prime \prime}{ }_{3} C\left(\alpha^{\prime}{ }_{1} c^{\prime}{ }_{1} p_{1}+\beta^{\prime}{ }_{1} \pi_{1}\right)$

$N_{u}=-\left(u_{1}^{\prime \prime} A \beta_{1}^{\prime} \gamma_{1}^{\prime}+u_{2}^{\prime \prime} B \gamma_{1}^{\prime} \alpha_{1}^{\prime}+u_{3}^{\prime \prime} C \alpha_{1}^{\prime} \beta_{1}^{\prime}\right)$,

e $I_{a}, M_{a}, N_{a}$ rappresentano le medesime espressioni precedenti, nelle quali si pongano $a_{1}^{\prime \prime}, a_{2}^{\prime \prime}, a_{3}^{\prime \prime}$ in luogo di $u_{1}^{\prime \prime}, u_{2}^{\prime \prime}, u_{3}^{\prime \prime}$ rispettivamente, e infino $p_{1}$ e $\pi_{1}$ ciò che diventano $p$ e $\pi$ quando in queste funzioni si sostituiscono $y_{2}$ e $y_{3}$ ad $x_{2}$ e $x_{3}$. L'equazione (14) rappresenta la sezione fatta nella superficie $\varphi_{0}$ dal piano dato, riferita alle nuove coordinate $y_{1}, y_{2}, y_{3}, y_{4}$. La sua forma mostra che questa sezione possiede un punto doppio nel punto A in cui il suo pjano incontra la retta $a$ e che in questo punto le rette tangenti hanno per equazioni

$$
L_{a}=0, \quad p_{1}=0,
$$

il che è in accordo con le proprietà già trovate per la superficie $\varphi_{0}$. 
d' 'nna superficie algebrica per mezzo di trasform. biraz. dello spazio. 93

Ora sul piano $y_{4}=0$ facciasi una trasformazione quadratica ponendo

$$
y_{1}=y_{2}^{\prime} y_{3}^{\prime}, \quad y_{2}=y_{3}^{\prime} y_{1}^{\prime}, \quad y_{3}=y_{1}^{\prime} y_{2}^{\prime} .
$$

In virtù di questa trasformazione, la curva $f_{0}$ si cambia in un'altra $f^{\prime}{ }_{0}$, di cui l'equazione è

$$
\begin{aligned}
& \left(L_{a}^{\prime} \lambda_{1} y_{2}^{\prime} y_{3}^{\prime} u_{4}^{\prime \prime}+L_{a}^{\prime} l_{1}^{\prime} y_{1}^{\prime} u_{1{ }_{4}}-L_{u}^{\prime} p_{1}^{\prime} y_{1}^{\prime}{ }_{1}\right) p_{1}^{\prime_{1}} \\
& +\left(M_{a}^{\prime} \lambda_{1} y_{2}^{\prime} y_{3}^{\prime} u^{\prime \prime}{ }_{4}+M_{a}^{\prime} l_{1}^{\prime} y^{\prime}{ }_{1} u^{\prime \prime}{ }_{4}-M_{u}^{\prime} p_{1}^{\prime} y_{1}^{\prime}\right) y_{1}^{\prime}
\end{aligned}
$$

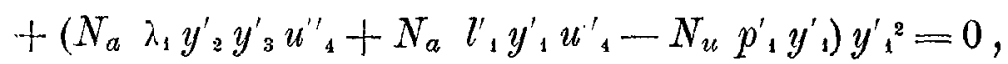

dove $L_{u}^{\prime}, M^{\prime}{ }_{u}, L_{a}^{\prime}, M_{a}^{\prime}, p^{\prime}$, rappresentano i risultati delle sostituzioni di $y_{3}$ e $y_{z}$ ad $x_{2}$ e $x_{3}$ nelle funzioni $L_{u}, M_{u}, L_{a}, M_{a}, p_{1}$. Quindi le rette

$$
L_{t}^{\prime}=0, \quad p_{1}^{\prime}=0,
$$

corrispondono nella trasformazione quadratica alle tangenti in alla curva $f_{0}$; epperò la curva $f^{\prime}$ o incontra la retta fondamentale $y_{1}^{\prime}=0$, nei punti in cui questa è tagliata dalle due rette anzidette. La tangente ad $f^{\prime}{ }_{0}$ nel primo di questi due punti ha per equazione

$$
\left(M^{\prime \prime}{ }_{a} \lambda_{1} \alpha \beta u^{\prime \prime}{ }_{4}-L^{\prime \prime}{ }_{u} p^{\prime 2}\right) y_{1}^{\prime}+\lambda_{1} \alpha \beta u^{\prime \prime}{ }_{4} p^{\prime \prime}{ }_{1} . L_{a}^{\prime}=0,
$$

dove a e $\beta$ sono le coordinate del punto di contatto, $M^{\prime \prime}{ }_{a}, L^{\prime \prime}{ }_{u}, p^{\prime \prime}, \mathrm{i}$ valori che prendono le funzioni $M^{\prime}{ }_{a}, L_{u}^{\prime}, p_{1}^{\prime}$ quando queste coordinate si so stituiscono alla variabile $y_{2}^{\prime}$ e $y_{3}^{\prime}$. La conica che nella trasformazione quadratica corrisponde a questa tangente, oscula nel punto $A$ il ramo della curva $f_{0}$ che ivi tocca la retta $L_{a}$. Ma quella tangente è variabile con la superficie $\varphi_{0}$, perchè nella sua equazione entrano le coordinate $u^{\prime \prime}{ }_{1}, u^{\prime \prime}{ }_{2}, u^{\prime \prime}{ }_{3}$, $u^{\prime \prime}{ }_{4}$ del piano $U^{\prime \prime}$ di $\Sigma^{\prime \prime}$, cui corrisponde $\varphi_{0}$; dunque anche questa conica oseulatrice varia col variare della superficie $\varphi_{0}$. Quindi si conclude che Je falde di due superficie qualsivogliano $\varphi_{0}$ tangenti lungo la retta $a$ al medesimo piano $k$ hanno in comune, oltre $a$, una sola retta a questa infinitamente vicina; epperò ciascuna di esse ha anche in comune, oltre $a$, una sola retta a questa infinitamente vicina con la falda del cono $C_{0}$ tangente lungo $a$ al piano $k$.

Ciò premesso, si noti che il cono del $4 .^{\circ}$ ordine $C_{0}$ tangente alla superficie del $5 .^{\circ}$ ordine $\varphi_{0}$ nel punto quadruplo $O$, taglia questa in 20 rette uscenti da $O$. Il piano $U^{\prime \prime}$ di $\Sigma^{\prime \prime}$, cui corrisponde in $\Sigma$ la superficie $\varphi_{0}$, incontra la retta fondamentale $s^{\prime \prime}$ in un punto, al quale corrisponde in $\Sigma(4, \mathrm{~V}$ e $7, \mathrm{IV})$ una retta $x$ che appartiene tanto a $\varphi_{0}$ quanto a $C_{0}$. Queste due superficie 
hanno inoltre in comune le rette $g, b_{0}, c_{0}$ e le rette $a, b, c$; quelle sono semplici per ciascuna di esse, e queste sono doppie, e i piani tangenti alla superficie $\rho_{0}$ in un punto della retta $b, 0 c$, sono, in generale, distinti da quelli che lungo la medesima retta toccano il cono $C_{0}$. Quindi delle 20 rette anzidette $1+1+1+1+2^{2}+2^{2}=12$ coincidono rispettivamente con le rette $x, g, b_{0}, c_{0}, b, c$; epperò le rimanenti 8 vengono assorbite dalla retta $a$. Ma per la proprietà che questa è doppia per $\varphi_{j}$ e per $C_{0}$, e che le falde di queste due superficie tangenti lungo $a$ al medesimo piano $k$ hanno in comune, fuori di $a$, una sola retta infinitamente vicina ad $a$, nella stessa retta $a$ vengono soltanto a cadere 5 delle 20 rette comuni a $\varphi_{0}$ e a $C_{0}$. Dunque se invece ve ne cadono 8 , vuol dire che le falde di queste due superficie tangenti lungo $a$ al piano $O s$ hanno in comune, oltre $a$, tre rette infinitamente vicine ad $a$.

Un piano condotto ad arbitrio per $A$ taglia le superficie $\varphi_{0}$ e $C_{0}$ secondo dne curve delle quali una possiede in $A$ un punto triplo e l'altra un punto doppio, e queste curve hanno in $A$ una sola tangente comune che è la retta secondo la quale il piano considerato incontra il piano $0 \mathrm{~s}$. Quindi dei punti comuni alle curve medesime $2.3+1=7$ cadono, per queste proprietà, nel punto $A$. Ma esse si tagliano, fuori di $A$, in altri 12 punti soltanto, i quali cadono nei punti in cui il loro piano incontra le rette $x, g, b_{0}, c_{0}, b, c$ comuni alle due superficie $\varphi_{0}$ e $C_{0}$. Dunque nel punto $A$ deve ancora cadere l'ulteriore loro punto d'incontro, il che porta a concludere che $\mathrm{i}$ rami di dette curve tangenti in $A$ al piano $O s$, hanno in comune, oltre $A$, due punti infinitamente vicini ad $A$. Così si vede che le falde delle due superficie $\varphi_{0}$ e $C_{0}$ tangenti in $A$ al piano $O s$, si osculano.

Riepilogando si ha dunque:

III. "Ad un piano qualunque $U^{\prime \prime}$ di $\Sigma^{\prime \prime}$ corrisponde in $\Sigma$ una su"perficie del $5.0^{\circ}$ ordine $\varphi_{0}$, la quale possiede in $O$ un punto quadruplo, in " cui il cono tangente è il cono fisso $C_{0}$, e in $A$ un punto triplo, in cui il " cono tangente si scinde nel piano fisso $O s$ e in un cono quadrico conte" nente le rette $s$ ed $a$. Inoltre essa possiede come semplici le rette $s, g$, " $b_{0}, c_{0}$ e come doppie le rette $a, b, c$, lungo la prima delle quali è toccata "da due piani tangenti fissi, $O s$ e $k$, che sono quelli stessi che lungo $a$ toc" cano il cono $C_{0}$, e di cui uno, $k$, tocea ancora l'anzidetto cono quadrico. "Infine la falda della superficie $\varphi_{0}$ tangente lungo $a$, al piano $O s$ ha in co"mune, oltre $a$, con la falda del cono $C_{0}$ tangente lungo la medesima retta " allo stesso piano, tre rette infinitamente vicine ad $a$, e le medesime falde " nel punto $A$ si osculano soltanto." 
d'una superficie algebrica per mezzo di trasform. biraz, dello spazio. 95

Una retta qualunque $R^{\prime \prime}$ di $\Sigma^{\prime \prime}$ può sempre essere considerata come l'intersezione del piano $V^{\prime \prime}$ che la proietta dal punto $O^{\prime \prime}$ con un altro piano $U^{\prime \prime}$ condotto ad arbitrio per essa. A quel piano corrisponde in $\Sigma$ (II) un cono $\Gamma_{0}$; a questo (III), una superficie $\varphi_{0}$. Quindi alla retta data corrisponde la curva d'intersezione variabile $S_{0}$ di queste due superficie. Le proprietà di questa curva si trovano ripetendo ragionamenti del tutto analoghi a quelli seguiti per dimostrare il teorema IV del n. ${ }^{\circ} 3$; e cos̀ si ottiene :

IV. "Ad una retta $R^{\prime}$ di $\Sigma^{\prime \prime}$ corrisponde in $\Sigma$ una curva del $5 .^{\circ}$ or" dine $S_{0}$, la quale possiede in $A$ un punto doppio, in cui una delle tangenti " è la retta $s$, passa semplicemente per il punto $O$ e tocea ivi il cono $C_{0}$, "si appoggia in un altro punto, fuori di $A$, alla retta $s$, e, fuori di $O$, in " un punto a ciascuna delle rette $b_{0} \mathrm{e} c_{0}, \mathrm{e}$ in due punti a ciascuna delle " rette $b$ e $c$."

Si può pervenire al medesimo risultato determinando prima la cubica $S_{1}$ di $\Sigma^{\prime}$ corrispondente $\left(3\right.$, IV) alla retta $R^{\prime \prime}$ di $\Sigma^{\prime \prime}$, e poi cercando, con l'aiuto di teoremi stabiliti nel $\S 2$, la curva che in $\Sigma$ corrisponde ad $S_{4}$.

16. Al piano $O s$ di $\Sigma$ corrisponde in $\Sigma^{\prime \prime}$ il piano $r^{\prime \prime}$ di cui l'equazione è $r^{\prime \prime}=0$; e al piano $O^{\prime \prime} s^{\prime \prime}$ di $\Sigma^{\prime \prime}$ corrisponde in $\Sigma$ il cono $C_{0}$. Quindi alla retta $g^{\prime \prime}$, intersezione dei due piani $r^{\prime \prime}$ ed $O^{\prime \prime} s^{\prime \prime}$, corrisponde la retta $g$, nella quale il piano $O s$, incontra, fuori di $a$, il cono $C_{0}$. Di qui segue:

I. "Ad un piano di $\Sigma^{\prime \prime}$ condotto per la retta $g^{\prime \prime}$, corrisponde in $\Sigma$ un u cono $\Gamma_{g}$ che gode di tutte le proprieta possedute da un cono $\Gamma_{0}$ e che inoltre "passa per lia retta g."

Ora cercando l'intersezione di questo cono con una superficie $\varphi_{0}$, si trova facilmente :

II. "Ad una retta di $\Sigma^{\prime \prime}$ appoggiata alla retta $g$ " corrisponde in $\Sigma$ " una curva del $4 .^{\circ}$ ordine, la quale possiede in $A$ un punto doppio, in cui " una delle tangenti è la retta $s$, si appoggia in un punto variabile a cia" scuna delle rette $g, b_{0}$ e $c_{0}$ e in due punti parimenti variabili a ciascuna "delle rette $b$ e $c$."

La retta $h^{\prime \prime}$ è l'intersezione dei due piani che hanno per equazioni

$$
p^{\prime \prime}=a^{\prime \prime}{ }_{1} x_{1}^{\prime \prime}+a^{\prime \prime}{ }_{2} x^{\prime \prime}{ }_{2}+a^{\prime \prime}{ }_{3} x^{\prime \prime}{ }_{3}=0, \quad P^{\prime \prime}=B b_{1}^{\prime} x^{\prime \prime}{ }_{3}+C c^{\prime} x^{\prime \prime}{ }_{2}=0 \text {. }
$$

Quindi l'equazione di un piano qualunque condotto per la retta stessa è

$$
\lambda a_{1}^{\prime \prime} x_{1}^{\prime \prime}+\left(\lambda a_{2}^{\prime \prime}+C c_{1}^{\prime}\right) x^{\prime \prime}{ }_{2}+\left(\lambda a_{3}{ }_{3}+B b_{1}^{\prime}\right) x^{\prime \prime}{ }_{3}=0 .
$$

A questo piano corrisponde in $\Sigma\left(15\right.$, II) un cono $\Gamma_{h}$ il quale possiede la 
retta a come generatrice doppia e dei due piani ad esso tang'enti lungo questa uno è $O s$ e l'altro, secondo la (14') ha per equazione

$$
\lambda a^{\prime \prime}{ }_{1} A b_{1}^{\prime} c_{1}{ }_{1} p+\left[\left(\lambda a^{\prime \prime}{ }_{2}+C c^{\prime}{ }_{1}\right) B b_{1}^{\prime}-\left(\lambda a^{\prime \prime}{ }_{3}+B b^{\prime}{ }_{1}\right) C c^{\prime}{ }_{1}\right] \pi=0
$$

ossia

$$
a^{\prime \prime}{ }_{1} A b^{\prime}{ }_{1} c_{1}^{\prime} p+\left(a^{\prime \prime}{ }_{2} B b^{\prime}{ }_{1}-a^{\prime \prime}{ }_{3} C c^{\prime}{ }_{1}\right) \pi=0 \text {, }
$$

che è appunto l'equazione del piano $k$. Dunque:

III. "Ad un piano di $\Sigma$ " condotto per la retta $h$ " corrisponde in $\Sigma$ " un cono $\Gamma_{h}$, che gode di tutte le proprietà possedute da un cono $\Gamma_{0}$ e che "inoltre è toccato lungo la retta $a$ non solo dal piano $O s$, ma anche dal "piano k."

Cercando l'intersezione di questo cono con una superficie $\varphi_{0}$, si trova:

IV. "Ad una retta di $\Sigma$ " appoggiata alla retta $h^{\prime \prime}$ corrisponde in $\Sigma$ " una curva del $4 .^{\circ}$ ordine, la quale passa per $A$ e tocca ivi la retta $s$, si " appoggia a questa retta in un altro punto, oltre $A$, in un punto, fuori di $A$ " e di $O$, alla retta $a$ e in questo tocca il piano $k$, e, fuori di 0 , in un punto " a ciascuna delle rette $b_{0}$ e $c_{0}$ e in due punti a ciascuna delle rette $b$ e $c$."

La retta $l^{\prime}$ è l'intersezione dei due piani $O^{\prime \prime} s^{\prime}$ e $b_{1}^{\prime} c_{1}{ }_{1}$ di cui le equazioni sono $p^{\prime \prime}=0$ e $x^{\prime \prime}{ }_{1}=0$. Quindi un piano qualunque che passi per $l^{\prime \prime}$, ha come equazione

$$
\lambda x_{1}^{\prime \prime}+a_{2}^{\prime \prime} x_{2}^{\prime \prime}+a_{3}^{\prime \prime} x_{3}^{\prime \prime}=0 .
$$

A questo piano corrisponde in $\Sigma$ un cono $\Gamma_{l}$, il quale possiede la retta $a$ come generatrice doppia, e dei due piani ad esso tangenti lungo questa uno è $O s$ è l'altro secondo la $\left(14^{\prime}\right)$ ba per equazione

$$
\lambda A b^{\prime}{ }_{1} c_{1}{ }_{1} p+\left(a^{\prime \prime}{ }_{2} B b^{\prime}{ }_{1}-a^{\prime \prime}{ }_{3} C c^{\prime}{ }_{1}\right) \pi=0 \text {, }
$$

epperò è distinto dal piano $k$. Ora l'anzidetto cono $\Gamma_{l}$ ha in comune col cono $C_{0}$, fuori di $a$, soltanto le due rette $b_{0}$ e $c_{0}$ e le due rette $b$ e $c$; e poichè quelle sono semplici e queste doppie per ciascun cono e di più i piani tangenti lungo esse ai due coni sono fra loro distinti, così le rette medesime assorbono 10 delle rette comuni ai coni $\Gamma_{l}$ e $C_{\lrcorner}$. Quindi in $a$ cadono le rimanenti 6 rette d'intersezione; e ciò, per la proprietà dianzi dimostrata, che i coni $\Gamma_{l}$ e $C_{0}$ hanno in comune lungo a solo il piano tangente $O s$, porta necessariamente a concludere che le falde dei coni medesimi che toccano questo piano, si osculano lungo a. Dunque:

V. "Ad un punto di $\Sigma^{\prime \prime}$ condotto per la retta $l$, corrisponde in $\Sigma$ "un cono $\Gamma_{l}$, che gode di tutte le proprietà possedute da un cono $\Gamma_{0}$ o che 
d' una superficie algebrica per mezzo di trasform. biraz. dello spazio. 97

" inoltre oscula con una falda lungo la retta $a$ la falda del cono $C_{0}$ tangente " lungo la medesima retta al piano $O s$."

Sia $H^{\prime}$ una quadrica di $\Sigma^{\prime}$ la quale contenga la retta $s^{\prime}\left(p^{\prime}=0, x_{4}^{\prime}=0\right)$, passi per il punto $O$ e tocchi ivi la retta $a_{1}\left(r^{\prime}=0, x^{\prime}=0\right)$. La sua equazione è della forma:

$$
\left(m_{1} x_{1}^{\prime}+m_{2} x_{2}^{\prime}+m_{3} x_{3}^{\prime}\right) p^{\prime}+\left(\lambda x_{1}^{\prime}+r^{\prime}\right) x_{4}^{\prime}=0,
$$

dove $m_{1}, m_{2}, m_{3}$ e $\lambda$ sono costanti date. A questa quadrica corrisponde in $\Sigma$ una superficie $H$, di cui l'equazione, in virtù delle formule $(6)$, è :

$$
\left(m_{3} x_{2}+m_{2} x_{3}+x_{4}\right) p x_{1}+\left(m_{1} p+\lambda x_{4}\right) x_{2} x_{3}=0 \text {. }
$$

Quindi la superficie $H$ è del $3 .^{\circ}$ ordine, contiene le rette $s, a, b, c$, possiede in $A$ un punto doppio biplanare, in $O$ un punto doppio conico e lungo la retta $a$ viene toccata dal piano fisso $O s$.

Se

$$
\left(n_{1} x_{1}^{\prime}+n_{2} x_{2}^{\prime}+n_{3} x_{3}^{\prime}\right) p^{\prime}+\left(\mu x_{1}^{\prime}+r^{\prime}\right) x_{4}^{\prime}=0,
$$

è l' equazione di una seconda quadrica $K^{\prime}$ di $\Sigma$ ', la quale passi ancora per la retta $s^{\prime}$, per il punto $O^{\prime}$ e tocchi ivi la retta $a_{1}$, l'equazione della superficie $K$ corrispondente in $\Sigma$ è

$$
\left(n_{3} x_{2}+n_{2} x_{3}+x_{4}\right) p x_{1}+\left(n_{1} p+\mu x_{4}\right) x_{2} x_{3}=0 .
$$

Le due quadriche $H^{\prime}$ e $K^{\prime}$ si tagliano oltre la retta $s^{\prime}$, secondo una cubica gobba, la quale ha questa retta come corda ed inoltre passa per il punto $O^{\prime}$ e tocea ivi la retta $a_{1}$. A questa cubica di $\Sigma^{\prime}$ corrisponde in $\Sigma$ la curva d'intersezione variabile delle due superficie $H$ e $K$, la quale curva è del $4 .^{\circ}$ ordine, perchè queste due superficie hanno in comune le 4 rette $s, a, b, c$, e lungo la retta $a$ sono toccate dallo stesso piano tangente $O s$. Ora si ha in generale, come è facile dimostrare, che se

$$
H=A_{1} p x_{1}+A_{3}=0, \quad K=B_{1} p x_{1}+B_{3}=0,
$$

dore $A_{1}, A_{3}, B_{1}, B_{3}$ rappresentino funzioni omogenee delle coordinate $x_{2}, x_{3}, x_{4}$ dei gradi indicati dai loro indici, sono le equazioni di due superficie di $3 .^{\circ}$ ordine, la loro curva d'intersezione possiede in $A$ un punto multiplo secondo 5 e le tangenti in questo punto sono la retta d'intersezione dei due piani $A_{1}=0, B_{1}=0$ e le 4 rette secondo cui il piano $p=0$ taglia il cono che ha per equazione:

$$
A_{1} B_{3}-A_{3} B_{4}=0 .
$$

Annali di Matematica, tomo XXV. 
Nel caso attuale è

$$
\begin{array}{ll}
A_{1}=m_{3} x_{2}+m_{2} x_{3}+x_{4}, & A_{3}=\left(m_{1} p+\lambda x_{4}\right) x_{2} x_{3}, \\
B_{1}=n_{3} x_{2}+n_{2} x_{3}+x_{4}, & B_{3}=\left(n_{1} p+\mu x_{4}\right) x_{2} x_{3} .
\end{array}
$$

Quindi come equazione dell'anzidetto cono si trova

$\left[\left(m_{3} x_{2}+m_{2} x_{3}+x_{4}\right)\left(n_{1} p+\mu x_{4}\right)-\left(n_{3} x_{2}+n_{2} x_{3}+x_{4}\right)\left(m_{1} p+\lambda x_{4}\right)\right] x_{2} x_{3}=0$, epperò il cono stesso si scinde nei due piani $x_{2}=0, x_{3}=0$ e in un cono quadrico. Il piano $p=0$ taglia ciascuno di quei due piani nella retta $a$, e questo cono nella retta $s$ e in un'altra retta, che è l'intersezione dello stesso piano $p=0$ con il piano che ba per equazione

$$
\left(m_{3} \mu-n_{3} \lambda\right) x_{z}+\left(m_{2} \mu-n_{3} \lambda\right) x_{3}+(\mu-\lambda) x_{4}=0,
$$

e che perciò è distinta tanto da $s$ quanto da $a$. Nel caso in esame adunque le tangenti nel punto $A$ alla intersezione delle due superficie date $H$ e $K$ sono: la retta a contata due volte, la retta $s$, una retta del piano $O s$ distinta da $a$ e da $s$ e una retta situata fuori di questo piano. Ma la retta $a$ contata due volte e la retta $s$ fanno parte di quella intersezione; dunque la rimanente intersezione, che come si è già osservato è una curva del $4 .^{2}$ ordine, possiede in $A$ un punto doppio e le sue tangenti in questo punto sono le due rimanenti delle cinque tangenti anzidette. Inoltre la medesima curva si appoggia in un punto, oltre $A$, alla retta $s$, e in due punti variabili a ciascuna delle rette $b$ e $c$, il che si può vedere facilmente cercando il numero dei punti d'intersezione di essa curva con un piano condotto per la retta $s, 0 b, o c$, situati fuori della retta stessa.

Ad una retta di $\Sigma^{\prime \prime}$ appoggiata alla retta $l^{\prime \prime}$ corrisponde in $\Sigma^{\prime}(5$, VIII) una cubica gobba $S_{1}$, la quale passa per il punto $O^{\prime}$, tocca ivi la retta $a_{1}$ ed oscula il piano di $\Sigma^{\prime}$ corrispondente al piano $a_{1}^{\prime} l^{\prime \prime}$ di $z^{\prime \prime}$, si appoggia in due punti alla retta $s^{\prime} \mathrm{e}$ in un punto a ciascuna delle due rette $b_{1}$ e $c_{1}$. Questa cubica appartiene al sistema delle cubiche di $\Sigma$ le cui curve corrispondenti dello spazio $\Sigma$ sono state ora studiate. Quindi tenendo presenti $i$ risultati ottenuti, si conclude:

VI. "Ad una retta di $\Sigma^{\prime \prime}$ appoggiata alla retta $l$ " corrisponde in $\Sigma$ * una curva del $4 .^{\circ}$ ordine, la quale possiede in $A$ un punto doppio, in cui " una delle tangenti giace sul piano $O s$ ed è distinta dalle rette $a$ ed $s$ e " l'altra è situata fuori di questo piano ed inoltre il ramo tangente al piano " medesimo oscula in $A$ il cono di $\Sigma$ corrispondente al piano $a^{\prime}, l^{\prime \prime}$ di $\Sigma^{\prime \prime}$, e 
d'una superficie algebrica per mezzo di trasform. biraz. dello spazio. 99

"quindi la falda del cono $C_{0}$ tangente lungo a al piano $O s$. La medesima "curva si appoggia, in un punto, oltre $A$, alla retta $s$, in un punto varia" bile a ciascuna delle rette $b_{0}$ e $c_{0}$ e in due punti a ciascuna delle rette $b$ e $c$."

Il piano $a^{\prime}, l^{\prime \prime}$ di $\Sigma^{\prime \prime}$ ha per equazione

$$
a^{\prime \prime}{ }_{2} x^{\prime \prime}{ }_{2}+a^{\prime \prime}{ }_{3} x^{\prime \prime}{ }_{3}=0 \text {, }
$$

e quindi l'equazione del cono che gli corrisponde in $\Sigma \grave{e}$

$$
a^{\prime \prime}{ }_{2} B R+a^{\prime \prime}{ }_{3} C Q=0 \text {. }
$$

Ora questo cono oscula lungo la retta a la falda del cono $C_{0}^{y}$ tangente lungo la medesima retta al piano $O s$.

I teoremi precedenti contengono le proprietà della trasformazione fra $\mathrm{i}$ due spazi $\Sigma$ e $\Sigma^{\prime \prime}$ che saranno applicate in seguito.

\section{$\S 4$.}

17. Suppongasi di avere nello spazio $\Sigma$ una superficie $F$ dell'ordine $n$, la quale possegga soltanto un punto $i^{-p l o} A$ in cui il cono tangente $\Delta$ sia semplice e dotato di una generatrice $s, j^{-p l a}(j \leq i)$, di natura qualsiasi. Un piano condotto ad arbitrio per $s$ taglia quindi la superficie $F$ secondo una curva $f$, la quale possiede in $\mathrm{A}$ un punto $i$-plo tale che $j$ degli $i$ rami passanti per $A$ toccano ivi la medesima retta $s$, ma possono avere con essa e fra loro contatti di differenti ordini; mentre ogni altro piano che passi per $A$ e non per $s$ sega la superficie $F$ secondo una curva, la quale ha in $A$ un punto $i$-plo ordinario.

Fra lo spazio $\Sigma$ in cui è data la superficie $F$ e un secondo spazio $\Sigma^{\prime}$ si stabilisca la trasformazione birazionale studiata nel paragrafo 2 , assumendo il punto singolare $A$ di $F$ e la retta singolare $s$ di $\Delta$ come punta fondamentale $A$ e retta fondamentale $s$ dello spazio $\Sigma$, scegliendo l'altro punto fondamentale $O$ di questo stesso spazio fuori della superficie $F$ e gli altri elementi fondamentali di $\Sigma$ come tutti quelli di $\Sigma^{\prime}$ in modo affatto arbitrario. Nella trasformazione così individuata, alla superficie $F$ di $\Sigma$ corrisponde in $\Sigma^{\prime}$ una superficie $F^{\prime}$, di cui si vogliono ora conoscere le proprietà.

18. In virtù dell'ipotesi che la superficie $F$ possiede in $A\left(x_{2}=x_{3}=x_{4}=0\right)$ un punto $i^{-p 2 o}$, la sua equazione è della forma

$$
F=A_{i} x_{1}^{n-i}+A_{i+4} x_{1}^{n-i-1}+A_{i+2} x_{1}^{n-i-2}+\cdots+A_{n}=0,
$$


essendo $A_{i}, A_{i+1}, A_{i+2}, \ldots, A_{n}$ funzioni razionali intere omogenee delle coordinate $x_{2}, x_{3}, x_{4}$ dei gradi indicati dai loro indici, e quindi in particolare

$$
A_{n}=B_{0} x_{4}^{n}+B_{1} x_{4}^{n-1}+B_{2} x_{4}^{n-2}+\cdots B_{n}
$$

dove $B_{0}, B_{1}, B_{2}, \ldots, B_{n}$ rappresentano funzioni analoghe alle precedenti, ma delle sole coordinate $x_{2}$ e $x_{3}$ e dei gradi $0,1,2, \ldots ; n$ rispettivamente. Inoltre poichè il punto $O\left(x_{1}=x_{2}=x_{3}=0\right)$ è stato scelto fuori della superficie $F$, l'equazione di questa non deve essere soddisfatta quando in essa si ponga $x_{1}=0, x_{2}=0, x_{3}=0$; e perchè ciò abbia luogo è necessario che Ja costante $B_{0}$ sia diversa da zero. Ora nell' equazione precedente si ponga in luogo di $x_{1}, x_{2}, x_{3}, x_{4}$ i loro valori dati dalle formule $(5)$; come equazione della superficie $F^{\prime}$ si ottiene:

$$
\begin{gathered}
F^{\prime \prime}=A_{i}^{\prime} p^{\prime n-i} x_{2}^{\prime}{ }_{2-i} x_{3}^{\prime}{ }^{n-i}+A^{\prime}{ }_{i+1} p^{\prime n-i-1} x_{1}^{\prime} x_{2}^{\prime}{ }^{n-i-1} x_{3}^{\prime}{ }^{n-i-1}+ \\
+A_{i+2}^{\prime} p^{\prime n-i-2} x_{1}^{\prime 2} x_{2}^{\prime}{ }^{n-i-2} x_{3}^{\prime}{ }^{n-i-2}+\cdots+\left(B_{0} r^{\prime n} x_{4}^{\prime}{ }^{n}+B_{1}^{\prime} p^{\prime} r^{\prime n-1} x_{4}^{\prime}{ }^{n-1}+\right. \\
\left.+B_{2}^{\prime} p^{\prime 2} r^{\prime n-2} x_{4}^{\prime}{ }^{n-2}+\cdots+B^{\prime}{ }_{n} p^{\prime}{ }^{n}\right) x_{1}^{\prime}{ }^{n-i}=0,
\end{gathered}
$$

nella quale $A_{i}^{\prime}, A^{\prime}{ }_{i+1}, A^{\prime}{ }_{i+2}, \ldots$, indicano ciò che diventano le funzioni $A_{i}$, $A_{i+1}, A_{i+2}, \ldots$, quando in queste si pongano $p^{\prime} x_{3}^{\prime}, p^{\prime} x_{2}^{\prime}, r^{\prime} x_{4}^{\prime}$ in luogo di $x_{2}, x_{3}, x_{4}$ e $B_{1}^{\prime}, B_{2}^{\prime}, \ldots, B_{n}^{\prime}$ denotano i risultati delle sostituzioni di $x_{3}^{\prime}$ e $x_{2}^{\prime}$ ad $x_{2}$ e $x_{3}$. Questa equazione è del grado $3 n-i$ nelle coordinate $x_{1}^{\prime}, x_{2}^{\prime}$, $x_{3}^{\prime}, x_{1}^{\prime}$ e in essa il termine $B_{0} r^{\prime} x^{\prime}{ }_{1}{ }^{n-i} x^{\prime}{ }_{4}{ }^{n}$ non può mai mancare, perchè come si è osservato sopra, il coefficiente $B_{0}$ è necessariamente diverso da zero. Dunque :

I. "La superficie $F^{\prime}$ è dell'ordine $3 n-i$ e possiede in $O^{\prime}$ un punto " $(2 n-i)^{-p l o}$, in cui il cono tangente si scinde nei due piani fissi $r^{\prime}\left(r^{\prime}=0\right)$ " e $b^{\prime} c^{\prime}\left(x_{1}^{\prime}=0\right)$ contati $n$ ed $n-i$ volte rispettivamente."

L'ordine della superficie $F^{\prime}$ può anche ottenersi ricordando che ad una retta $R^{\prime}$ di $\Sigma^{\prime}$ corrisponde in $\Sigma(7$, III) una cubica gobba $S$, che passa per il punto $A$. Così può calcolarsi il grado di multiplicità del punto $O^{\prime}$ per $F^{\prime}$ tenendo presente $(7, I V)$ che ad una retta di $\Sigma^{\prime}$ condotta per $O^{\prime}$ corrisponde in $\Sigma$ una retta passante per $O$.

Ad una retta $R^{\prime}$ di $\Sigma^{\prime}$ appoggiata in un punto $P^{\prime}$ alla retta fondamentale $a^{\prime}$ corrisponde in $\Sigma(11, \mathrm{II})$ una conica che passa per $A$. Questa incontra la superficie $F$ d'ordine $n$ e dotata in $A$ d'un punto $i-p l o$, in $2 n-i$ punti variabili. Quindi in altrettanti punti variabili la retta $R^{\prime}$ deve incontrare la 
d'una superficie algebrica per mezzo di trasform. biraz. dello spazio. 101

superficie $F^{\prime}$; epperò nel punto $P$ cadono

$$
(3 n-i)-(2 n-i)=n
$$

punti d'intersezione. Dunque:

II. "La superficie $F^{\prime}$ possiede la retta fondamentale $a^{\prime}$ come retta "n-pla"

Ad un purto $P^{\prime}$ della retta $a^{\prime}$ di $\Sigma^{\prime}$ corrisponde in $\Sigma(9$ e $4, \mathrm{VI})$ una retta del piano $b c$ appoggiata alla retta $s$ nel punto $M$, diverso da $A$, in cui questa incontra il piano stesso $b c$. Quella retta taglia la superficie $F$ in $n$ punti $H_{1}, H_{2}, H_{3}, \ldots, H_{n}$ in generale distinti fra loro, $\mathrm{i}$ quali determinano altrettanti piani $\alpha_{1}, \alpha_{2}, \alpha_{3}, \ldots, \alpha_{n}$ passanti per la retta $a$, cui corrispondono in $\Sigma^{\prime}(10$ e $5, I V) n$ piani $\alpha_{1}^{\prime}, \alpha_{2}^{\prime}, \alpha_{3}^{\prime}, \ldots, \alpha_{n}^{\prime}$ passanti per la retta $a^{\prime}$. La conica di $\Sigma$ corrispondente ad una retta $R$ di $\Sigma^{\prime}$ situata sopra una qualunque $\alpha^{\prime}{ }_{k}(k=1,2,3, \ldots, n)$ di questi piani e condotta per il punto $P^{\prime}$ di $a^{\prime}$, passa per il punto $H_{k}$. Quindi essa incontra la superficie $F$ in $2 n-i-1$ punti variabili; epperò la retta considerata $R^{\prime}$ tocca nel punto $P^{\prime}$ la superficie $F^{\prime}$. Dunque i piani tangenti a questa superficie in $P^{\prime}$ sono i piani $\alpha_{1}^{\prime}, \alpha_{2}^{\prime}, \alpha_{3}^{\prime}, \ldots, \alpha_{n}^{\prime}$; e siccome questi sono distinti fra loro al pari dei piani $\alpha_{1}, \alpha_{2}, \alpha_{3}, \ldots, \alpha_{n}$ cui corrispondono, così si ha:

III. "Gli $n$ piani tangenti alla superficie $F^{\prime}$ in un punto generico $P$ "della retta $n$-pla $a^{\prime}$ sono distinti fra loro e variabili col punto $P^{\prime}$."

La superficie $F$ non possiede per ipotesi alcuna singolarità fuori del punto $A$; quindi essa viene tagliata dal piano $b c$ secondo una curva generale dell'ordine $n$, alla quale dunque si possono condurre $n(n-1)$ tangenti dal punto $M$. A queste corrispondono altrettanti punti sopra la retta $a^{\prime}$, in ciascuno dei quali, in virtù di quanto precede, due degli $n$ piani tangenti alla superficie $F^{\prime}$ coincidono; epperò:

IV. "Sulla retta $a^{\prime}$ esistono $n(n-1)$ punti cuspidali."

Ad un piano $\alpha^{\prime}$ di $\Sigma^{\prime}$ condotto ad arbitrio per $a^{\prime}$ corrisponde in $\Sigma^{\prime}$ un piano passante per $a$. Fra $i$ punti di questi due piani ha luogo $(12$, IV) una trasformazione quadratica e la curva $f^{\prime}$ secondo cui il piano $\alpha^{\prime}$ taglia la super. ficie $F^{\prime}$ è la curva corrispondente in questa trasformazione alla curva d'intersezione $f$ del piano a con la superficie $F$. Ora questa curva $f$ è dell' ordine $n$ e possiede in $A$, che è un punto fondamentale del piano $\propto$ nell'anzidetta trasformazione, un punto $i$-plo ordinario, perchè (12) il piano a passante per esso non contiene la retta $s$. Quindi la curva corrispondente $f^{\prime}$ è dell'ordine $2 n-i$ e possiede in $O^{\prime}$ un punto multiplo secondo $n-i$, in cui le tangenti 
coincidono con la retta d'intersezione del piano $\alpha^{\prime}$ con il piano $b^{\prime} c^{\prime}$ ed inoltre ciascuno dei suoi rami passanti per $O^{\prime}$ viene incontrato da questa retta, oltre $O^{\prime}$, in altri $n-i$ punti infinitamente vicini ad $O^{\prime} ;$ infine essa possiede un punto $n^{-p l o}$ ordinario nel punto in cui il suo piano incontra la retta, $s^{\prime}$. Dunque:

V. "Un piano $\alpha^{\prime}$, condotto ad arbitrio per la retta $a^{\prime}$ taglia ulte"riormente la superficie $F^{\prime}$ in una curva dell' ordine $2 n-i$, la quale pos" siede un punto $n^{-p l o}$ ordinario nel punto in cui il suo piano incontra la retta $s^{\prime}$ " e un punto $(n-i) p^{p l o}$ in $O^{\prime}$. Le tangenti alla curva in questo punto coin" cidono tutte con la retta $\mathrm{d}^{\prime}$ intersezione del piano $a^{\prime}$ col piano $b^{\prime} c^{\prime}$ e i suoi "rami passanti per esso hanno in comune con questa retta, oltre $O$ ', gli "stessi $n-i$ punti infinitamente vicini ad $0^{\prime}$."

Fra i piani passanti per la retta $a^{\prime}$ vi sono i piani $a^{\prime} b^{\prime}, a^{\prime} c^{\prime}, a^{\prime} d^{\prime}, a^{\prime} e^{\prime}$; si esamineranno in seguito $(19, \mathrm{~V} ; 24, \mathrm{III} ; 21, \mathrm{~V})$. le sezioni da essi operate nella superficie $F^{\prime}$.

Nella trasformazione quadratica fra $\mathrm{i}$ due piani $\alpha$ ed $\alpha^{\prime}$, alla retta $a^{\prime}$ di $\alpha^{\prime}$ corrisponde in a la retta secondo cui questo piano incontra il piano $b c$. Siccome quest' ultima retta incontra la curva $f$ in $n$ punti in generale distinti; così anche la retta $a^{\prime}$ deve incontrare la curva $f^{\prime}$ in $n$ punti distinti e variabili col piano $\alpha$. Ora dal punto $O$ si possono condurre $n(n-1)$ tangenti alla curva d'intersezione della superficie $F$ col piano $b c$, ciascuna delle quali determina un piano passante per $a$, il quale taglia la superficie $F$ in una curva $f$ che tocca l'anzidetta tangente. Quindi, in virtù dell'osservazione testè fatta, si vede che per la retta $a^{\prime}$ passano $n(n-1)$ piani $\alpha^{\prime}$ ciascuno dei quali taglia $F^{\prime}$ secondo una curva $f^{\prime}$, che incontra la retta $a^{\prime}$ in $n$ punti di cui due coincidono. Chiamando col sig. ZeUThen $\left(^{*}\right)$ questi piani piani stazionari dellar retta $a_{1}$, si ha dunque:

VI. "Per la retta $a^{\prime}$ passano $n(n-1)$ piani stazionari."

19. Ad una retta $R^{\prime}$ di $\Sigma^{\prime}$ appoggiata in un punto $P^{\prime}$ alla retta fondamentale $b^{\prime}$ corrisponde in $\Sigma(11$, III) una conica la quale non passa per il punto $A$, epperò incontra la superficie $F$ in $2 n$ punti variabili. Quindi ripetendo il ragionamento fatto per dimostrare il teorema II del $\mathrm{n}^{\circ}$ precedente, si ha:

I. "La superficie $F^{\prime}$ possiede le rette fondamentali $b$ ' e $c^{\prime}$ come rette * $(n-i)-p l e "$

(*) Recherche des singularites qui ont rapport it wne lroite multiple d'une surface. Hathematische Annalen, Band IV, pag. 1. 
d'una superficie algebrica per mezzo di trasform. biraz. dello spazio. 103

Ad un punto $P^{\prime}$ della retta $b^{\prime}$ corrisponde in $\Sigma(9$ e $4, \mathrm{VI})$ una retta passante per il punto $A$ e situata sul piano $c a$. Questa retta incontra la superficie $F$ in $n-i$ punti, fuori di $A$, i quali determinano $n-i$ piani passanti per la retta $b$, cui corrispondono in $\Sigma^{\prime}$ altrettanti piani passanti per $b^{\prime}$. $\mathrm{Si}$ dimostra come nel caso della retta $a^{\prime}$, che questi $n-i$ piani toccano la superficie $F^{\prime}$ nel punto considerato $P^{\prime}$ sopra $b^{\prime}$. Dunque:

II. "Gli $n-i$ piani tangenti alla superficie $F$ ' in un punto gene" rico $P^{\prime}$ di ciascuna delle rette fondamentali $b^{\prime}$ e $c^{\prime}$ sono tutti distinti fra "loro e variabili col punto $P^{\prime}$."

Il piano $c a$, il quale passa per il punto $A$, ma non per la retta $s$, taglia la superficie $F$ secondo una curva che possiede in $A$ un punto $i$-plo ordinario. Perciò dal punto $A$ si possono condurre alla eurva medesima $n(n-1)-i(i+1)$ tangenti. Quindi:

III. "Sopra ciascuna delle due rette $b^{\prime}$ e $c^{\prime}$ esistono $n(n-1)-i(i+1)$ "punti cuspidali."

Ad un piano $\beta^{\prime}$ di $\Sigma^{\prime}$ condotto per la retta $b^{\prime}$ corrisponde in $\Sigma$ un piano $\beta$ passante per la retta $b$. Fra i punti di questi due piani ha luogo $(12, \mathrm{~V})$ una trasformazione quadratica. Quindi come il teorema $\mathrm{V}$ del $\mathrm{n}^{\circ}$ precedente, si dimostra:

IV. “Un piano $\beta^{\prime}$, o $\gamma^{\prime}$, condotto ad arbitrio per la retta $b^{\prime}$, o $c^{\prime}$, " taglia ulteriormente la superficie $F^{\prime}$ in una curva dell' ordine $2 n$, la quale " possiede un punto $n^{\text {-pla }}$ ordinario nel punto in cui il suo piano incontra la "retta $s^{\prime}$ e un punto $x$-plo in $O^{\prime}$. Le tangenti alla curva in questo punto " coincidono tutte con la retta $d^{\prime}$ intersezione del piano $\beta^{\prime}, 0 \gamma^{\prime}$, col piano $r^{\prime}$, " e i suoi rami passanti per esso hanno in comune con questa retta, oltre $O^{\prime}$; " gli stessi $n$ punti infinitamente vicini ad $O^{\prime}$."

Ad ogni punto $P$ della retta $c$ di $\Sigma$ corrisponde in $\Sigma^{\prime}(9$ e 4 , VI) una retta del piano $a^{\prime} b^{\prime}$ appoggiato alla retta $s^{\prime}$. La retta $c$ incontra la superficie $F$ in $n$ punti distinti; quindi il piano $a^{\prime} b^{\prime}$, taglia la superficie $F^{\prime}$, oltre le rette $a^{\prime}$ e $b^{\prime}$ contate $n$ ed $n-i$ volte rispettivamente $(18$, II e $19, I)$ in altre $n$ rette appoggiate ad $s^{\prime}$. Dunque:

V. "Ciascuno dei piani $a^{\prime} b^{\prime}$ e $a^{\prime} c^{\prime}$ taglia la superficie $F^{\prime}$, oltre le "due rette fondamentali in esse contenute, in altre $n$ rette distinte appog"giate alla retta $s . "$

Fra i piani passanti per la retta $b^{\prime}, 0 c^{\prime}$, vi sono i piani $b^{\prime} d^{\prime}$ e $b^{\prime} c^{\prime}$, oppure $c^{\prime} d^{\prime}$ e $c^{\prime} b^{\prime} \equiv b^{\prime} c^{\prime}$ : si esamineranno in seguito $(20 ; \mathrm{V}$ e $21, \mathrm{VI})$ le sezioni da essi operate nella superficie $F^{\prime}$. 
Nella trasformazione quadratica fra i due piani $\beta$ e $\beta^{\prime}$ alla retta $b^{\prime}$ di $\beta^{\prime}$ corrisponde in $\beta$ la retta secondo cui questo piano incontra il piano $c$ a. Siccome quest'ultima retta incontra la curva $f$ in $n$ punti in generale distinti, così anche la retta $b^{\prime}$ incontra la curva $f^{\prime}$ in $n$ punti distinti e rariabili col piano $\beta^{\prime}$. Ora il piano $c$ a taglia la superficie $F$ secondo una curva dell'or. dine $n$, la quale possiede in $A$ un punto $i$-plo ordinario e alla quale quindi si possono condurre, dal punto $O, n(n-1)-i(i-1)$ tangenti. Dunque:

VI. " Per ciascuna delle rette $b^{\prime} c^{\prime}$ passano $n(n-1)-i(i-1)$ "piani stazionari."

20. Ad una retta $R^{\prime}$ di $\Sigma^{\prime}$ appoggiata in un punto $P^{\prime}$ alla retta $d^{\prime}$ corrisponde in $\Sigma(11, \mathrm{IV})$ una conica, la quale passa per il punto $A$, epperò incontra la superficie $F$ in $2 n-i$ punti variabili. Quindi :

I. "La superficie $F$ " possiede la retta fondamentale $d^{\prime}$ come "retta $n$-pla."

Ad ogni punto $P^{\prime}$ della retta $d^{\prime}$ di $\Sigma^{\prime}$ corrisponde in $\Sigma(9$, III) la retta $d$. Questa incontra la superficie $F$ in $n$ punti distinti per ciascuno dei quali passa una, ed una sola, delle coniche di $\Sigma$ corrispondenti $(12, \mathrm{VII})$ alle rette di $\Sigma^{\prime}$ passanti per il punto $P^{\prime}$ dato sopra $d^{\prime}$ e situate in uno stesso piano $U^{\prime}$. Ciascuna delle coniche così determinate incontra la superficie $F$ in $2 n-i-1$ punti variabili; quindi la retta corrispondente ha in comune con la superficie $F^{\prime} n+1$ punti riuniti in $P^{\prime}$. Dunque dal punto $P^{\prime}$ escono $n$ rette distinte situate sul piano dato $U^{\prime}$, le quali toccano in $P^{\prime}$ la superficie $F^{\prime}$. Queste $n$ rette determinano altrettanti piani passanti per $d^{\prime}$, i quali sono tangenti in $P^{\prime}$ ad $F^{\prime}$; epperò :

II. "Gli $n$ piani tangenti alla superficie $F^{\prime}$ in un punto generico $P^{\prime}$ "della retta $d^{\prime}$ sono tutti distinti fra loro e variabili col punto $P^{\prime}$."

Perchè due degli $n$ piani tangenti alla superficie $F^{\prime}$ in un punto della retta $d^{\prime}$ coincidano, è necessario che coincidano due delle $n$ coniche innanzi considerate, il che non può avvenire perchè gli $n$ punti d'intersezione della retta $d$ con la superficie $F$ sono tutti distinti fra loro. Dunque :

III. "Sulla retta $d^{\prime}$ non esistono punti cuspidali."

Si vedrà in seguito $\left(23\right.$, II) quali sono i piani tangenti alla superficie $F^{\prime}$ nel punto $D^{\prime}$, in cui la retta $d^{\prime}$ si appoggia alla $s^{\prime}$.

Ad un piano $\delta^{\prime}$ di $\Psi^{\prime}$ condotto per la retta $d^{\prime}$ corrisponde in $\Sigma(7$, II e 9, III) un cono quadrico circoscritto al quadrispigolo $0 . a b c d$. Quindi la curva $f^{\prime}$ secondo cui quel piano incontra, oltre $d$, la superficie $F^{\prime}$ corrisponde alla curva d'intersezione $f$ di questo cono con la superficie $F$. Ora questa 
d' una superficie algebrica per mezzo di trasform. biraz. dello spazio. 105

curva $f$ si appoggia alla retta $d$ negli $n$ punti in cui questa incontra la superficie $F$, i quali sono tutti distinti fra loro. Percio sono tali anche gli $n$ punti nei quali la curva medesima si appoggia alla generatrice $x$ del cono anzidetto infinitamente vicina a $d$. La curva $f^{\prime}$ incontra la retta $x^{\prime}$ del piano $\gamma^{\prime}$, corrispondente a $x$, in $n$ punti che sono i corrispondenti di quelli comuni alla retta * ed alla curva $f$, epperò, come questi, sono tutti distinti fra loro. Quindi sono tali anche gli $n$ punti nei quali la curva $f^{\prime}$ incontra la retta $d^{\prime}$. Dunque un piano condotto ad arbitrio per la retta $d^{\prime}$ taglia la superficie $F^{\prime}$ secondo una curva $f^{\prime}$, la quale incontra la retta $d^{\prime}$, fuori di $O^{\prime}$ e $D^{\prime}$, in $n$ punti distinti fra loro. Si vedrà in seguito $(25$, II) quale sia la singolarità che questa curva possiede nel punto $O^{\prime}$. Intanto si noti ancora che non può mai accadere che due degli $n$ punti di incontro di $f^{\prime}$ con $d^{\prime}$, fuori di $O^{\prime}$, coincidano fra loro; e quindi si ha:

IV. "Per la retta $d^{\prime}$ non passano piani stazionari."

Il piano $d^{\prime} b^{\prime}$ di $\Sigma^{\prime}$ corrisponde al piano $d b$ di $\Sigma$, il quale taglia la superficie $F$ secondo una curva generale $f$ dell' ordine $n$. Siccome fra i punti dei due piani $d b$ e $d^{\prime} b^{\prime}$ ha luogo (12, VI) una corrispondenza omografica, cosi alla curva $f$ del piano corrisponde sopra il secondo una curva $f^{\prime}$, affatto generale dell' ordine $n$, e questa insieme alle rette $b^{\prime}$ e $d^{\prime}$ contate $(19, \mathrm{I}$ e 20 I) $n-i$ ed $n$ volte rispettivamente costituisce la completa intersezione del piano $b^{\prime} d^{\prime}$ con la superficie $F^{\prime}$ Dunque:

V. "Ciascuno dei due piani $d^{\prime} b^{\prime}$ e $d^{\prime} c^{\prime}$ taglia la superficie $F^{\prime \prime}$, "oltre le due rette fondamentali in esso contenate, secondo una curva gene" rale dell' ordine $n$."

Si esamineranno in seguito (24, III 22, VI) le sezioni fatte nella superficie $F^{\prime}$ dai piani $d^{\prime} a^{\prime}$ e $d^{\prime} s^{\prime}$.

21. Ad una retta di $\searrow^{\prime}$ appoggiata alla retta fondamentale $e^{\prime}$ corrisponde in $\Sigma(11, \mathrm{~V})$ una conica la quale non passa per $A$ e quindi incontra la superficie $F$ in $2 n$ punti variabili. Dunque:

I. " La superficie $F^{\prime}$ possiede la retta fondamentale $e^{\prime}$ come retta " $(n-i)^{-p l a} . "$

Ad ogni punto $P^{\prime}$ della retta $e^{\prime}$ corrisponde in $\Sigma(9$, III) la retta $a$. Questa incontra la superficie $F$ in $n-i$ punti, fuori di $A$, tutti distinti frat loro, per ciascuno dei quali passa una, ed una sola, delle coniche di $\Sigma$ corrispondenti $\left(12\right.$, VIII) alle rette di $\Sigma^{\prime}$ passanti per il punto $P^{\prime}$ dato sopra $e$ e situate in un medesimo piano. Quindi come $\mathrm{i}$ teoremi II e III del n..$^{\circ}$ che precede si hanno i seguenti:

Annali di Matematica, tomo XXV. 
II. "Gli $n-i$ piani tangenti alla superficie $F^{\prime}$ in un punto ge" nerico $P^{\prime}$ della retta $e^{\prime}$ sono tutti distinti fra loro e variabili col punto $P^{\prime}$." III. "Sulla retta $e^{\prime}$ non esistono punti cuspidali."

Si vedrà in seguito $(23, \mathrm{~V})$ quali sono i piani tangenti alla superficie $F^{\prime}$ nel punto $E^{\prime}$, in cui la retta $e^{\prime}$ si appoggia alla $s^{\prime}$.

Ad un piano $\varepsilon^{\prime}$ condotto per la retta $e^{t}$ corrisponde in $\Sigma(7,11$ e 9, III $)$ un cono quadrico circoscritto al triedro $O . a b c$ e tangente lungo la generatrice $a$ al piano $t$. Quindi la curva $f^{\prime}$ secondo cui quel piano incontra, oltre $e^{\prime}$, la superficie $F^{\prime}$, corrisponde alla curva d'intersezione $f$ di questo cono con la superficie $F$. Ora questa curva $f$ si appoggia alla retta $a$, oltre $A$, negli $n-i$ punti in cui la retta stessa incontra la superficie $F$, i quali sono tutti distinti fra loro. Quindi ripetendo il ragionamento fatto per dimostrare il teorema IV del n. ${ }^{\circ}$ precedente, si trova che la curva $f^{\prime}$ incontra la retta $e^{\prime}$, fuori di $O^{\prime}$ ed $E^{\prime}$ in $n-i$ punti distinti fra loro. Si vedrà in seguito $(25$, III) quali siano le singolarità che questa curva possiede nei punti $O^{\prime}$ ed $E^{\prime}$; intanto però si può concludere:

IV. "Per la retta $e^{\prime}$ non passano piani stazionari."

Il piano $a^{\prime} e^{\prime}$ corrisponde al piano $t$, e fra $\mathrm{i}$ punti di questi due piani ha luogo una corrispondenza omografica $(12, \mathrm{VI})$. Ora il piano $t$ taglia la superficie $F$ secondo una curva dell'ordine $n$, la quale possiede in $A$ un punto $i^{i}$-plo ordinario. La curva $f^{\prime}$ a questa corrispondente nel piano $a^{\prime} e^{\prime}$, nell' anzidetta omografia, è quindi una curva dell'ordine $n$, la quale possiede in $E^{\prime}$ un punto $i^{-p l o}$ ordinario. Questa curva insieme alle rette $a^{\prime}$ ed $e^{\prime}$ contate $(18, I$ e 21, I) $n$ ed $n-i$ volte rispeitivamente, costituisce la completa intersezione del piano $a^{\prime} e^{\prime}$ con la superficie $F^{\prime}$. Dunque:

V. "Il piano $a^{\prime} e^{\prime}$ taglia la superficie $F^{\prime}$, oltre le due rette fonda" mentali in esso contenute, secondo una curva dell' ordine $n$, la quale pos" siede in $E^{\prime}$ un punto $i$-plo ordinario."

Fra i piani passanti per la retta $e^{\prime} \nabla^{\prime}$ è ancora il piano $b^{\prime} c^{\prime}$. Ora si sa $(10,11)$ che fra i punti di questo piano di $\Sigma^{\prime}$ e le rette della stella $(A)$ di $\Sigma$ ha loogo una trasformazione quadratica, per la quale una delle rette fondamentali della stella è la retta $s$, cui corrisponde sul piano la retta $a_{1}$. In virtù di questa trasformazione, al cono $\Delta$, d'ordine $i$ tangente in $A$ alla superficie data $F$ e avente la retta $s$ come generatrice $j$-pla, di natura qualsiasi, corrisponde sul piano $b^{\prime} e^{\prime}$ una curva di $\Delta^{\prime}$ dell' ordine $2 i-j$, la quale possiede nel punto fondamentale $E^{\prime}$ un punto $i^{-p t_{0}}$ ordinario e in $O^{\prime}$ un punto $(i-j)^{-p l o}$, in cui tutte le tangenti coincidono con la retta $a_{1}$, e questa in- 
d' una superficie algebrica per mezzo di trasform. biraz. dello spazio. 107

contra la curva $\Delta^{\prime}$ nel punto $O^{\prime}$, il quale conta per $2(i-j)$ punti d'intersezione, in altri $j$ punti, i quali possono coincidere e formare anche un punto $i^{-p l o}$. Nella stessa trasformazione, al cono $\Delta$ corrisponde sul piano $b^{\prime} c^{\prime}$, non solo la curva $\Delta^{\prime}$, ma anche la retta $a_{i}$, contata $j$ volte, perchè quel cono possiede la retta $s$ come generatrice $j$-pla. Questa retta $a_{1}$, contata $j$ volte, e l'anzidetta curva $\Delta^{\prime}$ fanno parte dell' intersezion 3 del piano $b^{\prime} c^{\prime}$, con la superficie $F^{\prime}$, la quale intersezione viene completata dalle rette $b^{\prime}, c^{\prime}, e^{\prime}$, ciascuna contata $n-i$ volte $(19$, I e $21, I)$. Dunque:

VI. "Il piano $b^{\prime} c^{\prime}$ taglia la superficie $F^{\prime}$, oltre le tre rette fonda" mentali $b^{\prime}, c^{\prime}, e^{\prime}$ in esso contenute, e la retta $a_{1}$ contata $j$ volte, secondo " una curva $\Delta^{\prime}$ dell'ordine $2 i-j$, la quale possiede in $E^{\prime}$ un punto $i$-plo " ordinario e in $O^{\prime}$ un punto $(i-j)^{-p l o}$, in cui le tangenti coincidono tutte " con la retta $a_{1}$. Ciascun ramo di $\Delta^{\prime}$ passante per $O$ incontra ivi la retta $a_{3}$, "oltre $O$, che deve essere contato $i-j$ volte, in altri $i-j$ punti infinita"mente vicini ad $O^{\prime}$; e la curva $\Delta^{\prime}$ incontra la stessa retta $a_{1}$, fuori di $O^{\prime}$, " in altri $j$ punti, i quali possono anche formare un punto $j$-plo."

22. Ad una retta $R^{\prime}$ di $\Sigma^{\prime}$ appoggiata in un punto $P^{\prime}$ alla retta $s^{\prime}$ corrisponde in $\Sigma(11, \mathrm{I})$ una conica, la quale passa per il punto $A$, e quindi incontra la superficie $F$ in $2 n-i$ punti variabili. Dunque:

I. "La superficie $F^{\prime}$ possiede la retta fondamentale $s^{\prime}$ come retta $n$-plo."

Ad un punto qualunque $P^{\prime}$ della retta $s^{\prime}$ corrisponde in $\Sigma(9$ e $4, \mathrm{~V})$ una generatrice del cono $C$, la quale incontra la superficie $F$ in $n$ punti in generale distinti fra loro, i quali determinano $n$ piani passanti per la retta $s$, cui corrispondono in $\Sigma^{\prime}(10 \mathrm{e} 5, \mathrm{I})$ altrettanti piani passanti per $s^{\prime}$, i quali toccano $F^{\prime}$ nel punto $P^{\prime}$. Dunque:

II. "Gli $n$ piani tangenti alla superficie $F^{\prime}$ in un punto generico $P^{\prime}$ "della retta $s^{\prime}$ sono tutti distinti fra loro e variabili col punto $P^{\prime}$."

Fra le generatrici del cono $C$ ve ne sono alcune (il numero delle quali è determinato in ogni caso) che riescono tangenti alla superficie $F$. A ciascuna di queste corrisponde sulla retta $s^{\prime}$ un punto $P^{\prime}$ in cui due degli $n$ piani tangenti alla superficie $F^{\prime}$ coincidono. Dunque:

III. "Sulla retta $s$ esistono tanti punti cuspidali quante sono le "generatrici del cono $C^{\prime}$, distinte dalla retta $O A$, che toccano la super"ficie $F . "$

Ad un piano $\sigma^{\prime}$ di $\Sigma^{\prime}$ condotto ad arbitrio per la retta $s^{\prime}$ corrisponde in $\Sigma$ un piano passante per $s$. Fra i punti di questi due piani ha luogo $(12$, I) una trasformazione quadratica per la quale sono fondamentali sul 
piano $\sigma$ il punto $A$ e i punti $B$ e $C$ in cui questo piano incontra le rette $b$ e $c$, e sul piano $\sigma^{\prime}$ i punti $A^{\prime}, B^{\prime}, C^{\prime}$ in cui questo incontra le rette $a^{\prime}, b^{\prime}, c^{\prime}$. Inoltre in questa trasformazione alla retta $s$ di $\sigma$ corrisponde sul piano $\sigma^{\prime}$ la retta in cui questo taglia il piano $a^{\prime} d^{\prime}\left(r^{\prime}=0\right)$; e alla retta $s^{\prime}$ di $\sigma^{\prime}$, la conica secondo la quale $\sigma^{\prime}$ sega il cono $C$. Ora la curva d'intersezione $f^{\prime}$ del piano $\sigma^{\prime}$ con la superficie $F^{\prime}$ è la curva di $\sigma^{\prime}$ corrispondente nell'anzidetta trasformazione alla curva d'intersezione $f$ del piano $\sigma$ con la superficie $F$. Questa curva $f$ è, per le ipotesi fatte (17) sulla superficie $F$, dell'ordine $n$ e possiede in $A$ un punto $i$-plo tale che $j$ degli $i$ rami passanti per esso toccano ivi la medesima retta $s$, ma possono avere con essa contatto di differenti ordini. Perciò la curva $f^{\prime}$ è dell'ordine $2 n-i$, possiede in $A^{\prime}, B^{\prime}, C^{\prime}$ punti multipli ordinari secondo i numeri $n, n-i, n-i$ rispettivamente (tutto ciò è in accordo col teorema I di questo $\mathrm{n}^{\circ}$, col teorema II del n. ${ }^{\circ} 19$ e con quello I del n.. $\left.{ }^{\circ} 19\right)$ e incontra la retta $B^{\prime} C^{\prime}$ in $i$ punti, dei quali $i-j$ sono quelli in cui questa retta taglia, fuori di $E^{\prime}$, la curva $\Delta^{\prime}(21, \mathrm{VI})$ e i rimanenti $j$ coincidono nel punto $A_{1}$, intersezione della retta $a_{1}$ col piano $\sigma^{\prime}$. Questi $j$ punti coincidenti possono nascere dal fatto che $A_{1}$ sia un punto $j$-plo della curva $f^{\prime}$; e siccome questo è il caso più sfavorevole che possa presentarsi, cos̀ si supporrà d'ora in avanti che esso abbia luogo. Inoltre si osservi che i punti comuni alla curva $f^{\prime}$ e alla retta $s$ sono i corrispondenti di quelli nei quali la curva $f$ incontra la conica d'intersezione del piano a col cono $C$, epperò al pari di questi sono in generale tutti distinti fra loro. Dunque:

IV. "Un piano condotto ad arbitrio per la retta $s^{\prime}$, taglia la su" perficie $F^{\prime}$ oltre la retta stessa $s^{\prime}$ secondo una curva dell' ordine $2 n-i$, "la quale possiede in $A^{\prime}, B^{\prime}, C^{\prime}$ punti multipli secondo i numeri $n, n-i$, u $n-i$ rispettivamente, in $A_{1}$ un punto $j$-plo di natura incognita, ed incontra " la retta $s$ in $2 n-i$ punti in generale distinti fra loro."

Se la curva $f^{\prime}$ tocca la retta $s^{\prime}$, la curva corrispondente $f$ tocea il cono $C$, e viceversa. Dunque:

V. "Per la retta $s^{\prime}$ passano tanti piani stazionari quante sono le " sezioni fatte nella superficie $F$ con piani condotti per la retta $s$, le quali "riescono tangenti al cono $C^{\prime}$."

Il piano $O^{\prime} s^{\prime}$ contiene le tre rette fondamentali $d, e^{\prime}, s^{\prime}$. Siccome queste sono multiple per $F^{\prime}$ secondo i numeri $n, n-i$ ed $n$ rispettivamente, così il piano $O^{\prime} s^{\prime}$ non incontra la superficie $F^{\prime}$, oltre le rette medesime; epperò :

VI. "Il piano $O^{\prime} s^{\prime}$ taglia la superficie $F^{\prime}$ soltanto nelle rette "d, $e^{\prime}, s^{\prime}$ contate $n, n-i, n$ volte rispettivamente." 
d' una superficie algebrica per mezzo di trasform. biraz. dello spazio. 109

23. Ad una retta di $\Sigma^{\prime}$ condotta per il punto $D^{\prime}$ e non situata sul piano $O^{\prime} s^{\prime}$, corrisponde in $\Sigma(12, I I)$ una conica la quale passa per il punto $A$ e quindi incontra la superficie $F$ in $2 n-i$ punti variabili. Dunque:

I. "La superficie $F^{\prime}$ possiede in $D^{\prime}$ un punto $n$-plo."

Fra le coniche di $\Sigma$ corrispondenti alle rette di $\Sigma^{\prime}$ condotte per il punto $D^{\prime}$ e non situate sul piano $O^{\prime} s^{\prime}$, non ve n'ha alcuma che si appoggi alla retta $d$; quindi nessuna di queste rette tocea nel punto $D^{\prime}$ la superficie $F^{\prime}$. Però ogni retta condotta per il punto $D^{\prime}$ e situata sul piano $O^{\prime} s^{\prime}$ è tangente in $D^{\prime}$ ad $F^{\prime}$. Infatti, il piano $O^{\prime} s^{\prime}$ taglia la superficie $F^{\prime}$ secondo le rette $s^{\prime}, d^{\prime}, e^{\prime}$ soltanto $(22, \mathrm{VI})$. Quindi ogni retta che passi per $D^{\prime}$ e giaccia sopra $O^{\prime} s^{\prime}$, incontra la superficie $F^{\prime}$, fuori di $D^{\prime}$, soltanto nel punto in cui essa taglia la retta $e^{\prime}$. Ma perchè questa è $(n-i)$-pla per $F^{\prime}$, quel punto conta per $n-i$ intersezioni della retta considerata con la superficie $F^{\prime}$; le rimanenti $(3 n-i)-(n-i)=2 n$ cadono dunque nel punto $D^{\prime}$. Quindi :

II. "Gli $n$ piani tangenti alla superficie $F^{\prime}$ nel punto $D^{\prime}$ coincidono u col piano $O^{\prime} s^{\prime}$, e ogni retta di questo piano condotta per $D^{\prime}$ ba in comune " con la superficie $2 n$ punti riuniti in $D^{*}$ *

Ad un piano di $\Sigma^{t}$ condotto per il punto $D$ corrisponde in $\Sigma(7,1)$ una superficie del $3 .^{\circ}$ ordine $\varphi$, la quale $(9$ e $4, \nabla)$ contiene la retta $d$ e tocca lungo questa il cono $C$. Questa superficie incontra la superficie data $F$ secondo una curva $f$, la quale si appoggia a $d$, negli $n$ punti in cui questa sega F. Alla curva $f$ corrisponde la curva d'intersezione $f$ del piano considerato con la superficie $F^{\prime}$, e agli $n$ rami di quella appoggiati alla retta $d$, oorrispondono gli $n$ rami di questa passanti per il punto $D^{\prime}$, ciascuno dei quali ha in comune col piano tangente $O^{\prime} s^{\prime}$, oltre gli $n$ punti riuniti in $D^{\prime}$, altri $n$ punti infinitamente vieini a $D^{\prime}$, nou essendovi aleuna ragione perchè un ramo abbia con l'anzidetto piano un contatto più intimo di un altro. Dunque :

III. "Un piano condotto ad arbitrio per il punto $D$ taglin la su"perficie $F^{\prime}$ secondo una curva che possiede in $D$ un punto $n$-plo in cui le " tangenti coincidono con la retta d intersezione del piano dato col piano $O^{\prime} s^{\prime}$ " ed ogni ramo passarte per esso ha in comune con la superficie $F^{*}$, oltre

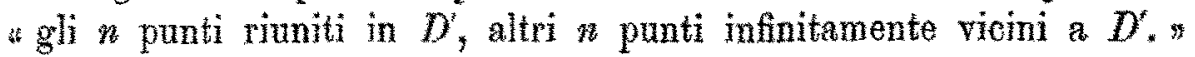

Ad una retta di $\Sigma^{\prime}$ condotta per il punto $E^{\prime}$ e non situata sul piano $O^{\prime} s^{\prime}$ corrisponde in $\mathrm{\Sigma}(12, \mathrm{III})$ una conica, la quale passa per il punto $A$ e quindi incontra la superficie $F$ in $2 n-i$ punti variabili. Dunque:

IV. "La superficie $F^{\prime}$ possiede in $E^{\prime}$ un punto $n^{-w_{*}^{*} *}$ 
Il piano $t$ non passa per la retta $s$; epperò (12) taglia la superficie $F$ secondo una curva la quale possiede in $A$ un punto $i-p l o$ con le tangenti tutte distinte fra loro e tali che ciascuna ha in comuno con la superficie $F$, $i+1$ punti riuniti in $A$. Queste $i$ tangenti determinano altrettanti piani pas. santi per la retta $s$, e fra le coniche di $\Sigma$ corrispondenti alle rette di $\Sigma^{\prime}$ condotte per il punto $E$, le quali coniche sono tangenti in $A$ al piano $t$, soltanto quelle situate in una qualsiasi degli $i$ piani anzidetti, incontrano la superficie $F$ in $2 n-(i+1)$ punti variabili. Quindi fra le rette di $\Sigma^{\prime}$ condotte per il punto $E$, fuori del piano $O^{\prime} s^{\prime}$, soltanto quelle contenute negli $i$ piani di $\Sigma^{\prime}$ corrispondenti agli $i$ piani di $\Sigma$ ora considerati, toccano in $E^{\prime}$ la superficie $F^{\prime}$. Questi $i$ piani di $\Sigma^{\prime}$ passanti per $s^{\prime}$ e tutti distinti fra loro al pari dei piani di $\Sigma$ cui corrispondono, sono dunque tangenti in $E^{\prime}$ alla superficie $F^{\prime}$. Ripetendo poi il ragionamento fatto sopra per dimostrare il precedente teorema II, si trova che anche il piano $O^{\prime} s^{\prime}$ tocca in $E^{\prime}$ la superficie $F^{\prime}$ e che ogni retta di questo piano condotta per il punto $E^{\prime}$, ha in comune con la superficie $F^{\prime}$, oltre gli $n$ punti riuniti in $E^{\prime}$, altri punti infinitamente vicini ad $E^{\prime}$. Quindi raccogliendo si ha:

V. "Degli $n$ piani tangenti alla superficie $F^{\prime}$ nel punto $E^{\prime}$, $i$ sono "distinti fra loro e i rimanenti $n-i$ coincidono col piano $O^{\prime} s^{\prime}$. Inoltre ogni " retta di questo piano condotta per il punto $E^{\prime}$ ha in comune con la super" ficie $F^{\prime}$, oltre gli $n$ punti riuniti in $E^{\prime}$, altri $n-i$ punti infinitamente vi"cini ad $E^{\prime} . "$

Ad un piano di $\Sigma^{\prime}$ condotto ad arbitrio per il punto $E^{\prime}$ corrisponde in $\Sigma$ una superficio del $3 .^{\circ}$ ordine $\varphi$, la quale possiede in $A$ un punto doppio biplanare, invece di un punto doppio conico, perchè il piano di $\Sigma^{\prime}$ cui corrisponde taglia il piano $b^{\prime} c^{\prime}$ secondo una retta passante per $E^{\prime}(10$, II). Questa superficie incontra la superficie data $F$ secondo una curva $f^{\prime}$, la quale si appoggia alla $a$, fuori di $A$, negli $n-i$ punti distinti, in cui questa retta sega $F$. Quindi come il precedente teorema III, si trova:

VL. "Un piano condotto ad arbitrio per il punto $E^{\prime}$ taglia la su" perficie $F^{\prime}$ secondo una curva che possiede in $E_{i}^{\prime}$ un punto $n_{-}$plo, in cui $i$ " delle $n$ tangenti sono fra loro distinte e le rimanenti $n-i$ coincidono con la " retta d'intersezione del piano dato col piano $O^{\prime} s^{\prime}$ ed ogni piano passante per " esso e tangente a quest' ultima retta, ha in comune con la superficie $F^{\prime}$ oltre "gli $n$ punti riuniti in $E^{\prime}$, altri $n-i$ punti infinitamente vieini ad $E^{\prime}$ " 24. Si è fatta l'ipotesi $(22$, IV) che un piano arbitrario $\sigma$ condotto per la retta $s^{\prime}$ tagli la superfieie $F^{\prime \prime}$ secondo una curva $f^{\prime}$, la quale possegga 
d' una superficie algebrica per mezzo di trasform. biraz. dello spazio. 111

in $A_{1}$ un punto $j-p l o$. Al piano $\sigma^{\prime}$ di $\Sigma^{\prime}$ corrisponde in $\Sigma$ un piano $\sigma$ passante per la retta $s$ e in virtù della trasformazione quadratica che ha luogo fra questi due piani, alle rette di $\sigma^{\prime}$ passanti per il punto $A_{1}$ corrispondono le coniche di $\sigma$ circoscritte al triangolo fondamentale $A B C$ e tangenti in $A$ alla retta $s$. Ciascuna retta di quel fascio, la quale non tocchi in $A_{1}$ la curva $f^{\prime}$ incontra la curva stessa, e quindi anche la superficie $F^{\prime}$ in $2 n-i-j$ punti variabili; perciò la conica corrispondente deve incontrare in altrettanti punti variabili la superficie $F$, epperò dei $2 n$ punti che essa ha in comune con questa superficie, $i+j$ debbono cadere in $A$. Ammesso (ciò che sembra evidente) che il numero dei punti d'intersezione riuniti in $A$, della superficie $F$ con una curva passante per $A$, non dipenda dall' ordine di questa, ma soltanto dal suo modo di comportarsi rispetto ad $F$ nelle immediate vicinanze di $A$, si può concludere:

I. "Se una curva passa semplicemente per il punto $A$ e tocca ivi "la retta $s, i+j$ dei punti che essa ha in comune con la superficie $F$ ca"dono in $A . "$

Ad una retta di $\Sigma^{\prime}$ appoggiata alla retta $a_{1}$, corrisponde in $\Sigma(11, \mathrm{VI})$ una cubica gobba, la quale passa per il punto $A$ e tocca ivi la retta $s$. Quindi, in virtù del teorema precedente, essa incontra la superficie $F$ in $3 n-(i+j)$ punti variabili; epperò :

II. "La superficie $f^{\prime}$ possiede la retta $a_{1}$ come retta $j$-pia."

I piani tangenti alla superficie $F^{\prime}$ in un punto generico di questa retta $a_{4}$ sono incogniti; però si possono conoscere alcune delle proprietà delle sezioni operate in $F^{\prime}$ dai piani passanti per $a_{1}$.

Fra questi piani v'è intanto il piano $a^{\prime} d^{\prime}\left(r^{\prime}=0\right)$, il quale taglia la superficie $l^{\prime \prime}$ nelle rette $a^{\prime}$ e $d^{\prime}$, ciasouna delle quali deve essere contata $n$ volte (18, II e 20, I), e in tante rette passanti per il punto $O^{\prime}$, quanti sono i punti in cui la retta $s$ incontra, fuori di $A$, la superficie $F(9$, IV). Ora nell'ipotesi fatta che ogni sezione $f$ operata in $F$ da un piano $\sigma$, passante per $s$, si trasformi in una curva $f^{\prime}$ del piano $\sigma^{\prime}$, corrispondente a $\sigma$, la quale possegga in $A_{4}$ un punto $j^{-p l o}$, segue che la retta $s$ ha in comune con la superficie $F$, fuori di $A, n-i-j$ punti. Infatti, la curva $f^{\prime}$ dell'ordine $2 n-i$ possiede nel punto $A^{\prime}$, intersezione del piano $\sigma^{\prime}$ con la retta $a^{\prime}$, un punto $u^{-p l o}(18$, II $)$ $e$ in $A_{1}$ un punto $j-p l o$ per ipotesi. Quindi la retta in oui il piano $\sigma^{\prime}$ viene segato dal piano $a^{\prime} d^{\prime}$, incontra la curva $t^{\prime}$, fuori di $A^{\prime}$ e di $A_{\curlywedge}$, in $(2 n-i)-(n+j)=n-i-j$ punti, poichè in virtù dell'arbitrarietà che vi è nella scelta degli elementi fondamentali di $\Sigma^{\prime}$, può sempre farsi in modo 
che l'anzidetta retta non riesca tangente in $A_{1}$ alla curva $f^{\prime}$. Ma alla retta medesima corrisponde sul piano $\sigma$ la retta $s$; dunque quest'ultima retta incontra la curva $f$, epperò anche la superficie $F$ in $n-i-j$ punti. Quindi :

III. "Il piano $a^{\prime} d^{\prime}$ taglia la superficie $F^{\prime}$, oltre le rette $a^{\prime}, d^{\prime}, a_{1}$, in altre $n-i-j$ concorrenti nel punto $O^{\prime} . "$

Ad un piano $V^{\prime}$ di $\Sigma^{\prime}$ condotto per la retta $a_{4}$ corrisponde in $\Sigma$ un cono quadrico $V$ circoscritto al triedro $0 . a b c$ e tangente lungo la retta $a$ al piano $O s$. Questo cono taglia la superficie data $F$ secondo una curva $f$ dell'ordine $2 n$, la quale possiede in $A$ un punto $j^{-p l o}$, in cui le tangenti sono le rette secondo le quali il piano $O s$, sega il cono tangente in $A$ alla superficie $F$, epperò secondo le ipotesi fatte intorno a questo cono, $i-j$ sono distinte fra loro e dalla retta $s$, mentre le rimanenti $j$ coincidono con questa retta. Inoltre la medesima curva $f$ si appoggia alla retta $a$ negli $n-i$ punti in cui questa incontra, fuori di $A$, la superficie $F$, e in ciascuno di essi tocca il piano $O s$. La curva $f$ corrisponde in $\Sigma$ alla curva $f^{\prime}$ di $2^{\prime}$, secondo la quale il piano dato $V^{\prime}$ taglia, fuori di $a_{1}$, la superficie $F^{\prime}$.

Ciascuno dei $j$ rami della curva $f$ passanti per il punto $A$ e ivi tangenti alla retta $s$, ha un piano osculatore $\sigma$ passante per la stessa retta $s$. A questo piano corrisponde in $\Sigma^{\prime}$ un piano $\sigma^{\prime}$ passante per la retta $s^{\prime}$, il quale incontra la curva $f^{\prime}$ in un numero di punti variabili di un'unità almeno inferiore a quello secondo cui l'incontra un piano condotto ad arbitrio per $s$, perchè altrettanto accade del numero dei punti d'intersezione del piano $\sigma$ con la curva $f$. Il piano $\sigma^{\prime}$ sega la retta $a_{1}$ in un punto $A$, ed è in questo che va a cadere quell' ulteriore punto fisso d'intersezione di $\sigma^{\prime}$ con $f^{\prime}$, poichè nella trasformazione quadratica che ha luogo fra i punti dei due piani o e $\sigma^{\prime}$, al punto della retta $s$ infinitamente vicino ad $A$, corrisponde il punto $A_{1}$. La curva $f^{\prime}$ passa danque per questo punto; eppero si ha:

TV. "Un piano condotto ad arbitrio per la retta $a_{1}$ taglia ulterior“ mente la superficie $F^{\prime}$ secondo una curva $f^{\prime}$, la quale incontra $a_{4}$, fuori * di $O^{\prime}$, in $j$ punti, i quali sono distinti oppure tutti od in parte coincidenti, " secondochè tali sono $\mathrm{i}$ piani osculatori in $A$ alla curva $f$."

Enunciando cosi come si è fatto questo teorema, si è ammesso che i piani osculatori in $A$ alla curva $f$ siano distinti dal piano $0 s$. Se alcuni di essi, o anche tutti, coincidono con questo piano, alcuni dei punti $j$, o anche tutti, vanno a coincidere con $O^{\prime}$. Questo caso, per ora, viene escluso. 
d' una superficie algebrica per mezzo di trasform. biraz. dello spazio. 113

25. Un piano $V^{\prime}$ condotto ad arbitrio per il punto $O^{\prime}$ taglia la superficie $F^{\prime}$ secondo una curva $f^{\prime}$, la quale possiede $(22, I)$ un punto $n$-plo ordinario nel punto $S^{\prime}$ in cui jl piano $V^{\prime}$ incontra la retta $s^{\prime}$, e $(18, I)$ un punto $(2 n-i)^{-p l o}$ in $O^{\prime}$, in cui $n$ delle tangenti coincidono nella retta d'intersezione di $V^{\prime}$ col piano $r^{\prime}$ e le rimanenti $n-i$ nella retta d' intersezione dello stesso $V^{\prime}$ col piano $b^{\prime} c^{\prime}$.

$\Lambda$ piano $V^{\prime}$ corrisponde in $\Sigma(7$, II) un cono quadrico $\Gamma$ circoseritto al triedro $O . a b c$, il quale taglia la superficie data $F$ secondo una curva $f^{\prime}$, che è precisamente la curva di $\Sigma$ corrispondente all' anzidetta sezione piana $f^{\prime}$, di $F^{\prime}$. La curva $f$ è dell' ordine $2 n$ e possiede in $A$ un punto $i$-plo ordinario, perebè il punto $A$ è un punto semplice per il cono $\Gamma$, e un punto $i$-plo per la superficie $F$, e inoltre il piano tangente in $A$ a $\Gamma$ non contenendo la retta $s$, sega il cono $\Delta$ tangente nello stesso punto $A$ ad $F$, in $i$ rette tutte distinte fra loro. La medesima curva $f$ non passa per $O$, e non si appoggia nè alla retta $d$, nè alla retta $s$, mentre si appoggia in $n-i$ punti distinti, fuori di $A$ e di $O$, alla retta $a$, in ciascuno dei quali tocca il piano tangente lungo $a$ al cono $\Gamma$, e in $n$ punti, fuori di $O$, pure distinti fra loro, a ciascuna delle rette $b, c$, ed $m$, dicendo $m$ la retta secondo la quale il cono $\Gamma$ viene tagliato, oltre $a$, dal piano $O s$. Agli $n$ rami della curva $f$ appoggiati alla retta $m$, corrispondono $(9, \mathrm{I})$ gli $n$ rami della curva $f^{\prime}$ tangente in $O^{\prime}$ al piano $r^{\prime}$; e agli $n-i$ rami di quella appoggiati alla retta $a$, corrispondono gli $n-i$ rami di questa tangenti in $O^{\prime}$ al piano $b^{\prime} c^{\prime}$. Il piano $r^{\prime}$ incontra $(24$, III) la superficie $F^{\prime}$ soltanto in rette concorrenti nel punto $O^{\prime}$, epperò ogni retta del piano stesso condotta per $O^{\prime}$ non incontra la superficie $F^{\prime}$ fuori di $O^{\prime}$. Della stessa proprieta gode dunque la tangente in $O^{\prime}$ alla curva $f^{\prime}$ e situata sul piano $r^{\prime}$; epperò questa tangente incontra la curva $f^{\prime}$ oltre il punto $O^{\prime}$, che è $(2 n-1)-p h o$ per $f^{\prime}$ in altri $(3 n-i)-(2 n-i)=n$ punti infinitamente vicini ad $O^{\prime}$. E siccome non $\nabla^{\prime}$ è alcuna ragione per cui uno degli $n$ rami di $f^{\prime}$ tangenti in $O^{\prime}$ al piano $r^{\prime}$ si comporti in $O^{\prime}$ in un modo diverso di un altro ramo, cost si può coneludere che tutti questi $n$ rami si toccano in $O^{\prime}$, o meglio in un punto a questo infinitamente vicino del piano $r$, ed harino jvi un contatto $n$ - punto. In modo affatto analogo, tenendo presente il teorema VI del n. ${ }^{\circ} 21$, si conclude che gli $n-i$ rami di $f$, passanti per $O^{\prime}$, e tangenti al piano $b^{\prime} c^{\prime}$, hanno nel punto infinitamente vicino ad $O^{\prime}$, un contatto $(n-i)$ - punto.

Ma per conoscere quale sia la singolarità posseduta nel punto $O^{\prime}$ dalla curva $f^{\prime}$ si faccia la proiezione stereografica del cono quadrico $\Gamma$, scegliendo Annal di Matematica, tomo XXV. 
come centro il punto $A$ e come quadro an piano qualunque $V_{1}$. La generatrice $A O$ di $\Gamma$ incontra il piano $V_{t}$ in un punto $P_{4}$, il quale è l'imagine di ogni punto della generatrice stessa. Inoltre il piano tangente lungo questa al cono $\Gamma$ sega il quadro secondo una retta $p_{1}$, passante per $P_{1}$, ogni punto della quale è l'imagine di un punto di $\Gamma$ infinitamente vicino al centro di proiezione $A$. Le rette del quadro $V_{1}$ passanti per il punto fondamentale $P_{3}$, rappresentano le generatrici di $\Gamma$; tutte le altre rappresentano le sezioni di $\Gamma$ fatte con piani condotti per $A$. Le proiezioni $f_{1}$ della curva $f$, è una curva dell'ordine $2 n-i$, la quale possiede in $P_{1}$ un punto $(n-i)^{-p l o}$ e gli $n-i$ rami di essa passanti per questo punto toccano tutti la retta $p_{1}$, con la quale hanno in comune, oltre $P_{i}$, gli stessi $n-i$ punti infinitamente vicini a $P_{1}$, in modo che nel punto della retta $p_{4}$ infinitamente vicino a $P_{1}$ vengono ad avere un contatto $(n-i)$ - punto $(*)$. La medesima curva $f_{1}$ incontra, fuori di $P_{1}$, la retta $p_{1}$ in $i$ punti distinti fra loro e non passa per il punto $S_{1}$, intersezione della retta $s$ col quadro.

Per mezzo del cono $\Gamma$ resta stabilita fra i punti dei due piani $V^{\prime}$ e $V_{i}$ una corrispondenza birazionale.

Ad un piano di $\Sigma$ passante per il punto $A$ corrisponde in $\Sigma^{\prime}(8$, III) una quadrica $\Phi_{1}$ tangente in $O^{\prime}$ al piano $r^{\prime}$ e contenenti le rette $a_{s}^{\prime} d^{\prime}, s^{\prime}$. Questa quadrioa viene tagliata dal piano $V^{\prime}$ secondo una conica, la quale passa per il punto $S^{\prime}$ in cui il piano stesso incontra la retta $s^{\prime}$ e tocea nel punto $O^{\prime}$ la retta $v^{\prime}$ d'intersezione del piano $V^{\prime}$ col piano ${ }^{\prime}$. La conica cosi ottenuta corrisponde sul piano $V^{\prime}$ a quella secondo cui il piano dato sega il cono $\Gamma$ la quale ha per imagine sul piano $V_{i}$ una retta. Dunque:

1. Alle rette del piano $V_{1}$ corrispondono sul piano $V^{\prime}$ le coniche passanti per il punto $S^{\prime}$, tangenti in $O^{\prime}$ alla retta $v^{\prime}$.

Ad una retta del piano $V^{\prime}$ corrisponde sul cono $T(7$, III) una cubica gobba, la quale passa per i due punti $A$ ed $O$ e tocea in quest'ultima la retta $v$ secondo la qualo il cono $\Gamma$ viene tagliato oltre le rette $a, b, c$, dal cono $C_{0}$; si appoggia poi in un punto, fuori di $A$, alla retta $s$. Questa cubica viene proiettata da $A$ sopra il quadro $V_{i}$ secondo una conica, la quale passa per $i$ due punti $S_{4}$ e $P_{1}$ e tocoa in quest' ultimo la retta $v_{4}$ proiezione della generatrice $v$ del cono $\Gamma$. Dunque:

2. Alle rette del piano $V^{\prime}$ corrispondono sul piano $V_{1}$ le coniche passanti per il punto $S_{i}$ tangenti in $P_{1}$ alla retta $v_{i}$.

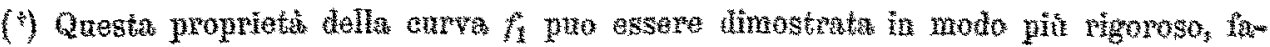
eento nso delle formole della proitzitone stepeografea. 
d' una superficie algebrica per mezzo di trasform. biraz. dello spazio. 115

Così la corrispondenza birazionale fra $\mathrm{i}$ punti dei due piani $V^{\prime}$ e $V_{1}$ è una trasformazione quadratica, per la quale i punti fondamentali sul piano $V^{\prime}$ sono i punti $S^{\prime}, O^{\prime}$ e il punto a questo infinitamente vicino sulla retta $v$; e sul piano $V_{1}$, i punti $S_{1}, P_{1}$ e il punto a questo infinitamente vicino sulla retta $v_{1}$.

La curva $f_{1}$ corrisponde in questa trasformazione alla curva $f^{\prime}$. Si ha perciò :

I. “ Un piano condotto ad arbitrio per il punto $O^{\prime}$ taglia la super" ficie $F^{\prime}$ secondo una curva $f^{\prime}$ dell' ordine $3 n-i$, la quale possiede in $S^{\prime}$ " un punto $n^{-p l o}$ ordinario ed in $O^{\prime}$ un punto $(2 n-i)^{-p l o}$ in cui $n$ delle tan" genti coincidono nella rette d'intersezione del piano considerato col piano $r^{\prime}$, " e le rimanenti $n-i$ nella retta d'intersezione dello stesso piano col piano $b^{\prime} c^{\prime}$. "Eseguendo una trasformazione quadratica per la quale siano fondamentali " sopra il piano dato i punti $S^{\prime}, O^{\prime}$ e il punto a questo infinitamente vicino " sulla prima delle due tangenti in $O^{\prime}$ alla curva $f^{\prime}$, questa si cambia in una " curva $f_{1}$ dell'ordine $2 n-i$ dotata di un punto $(n-i)^{-p t o}$, tale che gli $n-i$ " rami che vi passano toccano ivi una medesima retta sulla quale hanno in " comune gli stessi $n-i$ punti infinitamente vicini a quello. La curva $f_{1}$ si " cambia con una seconda trasformazione quadratica, in un'altra curva $f_{2}$ "fornita di sole singolarità ordinarie."

Suppongasi in particolare che il piano $V^{\prime}$ passi per la retta $d^{\prime}$. Il cono $\Gamma$ che gli corrisponde in $\Sigma$ contiene la retta $d$, e quindi la curva $f$ secondo la quale esso incontra la superficie $F$ gode di tutte le proprietà sopra notate per una curva generale $f$, e di più si appoggia in $n$ punti distinti alla retta $d$. Parimenti la sua proiezione $f_{1}$ sul quadro $V_{1}$ possiede le medesime proprietà dianze trovate, e di più incontra in $n$ punti distinti la retta $d_{3}$, proiezione di $d$.

La quadrica $\Phi^{\prime}$ di $\Sigma^{\prime}$ corrispondente ad un piano di $\Sigma$ condotto per il punto $A$, contiene la retta $d^{\prime}$, epperò viene tagliata dal piano passante per la medesima retta, secondo un'altra retta. Quindi nel caso attuale, la corrispondenza birazionale che ha luogo fra $i$ punti dei due piani $V^{\prime}$ e $V_{1} \grave{e ̀}$ un' omografia. In questa omografia alla retta $d_{i}$ di $V_{t}$ corrisponde la retta $d^{\prime}$ di $V^{\prime}$; e poichè la curva $f_{1}$ incontra quella retta in $n$ punti distinti, come si è testè osservato, così anche la curva $f^{\prime}$ incontra questa in $n$ punti distinti, il che costituisce una proprietà trovata per altra via lungo il corso della dimostrazione del teorema IV del $\mathrm{n}^{\circ}$ 20. Inoltre le curve $f_{\mathrm{s}}$ ed $f^{\prime}$ essendo corrispondenti nell'anzidetta omografia, posseggono le medesime singo- 
larità. Quindi osservando che ai rami di $f^{\prime}$ passanti per $O^{\prime}$ e ivi tangenti al piano $b^{\prime} c^{\prime}$, corrigpondono ai rami di $f_{1}$ passanti per $P_{1}$ e ivi tangenti alla retta $p_{4}$, si conclude :

II. "Un piano condotto ad arbitrio per la retta $d$ taglia ulterior" mente la superficie $F^{\prime}$ secondo una curva $f^{\prime}$ dell' ordine $2 n-i$, la quale "possiede in $O^{\prime}$ un punto $(n-i)$-plo e $i$ suoi $n-i$ rami passanti per questo " punto toceano tutti la retta secondo la quale il piano considerato incontra "il piano $b^{\prime} c^{\prime}$ e hanno in comune con questa gli stessi $n-i$ punti infinita" mente vicini ad $O^{\prime}$."

Si supponga in secondo luogo che il piano $V^{\prime}$ passi per la retta $e^{\prime}$. Il cono $\Gamma$ che gli corrisponde in $\Sigma$ tocea lungo $a$ il piano $t$ e quindi la curva $f$, come la sua proiezione $f_{1}$, su $V_{3}$, non presenta particolarità. Si noti però che nel caso attuale il punto fondamentale $S^{\prime}$ del piano $V^{\prime}$ coincide col punto $E^{\prime}$. Inoltre ad una retta di $\Sigma^{\prime}$ appoggiata alla retta $e^{\prime}$ corrisponde in $\Sigma(11, \mathrm{~V})$ una conica appoggiata alle rette $a, b, c$ ed $s$ e tangente nel punto d'appoggio con $a$ al piano $t$. Quindi la proiezione di questa conica fatta da $A$ sul piano $V_{1}$ passa per i punti $S_{1}$ e $P_{1}$ e tocca in quest' ultimo la retta $t_{1}$, alla quale sono tangenti gli $n-i$ rami di $f_{1}$ passanti per $P_{1}$. La retta $t_{1}$ incontra la curva $f_{1}$, fuori di $P_{1}$ in $i$ punti distinti; percio la curva $f^{\prime}$ possiede in $E^{\prime}$ un punto $i$-plo ordinario, il che è in accordo col teorema $\mathrm{V}$ del $\mathrm{n}^{\circ}$ 23. Dunque:

III. "Un piano condotto ad arbitrio per la retta $e^{\prime}$ taglia la su"perficie $F^{\prime}$ secondo una curva $f^{\prime}$ dell'ordine $2 n$, la quale possiede in $E^{\prime}$ " un punto $i^{-p l o}$ ordinario ed in $O^{\prime}$ un punto $n$-pto in cui le tangenti coin" eidono tutte nella retta d'intersezione del piano considerato col piano $r$. "Eseguendo una trasformazione quadratica come già si è fatto nel precedente " teorema $I$, si perviene ai medesimi risultati là ottenuti. "

Infine il piano $V^{\prime}$ passa per la retta $a_{1}$. Il cono che gli corrisponde in $\Sigma$ tocca lungo $a$ il piano $O s$, e quindi, come si è già trovato innanzi (24), la curva $f$ possiede in $A$ ancora un punto $i$-plo; ma $j$ delle tangenti in questo punto coincidono con la retta $s$. Quindi la curva $f_{1}$ possiede oltre la solita singolarità in $P_{1}$, anohe un punto $j$-plo in $S_{1}$.

Il punto $S_{1}$ giace sulla retta $p_{1}$, e le coniche di $V_{1}$ corrispondenti alle rette di $V^{\prime}$ passano per $S_{1}$ e toccano in $P_{1}$ una retta $v_{1}$, distinta da $p_{1}$. Le coniche di $V^{\prime}$ corrispondenti alle rette di $V_{i}$ passano per $S^{\prime}$ e toccano in $O^{\prime}$ la retta $a_{1}$. Questa retta corrisponde in $V^{\prime}$ al punto fondamentale $S_{1}$ di $V_{i}$; e siccome la curva $f$, possiede in $V_{1}$ un punto $j-p t a$, còs̀ la eurva $f^{\prime}$ incontra 
d' ${ }^{\prime}$ ma superficie algebrica per mezzo di trasform. biraz. dello spazio. 117

la retta $a_{1}$ in $j$ punti fuori di $O^{\prime}$. In tal modo si ritrova la proprietà contenuta nel teorema IV del $n .^{\circ}$ precedente. Inoltre si ha:

IV. " Un piano condotto ad arbitrio per la retta $a_{1}$ taglia ulterior" mente la superficie $F$ " secondo una curva $f$ ' dell'ordine $3 n-i-j$, la quale "possiede in $S^{\prime}$ un punto $n^{-p l o}$ ordinario e in $O^{\prime}$ un punto $(2 n-i-j)^{-p l o}$, " in cui le tangenti coincidono tutte con la retta $a_{1}$. Eseguendo una trasfor" mazione quadratica, la quale abbia come fondamentali sul piano dato $\mathrm{i}$ "punti $S^{\prime}, O^{\prime}$ e il punto a questo infinitamente vicino sulla retta $a_{1}$, la " curva $f^{\prime}$ si cambia in un'altra $f_{1}$, che possiede in $S_{1}$ un punto $j^{-p l o}$ (di " natura incognita) e in $P_{1}$ un punto $(n-i)^{\text {-plo }}$ tale che tutti gli $n-i$ rami "passanti per esso toccano ivi la retta $S_{1} P_{1}$ e hanno in comune con questa "gli stessi $n-i$ punti infinitamente vicini a $P_{1}$."

Con la proprietà contenuta in questo teorema lo studio della superficie $F^{\prime}$ è compiuto, almeno per lo scopo che qui si ha in mira. $\mathbf{E}$ se la retta $a_{1}$ risulta per $F^{\prime}$ una retta $j$-pla ordinaria, la singolarità posseduta nel punto $A$ dalla superficie data $F$ resta in tal modo risoluta. Altrimenti è necessario eseguire su questa superficie la trasformazione studiata nel paragrafo primo.

\section{$\S 5$.}

26. Fra lo spazio $\Sigma^{\prime}$ e un terzo spazio $\Sigma^{\prime \prime}$ si stabilisca la trasformazione del paragrafo primo, assumendo come elementi fondamentali di $\Sigma^{\prime}$ il punto $O^{\prime}$, la retta $a_{1}$, altre due rette $b_{1}$ e $c_{1}$ condotte ad arbitrio per $O^{\prime}$ e la retta $s^{\prime}$, e come elementi di $\Sigma^{\prime \prime}$ un punto qualunque $0^{\prime \prime}$, tre rette $a^{\prime}{ }_{1}, b^{\prime}{ }_{t}, c^{\prime}$, passanti per $O^{\prime \prime}$ e non situate sopra uno stesso piano e una retta qualsiasi $s^{\prime \prime}$. Nella trasformazione cubica cost individuata sono poi altri elementi fonda-

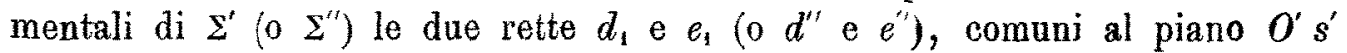

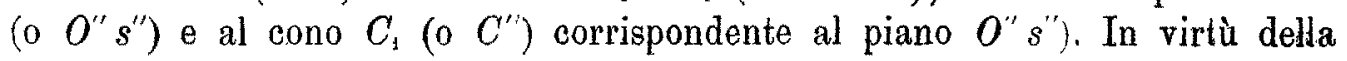
trasformazione stessa alla superficie $F^{\prime}$ di $\Sigma^{\prime}$ corrisponde in $\Sigma^{\prime \prime}$ una nuova superficie, che si indicherà con $F^{\prime \prime}$.

27. Ad una retta $R^{\prime \prime}$ di $\Sigma^{\prime \prime}$ corrisponde in $\Sigma^{\prime}(3$, IV) una cubica gobba $S_{1}$, la quale passa per il punto $O^{\prime}$ e tocea ivi il cono $C_{1}$, si appoggia in due punti alla retta $s^{\prime}$, e in un punto, oltre $O^{\prime}$, a ciascuna delle rette $a_{1}, b_{1}, c_{t}$ e in nessun punto alle rette $d_{1}$ ed $e_{1}$. Quindi ricordando che la superficie $F^{\prime}$ è dell'ordine $3 n-i$ ed ha in $O^{\prime}$ un punto multiplo secondo $2 n-i$ $(18, \mathrm{I})$, che possiede $s^{\prime}$ come retta $n^{-p l a}(22, \mathrm{I})$ e $a_{1}$ come retta $j^{-p l a}(24, \mathrm{II})$, 
ed osservando che non contiene le altre rette fondamentali di $\Sigma^{\prime}$, si trova che essa viene incontrata dalla curva $S_{1}$ in

$$
3(3 n-i)-[(2 n-i)+2 n+j]=5 n-2 i-j,
$$

punti variabili; epperò :

I. "La superficie $F^{\prime \prime}$ è dell'ordine $5 n-2 i-j$."

Poichè ad una retta di $\Sigma^{\prime \prime}$ condotta per il punto $O^{\prime \prime}$ corrisponde in $\Sigma^{\prime}$ $\left(3\right.$, II) una retta passante per il punto $O^{\prime}$, si ha ancora :

II. " La superficie $F^{\prime \prime}$ possiede in $O^{\prime \prime}$ un punto multiplo secondo " $4 n-2 i-j . "$

Si vedrà in seguito $\left(36\right.$, III) quale sia il cono tangente ad $F^{\prime \prime}$ in questo punto $O^{\prime \prime}$.

28. Ad una retta di $\Sigma^{\prime \prime}$ appoggiata in un punto $P^{\prime \prime}$ alla retta $a^{\prime}$, corrisponde in $\Sigma(5, \mathrm{~V})$ una conica, la quale passa per il punto $O^{\prime}$ e tocea ivi il cono $C_{1}$, si appoggia in un punto, oltre $O^{\prime}$, alla retta $a_{1} \mathrm{e}$ in un punto alla retta $s^{\prime}$. Quindi essa incontra la superficie $F^{\prime \prime}$ in

$$
2(3 n-i)-[(2 n-i)+n+j]=3 n-i-j,
$$

punti variabili. Dunque :

I. "La superficie $F^{\prime \prime}$ possiede la retta fondamentale $a_{1}^{\prime}$ come retta " $(2 n-i)-p l a . "$

Ad un punto $P^{\prime \prime}$ della retta $a_{1}^{\prime}$ corrisponde in $\Sigma^{\prime}(4, \mathrm{VI})$ una retta del piano $b_{1} c_{1}$ appoggiata in un punto $M^{\prime}$ alla retta $s^{\prime}$. Poichè questa è $n^{-p l a}$ per la superficie $F^{\prime}(22, \mathrm{I})$, cos̀ quella incontra la superficie stessa in $2 n-i$ punti variakili $e$ in generale tutti distinti fra loro, i quali determinano $2 n-i$ piani passanti per $a_{1}$, cui corrispondono in $\Sigma^{\prime \prime}$ altrettanti piani passanti per $\alpha^{\prime}$. Ripetendo i medesimi ragionamenti fatti per provare il teorema III del n. ${ }^{\circ} 18$, si dimostra che questi piani toccano in $P^{\prime \prime}$ la superficie $F^{\prime \prime}$, e quindi si ha:

II. "I $2 n-i$ piani tangenti alla superficie $F$ " in un punto gene"rico $P^{\prime \prime}$ della retta $a_{1}^{\prime}$ sono tutti distinti fra loro e variabili col punto $P^{\prime \prime}$."

Il piano $b_{1} c_{1}$ taglia la superficie $F^{\prime}$ secondo una curva, la quale possiede in $M^{\prime}$ un punto $n^{-p l o}$ ordinario ed in $O^{\prime}$ una determinata singolarità $(25, \mathrm{I})$. A ciascuna tangente condotta a questa curva dal punto $\boldsymbol{M}^{\prime}$ corrisponde un punto cuspidale della curva $a_{1}^{\prime}$ (Cfr. n. ${ }^{\circ} 18$, IV). Quindi :

III. "Sulla retta $a_{1}^{\prime}$ esiste un numero determinato di punti cu"spidali." 
d'una superficie algebrica per mezzo di trasform. biraz. dello spazio. 119

Ad un piano $\alpha^{\prime \prime}$ di $\Sigma^{\prime \prime}$ condotto ad arbitrio per la retta $a_{\text {, corrisponde }}$ in $\Sigma^{\prime}(5, \nabla \mathrm{I})$ un piano $\alpha_{1}$ passante per la retta $a_{1}$ e fra $\mathrm{i}$ punti di questi due piani ha luogo una determinata trasformazione quadratica, nella quale alla retta $a_{1}^{\prime}$ di $\alpha_{1}^{\prime \prime}$ corrisponde in $\alpha_{1}$ la retta secondo cui questo piano incontra il piano $b_{1} c_{1}$. Ora la curva d'intersezione $f_{1}$ di $\alpha_{1}$ con la superficie $F^{\prime}$, incontra l'anzidetta retta in $n$ punti, fuori di $O^{\prime}$, che sono i punti d'incontro di $F^{\prime}$ con la retta medesima, e sono in generale tutti distinti fra loro. Quindi sono tali anche i punti in cui la retta $a_{1}^{\prime}$ viene segata, fuori di $O^{\prime}$, dalla curva secondo la quale il piano $\alpha^{\prime \prime}$ taglia, oltre $a_{1}^{\prime}$, la superficie $F^{\prime \prime}$. Dunque:

IV. " Un piano condotto ad arbitrio per la retta $a^{\prime}$, taglia ulterior"mente la superficie $F^{\prime \prime}$ in una curva, la quale incontra fuori di $O^{\prime \prime}$, la "retta $a^{\prime}$, in $n$ punti distinti fra loro e variabili col piano considerato. n

Il piano $b_{1} c_{1}$ incontra la superficie $F$ " secondo una curva, che possiede in $O^{\prime}$ un determinato punto multiplo. Ad ogni tangente condotta da $O^{\prime}$ a questa curva corrisponde un piano stazionario della superficie $F^{\prime \prime}$ passante per la retta $a_{1}^{\prime}$. Quindi : " zionari."

V. "Per la retta $a_{1}^{\prime}$ passa un numero determinato di piani sta-

29. Ad una retta di $\Sigma^{\prime \prime}$ appoggiata in un punto $P^{\prime \prime}$ alla retta $b_{1}^{\prime}$ corrisponde in $\Sigma^{\prime}(5, \mathrm{~V})$ una conica, la quale passa per il punto $O^{\prime}$ e tocea ivi il cono $C_{1}$, si appoggia in un punto, oltre $O$, alla retta $b_{1}$ e in un punto alla retta $s^{\prime}$. Quindi essa incontra la superficie $F^{\prime}$ in

$$
2(3 n-i)-[(2 n-i)+n]=3 n-i,
$$

punti variabili; epperò :

I. "La superficie $F^{\prime \prime}$ possiede le rette fondamentali $b_{\mathfrak{t}}^{\prime}$ e $c^{\prime}{ }_{1}^{\prime}$ come "rette multiple secondo $2 n-i-j$."

Ad un punto $P^{\prime \prime}$ di $b_{3}^{\prime}$ corrisponde in $\Sigma^{\prime}(4, \mathrm{VI})$ una retta del piano $c_{1} a_{1}$, appoggiata in un punto $M^{\prime}$ alla retta $s^{\prime}$. Siccome le rette $s^{\prime}$ ed $a_{4}$ sono multiple per $F^{\prime \prime}$ secondo i numeri $n$ e $j$ rispettivamente, così la retta che corrisponde al punto $P^{\prime \prime}$ incontra la superficie $F^{\prime \prime}$ in $2 n-i-j$ punti variabili e in generale distinti fra loro, i quali determinano altrettanti piani passanti per la retta $b_{1}$, i cui corrispondenti piani di $\Sigma^{\prime \prime}$ toccano nel punto $P^{\prime \prime}$ la superficie $F^{\prime \prime}$. Dunque:

II. "I $2-i-j$ piani tangenti alla superficie $F^{\prime \prime}$ in un punto ge" nerico $P^{\prime \prime}$ della retta $b_{1}^{\prime}$, o $c^{\prime}{ }_{4}$ sono tutti distinti fra loro e variabili col "punto $P$ "," 
Il piano $c_{1} a_{1}$ taglia la superficie $F^{\prime \prime}$ secondo una curva, alle tangenti della quale condotte dal punto $M^{\prime}$ corrispondono i punti cuspidali della retta $b_{1}^{\prime}$; epperò :

III. "Sopra ciascuna delle due rette $b_{1}^{\prime}$ e $c^{\prime}{ }_{1}$ esiste un numero de" terminato di punti cuspidali."

Tenendo presente il teorema VI del $n,{ }^{0} 5$, come il teorema IV del n. ${ }^{\circ}$ precedente, si dimostra:

IV. "Un piano condotto ad arbitrio per la retta $b_{t}^{\prime}, o c_{1}^{\prime}$, taglia ul" teriormente la superficie $F^{\prime \prime}$ in una curva la quale incontra, fuori di $O^{\prime}$, la " retta $b_{1}^{\prime}, c_{1}^{\prime}$, in $n$ punti distinti fra loro e variabili col piano considerato."

I piani stazionari della superficie $F^{\prime \prime}$ passanti per la retta $b_{1}^{\prime}$ corrispondono alle tangenti condotte dal punto $O^{\prime}$ alla curva d'intersezione del piano $c_{1} a_{1}$ con la superficie $F^{\prime}$; e quindi :

V. "Per ciascuna delle rette $b_{1}^{\prime} c^{\prime}{ }_{1}$ passa un numero determinato "di piani stazionari."

30. Alle rette $a^{\prime}, b^{\prime}, c^{\prime}$ della stella $\left(O^{\prime}\right)$ corrispondono nella stella $\left(O^{\prime \prime}\right)$ tre rette $a^{\prime \prime}, b^{\prime \prime}, c^{\prime \prime}$. Poichè quelle sono multiple per la superficie $F^{\prime}$ secondo i numeri $n(18, \mathrm{II}), n-i$ e $n-i(19, \mathrm{I})$ rispettivamente e non sono fondamentali per lo spazio $\Sigma$, così queste hanno eguali multiplicità per la superficie $F^{\prime \prime}$. Dunque :

I. "La superficie $F^{\prime \prime}$ passiede le rette $a^{\prime \prime}, b^{\prime \prime}, c^{\prime \prime}$ come rette mul" tiple secondo i numeri $n, n-i, n-i$ rispettivamente."

Ad una retta di $\Sigma^{\prime \prime}$ appoggiata alla retta $d_{1}^{\prime}$ corrisponde in $\Sigma^{\prime}(5$, VII) una conica, la quale si appoggia in un punto a ciascuna delle rette $a_{1}, b$, $c_{1}, d_{1}, s_{1}$. Quindi essa incontra la superficie $F^{\prime}$ in

$$
2(3 n-i)-(j+n)=5 n-2 i-j .
$$

punti variabili; epperò :

II. "La superficie $F^{\prime \prime}$ non contiene le rette $d^{\prime}{ }_{1}$ ed $e^{\prime}{ }_{1}$ " "

31. Ad una retta di $\Sigma^{\prime \prime}$ appoggiata in un punto $P^{\prime \prime}$ alla retta $s^{\prime \prime}$ corrisponde in $\Sigma^{\prime}\left(5\right.$, II) una conica appoggiata in due punti alla retta $s^{\prime}$ e in un punto a ciascuna delle rette $a_{1}, b_{1}, c_{1}$. Quindi essa incontra la superficie $F^{\prime \prime}$ in

$$
2(3 n-i)-(2 n+j)=4 n-2 i-j,
$$

punti variabili; epperò :

I. "La superficie $F^{\prime \prime}$ possiede la retta fondamentale $s^{\text {" come }}$ " retta $n$-pla." 
Ad un punto $P^{\prime \prime}$ di $s^{\prime \prime}$ corrisponde in $\Sigma^{\prime}(4, V)$ una generatrice del cono $C_{1}$. Quindi come i teoremi II e III del n. ${ }^{\circ} 22$, così si hanno i seguenti: II. " Gli $n$ piani tangenti alla superficie $F^{\prime \prime}$ in un punto generico $P^{\prime \prime}$

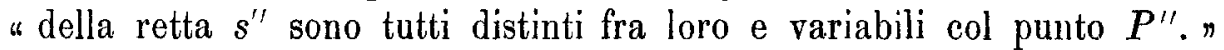

III. "Sulla retta $s^{\prime \prime}$ esistono tanti punti cuspidali quante sono le " generatrici del cono $C_{1}$ tangenti alla superficie $F^{\prime}$."

Tenendo presente il teorema III del n. ${ }^{\circ} 5$, come i teoremi IV e V del n. 22 , si dimostrano gli altri:

IV. "Un piano condotto ad arbitrio per la retta $s^{\prime \prime}$ taglia ulterior" mente la superficie secondo una curva che incontra la retta medesima $s^{\prime \prime}$ " in punti tutti fra loro distinti e variabili col piano considerato."

V. "Per la retta $s$ " passano tanti piani stazionari quante sono le "sezioni fatte nella superficie $F^{\prime}$ con piani condotti per la retta $s^{\prime}$, le quali "riescono tangenti al cono $C_{1}$."

32. Altre proprietà della superficie $F^{\prime \prime}$ si dimostrano più facilmente ricorrendo alla trasformazione birazionale che in virtì delle trasformazioni stabilite fra gli spazi $\Sigma$ e $\Sigma^{\prime}$, e $\Sigma^{\prime}$ e $\Sigma^{\prime \prime}$, nasce fra $\Sigma$ e $\Sigma^{\prime \prime}$.

Si ricordi dapprima che ad una retta $R^{\prime \prime}$ di $\Sigma^{\prime \prime}$ corrisponde in $\Sigma(15$, IV) una curva del $5 .^{\circ}$ ordine $s_{0}$, la quale possiede in $A$ un punto doppio in cui una delle tangenti è la retta $s$. Questa curva deve incontrare la superficie data $F$ in $5 n-2 i-j$ punti variabili, perchè in tanti punti variabili la retta $R^{\prime \prime}$ incontra $(27, \mathrm{I})$ la superficie $F^{\prime \prime}$. Quindi dicendo $x$ il numero de'suoi punti d'intersezione con $F$, riuniti in $A$, si ha

$$
5 n-x=5 n-2 i-j
$$

donde

$$
x=2 i+j \text {. }
$$

Ora ammesso che il numero dei punti d'intersezione runiti in $A$ della superficie $F$ con una curva la quale possegga in $A$ la medesima singolarità di cui è ivi dotata la curva $S_{0}$, non dipenda dall' ordine della curva, ma soltanto dal suo modo di comportarsi nelle immediate vicinanze del punto $A$, si può conoludere :

I. "Se una curva qualunque possiede in $A$ un punto doppio ed una " delle tangenti in questo punto è la retta $s$, il numero dei punti d'interse" zione, riuniti in $A$, della curva data con la superficie $F$ è $2 i+j$."

La superficie $\varphi_{0}$ di $\Sigma$ corrispondente $\left(15\right.$, III) ad un piano $U^{\prime \prime}$ di $\Sigma^{\prime \prime}$ taglia la superficie data $F$ secondo una curva $f_{0}$ dell'ordine $5 n$. La superficie $\varphi_{0}$ 
contiene la retta $g$, la quale incontra la superficie $F$ in $n$ punti $H_{1}, H_{2}$, $H_{3}, \ldots, H_{n}$, tutti distinti fra loro; quindi la curva $f_{0}$ si appoggia alla retta $g$ in questi punti. La superfieie $\varphi_{3}$ possiede come doppia la retta a e lungo questa è toccata dai medesimi piani tangenti fissi $O s$ e $k$; inoltre, quella delle sue falde tangente lungo $a$ al piano $O s$ ha in comune, oltre $a$, con la falda del cono $C_{0}$ che lungo la medesima retta tocca lo stesso piano, tre rette infinitamente vicine ad $\alpha$. Quindi la curva fo possiede un punto doppio in ciascuno degli $n-i$ punti $K_{1}, K_{2}, K_{3}, \ldots, K_{n-i}$, tutti distinti fra loro, nei quali la superficie $F$ incontra, fuori di $A$, la retta $a$, e dei due rami passanti per esso uno tocca il piano $k$ e l'altro ha in comune con la falda del cono $C_{0}$ tangenti lungo $a$ al piano $O s$, tre punti infinitamente vicini al punto doppio che si considera, oltre i punti riuniti nel punto doppio stesso. La superficie $\varphi_{0}$ possiede come doppie le rette $b$ e $c$, ciascuna delle quali incontra la superficie $F$ in $n$ punti distinti; e questi sono altrettanti punti doppi per la curva $f_{0}$. Infine la superficie $\varphi_{3}$ possiede in $A$ un punto triplo, in cui il cono tangente si scinde in un cono quadrico e nel piano $O s$ e la falda della superficie stessa tangente in $A$ a questo piano oscula la falda del cono $C_{0}$ che lungo la retta $a$ tocea il medesimo piano $O s$. La superficie data $F$ possiede in $A$ un punto $i-p l o$, in cui il cono tangente $\Delta$ ha come $j$-plo la retta $s$. Quindi la curva $f_{0}$ possiede in $A$ un punto multiplo secondo $3 i$, e delle $i$ tangenti in questo punto ottenute tagliando il cono $\Delta$ col piano $O s, j$ coincidono con la retta $s$ e le rimanenti $i-j$ sono tutte distinte fra loro e $\mathrm{i}$ rami di $f_{0}$ tangenti a queste rette osculano in $A$ il cono $C_{0}$. Riepilogando si ha dunque:

II. "La curva $f_{0}$ secondo la quale una superficie qualunque $\varphi_{0}$ taglia " la superficie data $F$ è dell'ordine $5 n$, si appoggia in $n$ punti distinti $H$ " alla retta $g$, possiede sopra la retta $a, n-i$ punti doppi $K$, tutti distinti "fra loro, e dei due rami passanti per ciascuno di questi punti uno tocca il "piano $k$ e l'altro ha, fuori del punto medesimo, ma in un punto infinita" mente vicino ad esso, un contatto tripunto con la falda del cono $C_{0}$ tan"gente lungo a al piano $O s$, infine possiede in $A$ un punto multiplo secondo " $3 i$ e de' suoi rami passanti per $A, i-j$ toccano in $A$, secondo direzione " distinte fra loro, il piano $O s$ ed inoltre osculano l'anzidetta falda del "cono $C_{0} . "$

Ad una retta $R^{\prime \prime}$ di $\Sigma^{\prime \prime}$ appoggiata in un punto $P^{\prime \prime}$ alla retta $g^{\prime \prime}$ corrisponde in $\Sigma(16, \mathrm{II})$ una curva del $4 .^{\circ}$ ordine la quale si comporta in $A$, come una curva $S_{0}$; quindi in virtù del precedente teorema $I$, essa incontra la superficie $F$ in $4 n-2 i-j$ punti variabili; epperò : 
d'una superficie algebrica per mezzo di trasform. biraz. dello spazio. 123

\section{III. " La superficie $F^{\prime \prime}$ possiede la retta fondamentale $g$ " come "retta $n^{-p l o} . "$}

La retta considerata $R^{\prime \prime}$ si riguardi come intersezione di un piano qualunque $U^{\prime \prime}$ condotto per essa con il piano $V^{\prime \prime}$ che la proietta da $O^{\prime \prime}$. $\Lambda \mathrm{l}$ primo piano corrisponde in $\Sigma\left(15\right.$, III) una superficie $\varphi_{0} ;$ al secondo $(16, \mathrm{I})$, un cono $\Gamma_{g}$ contenente la retta $g$. La retta $R^{\prime \prime}$ incontra la superficie $F^{\prime \prime}$ (III) in $4 x-2 i-j$ punti variabili, ossia la superficie $H^{\prime \prime}$ e i due piani $U^{\prime \prime}$ e $V^{\prime \prime}$ hanno in comune $4 n-2 i-j$ punti variabili; quindi altrettanti punti comuni variabili debbono avere le due superficie $F$ e $\varphi_{0}$ e il cono $\Gamma_{g}$, ossia la curva d'intersezione $f_{0}$ di quelle due superficie deve incontrare questo cono in $4 n-2 i-j$ punti variabili. Ora (II) la curva $f_{0}$ si appoggia in $n$ punti distinti $H$ alla retta $g$; inoltre $i$ coni $\Gamma_{g}$ formano un faseio; quindi fra questi ve ne sono $n$, ciascuno dei quali tocea la curva $f_{0}$ in uno, ed uno solo, di quei punti $H$. Ognuno di questi $n$ coni incontra dunque la curva $f_{n}$ in $4 n-2 i-j-1$ punti variabili; perciò il piano ad esso corrispondente in $\Sigma^{\prime \prime}$ taglia il piano considerato $U^{\prime \prime}$ secondo una retta $R^{\prime \prime}$ appoggiata in $P^{\prime \prime}$ alla $g^{\prime \prime}$, la quale incontra la superficie $F^{\prime \prime}$ in $4 n-2 i-j-1$ punti variabili. Quindi nel punto $P^{\prime \prime}$ cadono $n+1$ punti d'intersezione della anzidetta retta $R^{\prime \prime}$ con la superficie $F^{\prime}$, epperò la retta medesima tocca in $P^{\prime \prime}$ questa superficie, e quindi il piano delle due rette $R^{\prime \prime}$ e $g^{\prime \prime}$ tocca anche esso in $P^{\prime \prime}$ la superficie $F^{\prime \prime}$. Dunque i piani di $\Sigma^{\prime \prime}$ corrispondenti agli $n$ anzidetti coni $\Gamma_{g}$ di $\Sigma$, toccano in $P^{\prime \prime}$ la superficie $F^{\prime \prime}$; e siccome quei coni sono in generale tatti distinti fra loro, così si ha:

IV. "Gli $n$ piani tangenti alla superficie $F^{\prime \prime}$ in un punto gene-

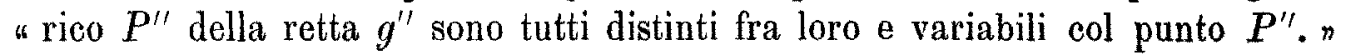

Gli $n$ coni $\Gamma_{g}$ ora considerati tagliano la superficie $\varphi_{0}$ corrispondente al piano $U^{\prime \prime}$ in $n$ eurve del $4 .^{\circ}$ ordine passanti rispettivamente per gli $n$ punti $H$ in oui la retta $g$ incontra la superficie $F$. Le medesime curve corrispondono $\left(16\right.$, II) alle $n$ rette d'intersezione del piano $U^{\prime \prime}$ con i piani tangenti in $P^{\prime \prime}$ alla superficie $F^{\prime \prime}$. Se due di questi piani coineidono, coincidono pure due di quelle curve. Ma perchè gli $n$ punti $H$ sono tutti distinti fra loro, ciò non può accadere; dunque :

V. "Sulla retta $y^{\prime \prime}$ non esistono punti cuspidali."

Con ragionamenti affatto analoghi a quelli seguiti per dimostrare il teorema IV del n. ${ }^{\circ} 20$, si trova:

VI. "Un piano condotto ad arbitrio per la retta $g^{\prime \prime}$ taglia ulterior" mente la superficie $F^{\prime \prime}$ in una curva, che incontra la retta $g^{\prime}$, fuori di $O^{\prime}$, " in $n$ punti distinti e variabili col piano considerato." 
VII. "Per la retta $g$ " non passano piani stazionari."

33. Ad una retta $R^{\prime \prime}$ di $\Sigma^{\prime \prime}$ appoggiata in un punto $P^{\prime \prime}$ alla retta $h^{\prime \prime}$ corrisponde in $2^{\prime \prime}(16, \mathrm{IV})$ una curva del $4 .^{\circ}$ ordine, la quale passa per il punto $A$ e tocca ivi la retta $s$; quindi $(24, I)$ essa incontra la superficie $F$ in $4 n-i-j$ punti variabili; epperò :

I. "La superficie $F^{\prime \prime}$ possiede la retta fondamentale $h^{\prime \prime}$ come " retta $(n-i)-p l o . "$

Si consideri ancora la retta $R^{\prime \prime}$ come intersezione di un piano $U^{\prime \prime}$ condotto ad arbitrio per essa il piano $V^{\prime \prime}$ che la proietta da $O^{\prime}$. A quest'ultimo piano corrisponde in $\Sigma(16$, III $)$ un cono $\Gamma_{h}$ tangente lungo a ai due piani $O s$ e $k$; si dimostra come nel caso precedente, tenendo conto del teorema $\mathrm{I}$, che questo cono incontra la curva $f_{0}$, intersezione di $\varphi_{0}$ con $F$, in $4 n-i-j$ punti variabili. Ora $\left(30\right.$, II) la curva $f_{0}$ possiede sopra la retta $a, n-i$ punti doppi $K$, distinti fra loro, in ciascuno dei quali un ramo di fo tocca il piano $k$, cui è tangente anche ciascuno dei coni $\Gamma_{h}$. Poichè questi coni formano un fascio, fra essi ve ne sono $n-i$, in generale distinti fra loro, ciascuno dei quali oscula in uno, ed un solo, dei punti $k$ il ramo della curva $f_{0}$ passante per il punto stesso e ivi tangente al piano $k$. Questo cono incontra quindi la curva $f_{0}$ in $4 n-i-j-1$ punti variabili; epperò si dimostra come nel caso precedente, che il piano di $\Sigma^{\prime \prime}$ che ad esso corrisponde tocea nel punto $P^{\prime \prime}$ la superficie $F^{\prime \prime}$. Dunque:

II. "Gli $n-i$ piani tangenti alla superficie $F^{\prime \prime}$ in un punto gene-

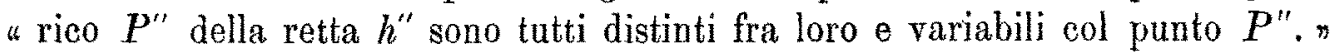

Inoltre come $\mathrm{i}$ teoremi $\mathrm{V}$, VI e VII del $\mathrm{n}^{\circ}$ precedente si dimostrano gli altri :

III. "Sulla retta $h^{\prime \prime}$ non esistono punti cuspidali."

IV. "Un piano condotto ad arbitrio per la retta $h$ " taglia ulterior"mente la superficie $F^{\prime \prime}$ in una curva ehe incontra la retta $h^{\prime}$, fuori di $O^{\prime}$ " in $n-i$ punti distinti e variabili col piano considerato."

V. "Per la retta $h^{\prime \prime}$ non passano piani stazionari."

34. Ad una retta $R^{\prime \prime}$ di $\Sigma^{\prime \prime}$ appoggiata in un punto $P^{\prime \prime}$ alla retta $l^{\prime \prime}$ corrisponde in $\Sigma(16, V D)$ una curva del $4 .^{\circ}$ ordine la quale pussiede in $A$ un punto doppio, in cui una delle tangenti giace sul piano $O s$ od è distinta dalle rette $a$ ed $s$, mentre l'altra è situata fuori di questo piano; quindi essa incontra la superficie $F$ in $4 n-2 i$ punti variabili, epperò:

I. "La superficie $F^{\prime \prime}$ possiede la retta fondamentale $l^{\prime}$ come "retta $(n-j) \cdot p l o$." 
d'una superficie algebrica per mezzo di trasform. biraz. dello spazio. 125

La retta $R^{\prime \prime}$ si consideri sempre come intersezione di un piano $U^{\prime \prime}$ condotto ad arbitrio per essa con il piano $V^{\prime \prime}$ che la proietta da $O^{\prime \prime}$. A quest'ultimo piano corrisponde in $\Sigma(16, V)$ un cono $\Gamma_{l}$, che oscula lungo la retta $a$ con una falda la falda del cono $C_{0}$ tangente lungo la medesima retta al piano $O s$; si dimostra come nei casi precedenti, tenendo conto del teorema $I$, che questo cono incontra la curva $f_{0}$, intersezione di $\varphi_{0}$ con $F$, in $4 n-2 i$ punti variabili. Ora (30, II) la curva fo possiede sopra la retta $a$, $n-i$ punti doppi $K$, tutti distinti fra loro e quello dei due rami di $f_{0}$ passanti per ogni punto $K$ che tocca in $K$ il piano $O s$, ha di comune col cono $C_{0}$ tre punti, oltre $K$ infinitamente vicini fra loro e a $K$; epperò il ramo stesso oscula il cono $\Gamma_{l}$. Fra $i$ coni $\Gamma_{l}$ v'è lo stesso cono $C_{0}$, il quale per la proprietà ora ricordata della curva $f_{0}$, incontra la curva stessa non in $4 n-2 i$ punti variabili, ma in

$$
(4 n-2 i)-(n-i)=3 n-i
$$

punti variabili soltanto. Al cono $C_{0}$ corrisponde in $\Sigma^{\prime \prime}$ il piano $O^{\prime \prime} s^{\prime \prime}$, il quale dunque taglia il piano $U^{\prime \prime}$ in una retta, che deve incontrare la superficie $F^{\prime \prime}$ in $3 n-i$ punti variabili. Quindi nel punto $P^{\prime \prime}$ in cui questa retta si appoggia alla $l^{\prime \prime}$ cadono

$$
(5 n-2 i-j)-(3 n-i)=2 n-i-j,
$$

punti d'intersezione. Ma per il teorema precedente, il punto $P^{\prime \prime}$ è $(n-j)-p l o$ per $F^{\prime \prime}$; dunque la retta anzidetta tocca in $P^{\prime \prime}$ la superficie $F^{\prime \prime}$, ed ba in comune con essa, fuori di $P^{\prime \prime}$, altri

$$
(2 n-i-j)-(n-j)=n-i,
$$

punti infinitamente vicini a $P^{\prime \prime}$. Dunque fra $\mathrm{i}$ piani tangenti alla superficie $F^{\prime \prime}$ in un punto generico della retta $l^{\prime \prime}$ v'è sempre il piano $O^{\prime \prime} s^{\prime \prime}$, ed inoltre questo taglia la superficie stessa, fuori della retta $l^{\prime \prime}$ contata $n-j$ volte, in altre $n-i$ rette infinitamente vicine ad $l^{\prime \prime}$.

La curva $f_{0}$ possiede $i-j$ rami, che passano per il punto $A$ e toccano ivi il piano $O s$ e di più osculano il cono $C_{0}$ e quindi anche il cono $\Gamma_{l}$. Fra i coni $\Gamma_{l}$, formanti un fascio, ve ne sono $i-j$, in generale distinti fra loro, ciascuno dei quali ha in comune con uno degli anzidetti $i-j$ rami di $f_{0}$ passanti per $A$, tre punti, oltre $A$, infinitamente vicini ad $A$. Ognuno di questi coni incontra quindi la curva $f_{0}$ in $4 n-2 i-1$ punti variabili, epperò il piano ad esso corrispondente in $z^{\prime \prime}$ tocea in $P^{\prime \prime}$ la superficie $F^{\prime \prime}$. 
Raccogliendo si ha dunque:

II. "Degli $n-j$ piani tangenti alla superficie $F^{\prime \prime}$ in un punto ge" nerico $P^{\prime \prime}$ della retta $l^{\prime \prime}, i-j$ sono distinti e variabili col punto $P^{\prime \prime}$, e i "rimanenti $n-i$ coincidono col piano $O^{\prime \prime} s^{\prime}$. Questo piano taglia la super" ficie $F$ ", oltre le rette $s^{\prime \prime}, g^{\prime \prime}, h^{\prime \prime}$ ed $l^{\prime \prime}$ contate $n, n, n-i$ e $n-j$ volte "rispettivamente; in altre $n-i$ rette infinitamente vicine ad $l$ "."

Come gli ultimi tre teoremi dei due numeri precedenti, si dimostrano gli altri :

III. "Sulla retta $l^{\prime \prime}$ non esistono punti cuspidali."

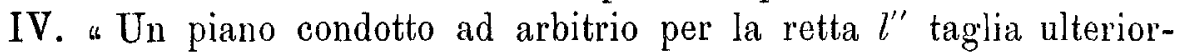
"mente la superficie $F "$ in una curva, che incontra la retta $l$ ", fuori di $O^{\prime \prime}$, "in $i-j$ punti distinti e variabili col piano considerato."

V. "Per la retta $l$ " non passano piani stazionari."

35. La retta $g^{\prime \prime}$ si appoggia alla retta $s^{\prime \prime}$ in un punto $G^{\prime \prime}$. Una retta $R^{\prime \prime}$ condotta ad arbitrio per $G^{\prime \prime}$ determina un piano $\sigma^{\prime \prime}$ passante per $s^{\prime \prime}$, cui corrisponde in $\Sigma^{\prime}$ un piano $\sigma^{\prime}$ passante per $s^{\prime}$, e fra i punti di questi due piani ha luogo una trasformazione quadratica, in virtù della quale alla retta $R^{\prime \prime}$ di $\sigma^{\prime \prime}$ corrisponde in $\sigma^{\prime}$ una conica, la quale passa per $\mathrm{i}$ punti $A_{1}, B_{1}, C_{1}$, e $G_{1}$, in cui questo piano incontra le rette $a_{1}, b_{1}, c_{1}$ e $g_{1}$, essendo $y_{1}$ la retta di $\Sigma^{\prime}$ corrispondente alle rette $g^{\prime \prime}$ di $\Sigma^{\prime \prime}$, ossia l'intersezione, oltre $a_{1}$, del piano $r$ col cono $C_{1}$. Delle quattro rette anzidette soltanto la prima, $a_{1}$, appartiene alla superficie $F^{\prime}$, ed è $j$-pla per essa $(24$, II); quindi la conica, corrispondente alla retta $R^{\prime \prime}$, la qual conica incontra in due punti la retta $s^{\prime}$, $n^{-p l a}$ per $F^{\prime}$, interseca questa superficie in

$$
2(3 n-i)-(2 n+j)=4 n-2 i-j,
$$

punti variabili, epperò :

I. "La superficie $F^{\prime \prime}$ possiede in $G^{\prime \prime}$ un punto $n^{-p l o . " ~}$

Il piano $r^{\prime}$ taglia la superficie $F^{\prime}(24$, III) secondo rette soltanto, le quali passano tutte per il punto $O^{\prime}$. Quindi la retta $g$ situata sopra questo piano e passante per $O^{\prime}$, non incontra la superficie $F^{\prime}$ fuori di questo punto. Perciò fra le coniche di $\Sigma$, corrispondenti alle rette di $\Sigma^{\prime \prime}$ condotte per il punto $G^{\prime \prime}$, appoggiate alla retta $g_{1}$, non ve $n^{\prime h a}$ alcuna, la quale incontri la superficie $F^{\prime}$ in un punto di questa retta. Quindi fra le rette di $\Sigma^{\prime \prime}$ condotte per il punto $G^{\prime \prime}$ non ve $n^{\prime}$ ha alcuna ehe tocchi in $G^{\prime \prime}$ la superficie $F^{\prime \prime}$, ad eccezione di quelle situate sul piano $O^{\prime \prime} s^{\prime \prime}$, ognuna delle quali incontra la superficie stessa, fuori delle rette $h^{\prime \prime}$ ed $l^{\prime \prime}(33$, I $e 32$, II $)$ in $2 n$ punti riuniti in $G^{\prime \prime}$. Dunque : 
d'una superficie algebrica per mezzo di trasform. biraz. dello spazio. 127

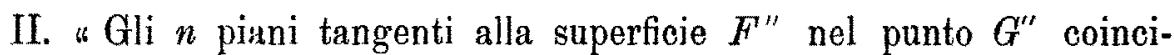
"dono col piano $O$ " $s^{\prime \prime}$ ed ogni retta di questo piano condotta per $G^{\prime \prime}$ ha in " comune con la superficie, $2 n$ punti riuniti in $G^{\prime \prime}$."

La retta $h^{\prime \prime}$ si appoggia alla retta $s^{\prime \prime}$ in un punto $H^{\prime}$. Come nel caso precedente si vede che alle rette di $\Sigma^{\prime \prime}$ condotte per il punto $H^{\prime \prime}$ corrispondono in $\Sigma^{\prime}$ le coniche appoggiate in due punti alla retta $s^{\prime}$ e in un punto a ciascuna delle rette $a_{1}, b_{1}, c_{1}$, ed $h_{1}$, essendo $h_{1}$ la retta di $\Sigma^{\prime}$ corrispondente alla retta $h^{\prime \prime}$, epperò l'intersezione, oltre $a_{1}$, del piano $b^{\prime} c^{\prime}$ col cono $C_{1}$. Quindi come il precedente teorema I, si ha l'altro:

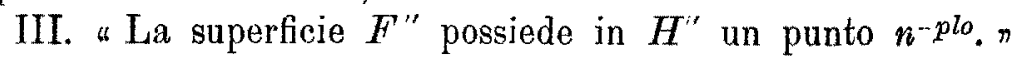

La retta $h_{1}$ incontra la superficie $F^{\prime}(21$, VI) in $i$ punti distinti fra loro, i quali determinano altrettanti piani passanti per $s^{\prime}$, cui corrispondono $i$ piani passanti per $s^{\prime \prime}$, i quali toccano la superficie $F^{\prime \prime}$ nel punto $H^{\prime}$. Inoltre anche il piano $O^{\prime \prime} s^{\prime}$ è tangente in $H^{\prime \prime}$ ad $F^{\prime \prime}$, perchè ogni sua retta condotta per $H^{\prime \prime}$, incontra $F^{\prime \prime}$, fuori delle rette $g^{\prime \prime}$ ed $l^{\prime \prime}$ (30, III e 32 , II), in $2 n-i$ punti riuniti in $H^{\prime \prime}$. Dunque:

IV. "Degli $n$ piani tangenti alla superficie $F^{\prime \prime}$ nel punto $H^{\prime \prime}, i$ sono "distinti fra loro e i rimanenti $n-i$ coincidono col piano $O^{\prime \prime} s$ ", ed ogni

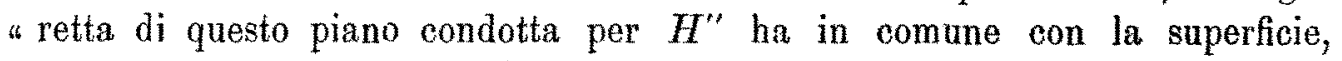
" $2 n-i$ punti riuniti in $H$ "."

La retta $L^{\prime \prime}$ si appoggia alla retta $s^{\prime \prime}$ in un punto $L^{\prime \prime}$. Una retta $R^{\prime \prime}$ di $\Sigma^{\prime \prime}$ determina un piano $\sigma^{\prime \prime}$ passante per $s^{\prime \prime}$, cui corrisponde in $\Sigma^{\prime}$ un piano $\sigma^{\prime}$ passante per $s^{\prime}$. Alla retta $R^{\prime \prime}$ corrisponde su questo piano una conica circoscritta al triangolo $A_{1} B_{1} C_{4}$ e tangente in $A_{4}$ alla retta di $\sigma^{\prime}$ corrispondente nella trasformazione quadratica fra $\sigma^{\prime}$ e $\sigma^{\prime \prime}$, alla retta d'intersezione di $\sigma^{\prime \prime}$ col piano $a^{\prime}, l^{\prime \prime}$ e quindi tangente al piano di $\Sigma^{\prime}$, passunte per $a_{1}$, corrispondente al piano $a_{1}^{\prime} l^{\prime \prime}$. In virtù dell'arbitrarietà degli elementi fondamentali di $\Sigma^{\prime}$ e di $\Sigma^{\prime \prime}$, quest'ultimo piano $a^{\prime}, l^{\prime \prime}$ può essere qualunque, e quindi può sempre supporsi che il suo corrispondente sia tale che tocchi le falde della superficie $F^{\prime}$ passanti per la retta $a_{1}$ soltanto in alcuni punti di questa retta. Quindi dei punti d'incontro di $F^{\prime}$ con la conica sopra considerata, soltanto $i$, almeno in generale, ne cadono nel punto $A_{4}$; altri $2 n$ ne cadono nei due punti d'appoggio della conica stessa con la retta $s^{\prime}$. Dunque:

V. "La superficie $F^{\prime \prime}$ possiede in $L^{\prime \prime}$ un punto $n^{-p l o .}$."

Il piano di $\Sigma^{\prime}$ corrispondente al piano $a_{1}^{\prime} l^{\prime \prime}$ di $\Sigma^{\prime \prime}$, per quanto si è ora osservato e per il teorema IV del n. ${ }^{\circ} 24$, taglia la superficie $F^{\prime}$, oltre la retta $a_{1}$, secondo una corva $f^{\prime}$, la quale incontra $a_{1}$, fuori di $O^{\prime}$, in $j$ punti, 
i quali sono distinti, oppure tutti o solo in parte coincidenti. Ciascuno di questi $j$ punti determina un piano $\sigma^{\prime}$ passante per la retta $s^{\prime}$, al quale corrisponde in $\Sigma^{\prime \prime}$ un piano passante per $s^{\prime \prime}$ e alle rette di questo piano condotte per il punto $L^{\prime \prime}$ corrispondono in quello le coniche circoscritte al triangolo $A_{1} B_{1} C_{1}$ e tangenti in $A_{1}$ alla superficie $F^{\prime}$; perciò le rette anzidette toccano in $L^{\prime \prime}$ la superficie $F^{\prime \prime}$. Dunque ai $j$ punti nei quali la curva $f^{\prime}$ incontra, fuori di $O^{\prime}$, la retta $a_{1}$, corrispondono altrettanti piani passanti per $s^{\prime \prime}$ e tangenti in $L^{\prime \prime}$ alla superficie $F^{\prime \prime}$; e questi piani sono come quei punti, o tutti distinti oppure tutti od in parte coincidenti. Ogni retta del piano $O^{\prime} s^{\prime \prime}$ condotta per il punto $L^{\prime \prime}$ incontra la superficie $F^{\prime \prime}$, fuori di $L^{\prime \prime}$, soltanto nei punti in cui essa interseca le rette $g^{\prime \prime}$ e $h^{\prime \prime}$; quindi $(30$, III e 31 , I) nel punto $L^{\prime \prime}$ cadono $3 n-i-j$ punti d'intersezione; epperò :

VI. "Degli $n$ piani tangenti alla superficie $F^{\prime \prime}$ nel punto $L^{\prime}, j$ sono " distinti dal piano $O^{\prime \prime} s^{\prime \prime}$, e possono fra loro essere o tutti distinti oppure " tutti od in parte coincidenti, e i rimanenti $n-j$ coincidono col piano $O$ " $s^{\prime \prime}$, " ed ogni retta di questo piano condotta per $L^{\prime \prime}$ ha in comune con la super" ficie $3 n-i-j$ punti riuniti in $L " . "$

36. Si passi in fine a studiare le sezioni piane della superficie $F^{\prime \prime}$.

Innanzi tutto ad un piano $z^{\prime \prime}$ di $\Sigma^{\prime \prime}$ condotto per la retta $s^{\prime \prime}$ corrisponde in $\Sigma^{\prime}$, un piano $\sigma^{\prime}$ passante per $s^{\prime}$, e fra i punti di questi due piani ha luogo una trasformazione quadratica, per la quale sono fondamentali sul piano $\sigma^{\prime} i$ punti $A_{1}, B_{1}, C_{1}$ in cui esso incontra le rette $a_{1}, b_{1}, c_{1}$ e sul piano $\sigma^{\prime \prime}$ i punti $A^{\prime}{ }_{1}, B^{\prime}{ }_{1}, C_{1}^{\prime}$, in cui esso incontra le rette $a_{1}^{\prime}, b_{4}^{\prime}, c_{1}^{\prime}(5$, III). La curva d'intersezione $f^{\prime \prime}$ di $\sigma^{\prime \prime}$ con $F^{\prime \prime}$ è la curva di questo piano $\sigma^{\prime \prime}$ corrispondente nell'anzidetta trasformazione alla curva di intersezione $f^{\prime}$ di $\sigma^{\prime}$ con $F^{3}$. Ora (22, IV) quest' ultima curva è dell'ordine $2 n-i$ e possiede in $A_{1}$ un punto multiplo secondo $j$; quindi la curva $f^{\prime \prime}$ è dell'ordine $4 n-2 i-j$ e possiede in $A^{\prime}{ }_{1}, B_{1}^{\prime}, C^{\prime}$, punti multipli ordinari secondo i numeri $2 n-i, 2 n-i-j$ $2 n-i-j$ rispettivamente. Inoltre quella curva possiede punti multipli ordinari secondo $n, n-i, n-i$ nei punti $A^{\prime}, B^{\prime}, C^{\prime}$ in cui il suo piano incontra le rette $a^{\prime}, b^{\prime}, c^{\prime}$; quindi questa possiede punti multipli ordinari degli stessi gradi nei punti $A^{\prime \prime}, B^{\prime \prime}, C^{\prime \prime}$ in cui il suo piano incontra le rette $a^{\prime \prime}, b^{\prime \prime}, c^{\prime \prime}$. Queste proprietà sono d'accordo con quelle dimostrate nei teoremi: I, 31; $\mathrm{I}, 28 ; \mathrm{I}, 29 ; \mathrm{I}, 30$. Infine, poichè la curva $f^{\prime}$ possiede in $A_{1}$ un punto $j$-plo di natura incognita, cosi la curva $f^{\prime \prime}$ incontra la retta fondamentale $B_{1}^{\prime} C^{\prime}{ }_{1}$, fuori dei punti $B_{1}^{\prime}$, e $C_{1}^{\prime}$, in altri $j$ punti, $i$ quali possono coincidere e dar luogo anche ad un punto $j^{-p l o}$ di $f^{\prime \prime}$. Dunque: 
I. "Un piano condotto ad arbitrio per la retta $s$ " taglia ulterior" mente la superficie $F^{\prime \prime}$ secondo una curva $f^{\prime \prime}$ dell' ordine $4 n-2 i-j$, la " quale possiede in $A^{\prime \prime}, B^{\prime \prime}, C^{\prime \prime}, A^{\prime}{ }_{1}, B^{\prime}, C^{\prime}$, punti multipli ordinari secondo "i numeri $n, n-i, n-i, 2 n-i, 2 n-i-j, 2 n-i-j$ rispettivamente, " ed incontra la retta $B^{\prime}{ }_{1} C^{\prime}{ }_{1}$, fuori di $B^{\prime}{ }_{1}$ e $C^{\prime}{ }_{1}$, in $j$ punti, i quali possono " coincidere e dar luogo anche ad un punto $j$-plo di $f^{\prime \prime}$."

Ad un piano $V^{\prime \prime}$ di $\Sigma^{\prime \prime}$ condotto per il punto $O^{\prime \prime}$ corrisponde in $\Sigma^{\prime}(3, \mathrm{I})$ un cono quadrico $\Gamma_{1}$, circoscritto al triedro $O^{\prime}, a_{1} b_{1} c_{1}$, il quale taglia la superficie $F^{\prime}$ secondo una curva $f_{1}$ dell'ordine $6 n-2 i-j$, che possiede in $O^{\prime}$ un punto multiplo secondo $4 n-2 i-j$ ed ha come tangenti nel punto stesso le rette d'intersezione del cono $\Gamma_{1}$ col cono tangente in $O^{\prime}$ alla superficie $F^{\prime}$. Ora questo ultimo cono si scinde nei due piani $a^{\prime} d^{\prime}$ e $b^{\prime} c^{\prime}$ contati $n$ ed $n-i$ volte rispettivamente $(18, \mathrm{I})$; e il cono $\Gamma_{1}$ passa per la retta $a_{1}$ intersezione dei piani medesimi, e sega il primo di questi in una retta $u_{1}$ e il secondo in una retta $v_{1}$. Quindi la retta $u_{1}$ e $v_{1}$ debbono essere contate rispettivamente come $n$ ed $n-i$ tangenti della curva $f_{1}$ nel punto $O^{\prime}$; eppero la retta $a_{1}$ vale per $2 n-i-j$ tangenti della medesima curva nello stesso punto. Dunque dei rami della curva $f_{\mathrm{t}}$, passanti per il punto $O^{\prime}, n$ toccano in questo punto la retta $u_{1}$, altri $n-i$ toccano la retta $v_{1}$ e i rimanenti $2 n-i-j$ toccano la retta $a_{4}$.

Al cono $\Gamma_{1}$ di $\Sigma^{\prime}$ corrisponde in $=(15$, II $)$ un cono del $4 .{ }^{\circ}$ ordine $\Gamma_{0}$, il quale possiede come doppia la retta $a$ e lungo questa viene toceata da un piano $\tau$ variabile con $\Gamma_{0}$, e dal piano fisso $O s$, il quale taglia il cono stesso in una sola retta $u_{0}$, oltre $a$. Il cono $\Gamma_{0}$ interseca la superficie data $F$ secondo una curva $f_{0}$ dell'ordine $4 n$, la quale è fornita di $n-i$ punti doppi distinti sulla retta $a$ (nei punti in cui questa incontra, fuori di $A$; la superficie $F^{\prime}$ ), e dei due rami di $f_{0}$ passanti per ciascuno di questi punti uno tocea il piano $\tau$ e l'altro il piano $O s$; possiede in $A$ un punto multiplo secondo $2 i$ e delle tangenti alla curva in questo punto $i-j$ giacciono sul piano $O s$ e sono distinte fra loro; infine si appoggia in $n$ punti in generale distinti alla retta $u_{0}$. Per quest' ultima proprietà, la curva $f_{1}$ passa per il punto $O^{\prime}$ con $n$ rami tangenti nel punto stesso alla retta $u_{1}$ e costituenti una singolarità identica a quella posseduta in $O^{\prime}$ dalla curva che si ottiene tagliando la superficie $\boldsymbol{F}^{\prime}$ con un piano condotto ad arbitrio per la retta $u_{1}$. Inoltre per la proprietà che ha la curva $f_{0}$ di possedere $n-i$ rami tangenti in altrettanti punti distinti della retta $a_{4}$ al medesimo piano $\tau$ la curva $f_{1}$ passa per $\sigma^{\prime}$ con altri $n-i$ rami tangenti nel punto stesso alla retta $v_{1}$ e costituenti una singolarità identica a 
quella ivi posseduta dalla curva che si ottiene tagliando la superficie $F^{\prime}$ con un piano condotto ad arbitrio per la retta $v_{1}$. Infine per l'altra proprietà della curva $f_{0}$ di avere $n-i$ rami tangenti in altrettanti punti distinti della retta $a$, al piano $O s$ ed $i-j$ rami tangenti in direzioni distinte nel punto $A$ allo stesso piano, la curva $f$ passa per $O^{\prime}$ con $2(n-i)+(i-j)=2 n-i-j$ rami tangenti nel punto stesso alla retta $a_{4}$ e costituenti una singolarità identiea a quella jvi posseduta dalla curva che si ottiene tagliando la superficie $F^{\prime \prime}$ con un piano condotto ad abitrio per la retta $a_{1}$.

Alla curva $f_{1}$ corrisponde in $\Sigma^{\prime \prime}$ la curva $f^{\prime \prime}$ nella quale il piano dato $V^{\prime \prime}$ taglia la superficie $F^{\prime \prime}$ e alle rette $u_{i}, v_{1}, a_{3}$ corrispondono rispettivamente le rette d'intersezione $u_{1}^{\prime}, v_{1}^{\prime}$ e $a^{0}{ }_{1}$ del piano $V^{\prime \prime}$ con i piani $a_{1}^{\prime} g^{\prime \prime}\left(r^{\prime \prime}=0\right)$, $a_{1}^{\prime} l^{\prime \prime}\left(P^{\prime \prime}=0\right)$ e $c_{1}^{\prime} b_{1}^{\prime}{ }_{1}\left(x^{\prime \prime}{ }_{1}=0\right)$.

Il cono $\Gamma_{1}$ si rappresenti sopra un piano $V_{1}$ proiettandolo da uno $M$ dei due punti, in cui esso viene incontrato dalla retta $s^{\prime}$. Cosl fra $i$ punti dei due piani $V_{1}$ e $V^{\prime \prime}$ nasce una corrispondenza birazionale.

Ad una retta del piano $V^{\prime \prime}$ corrisponde in $\Sigma^{\prime}(3$, IV) una cubica gobba, la quale passa per il punto $O^{\prime}$ e tocoa ivi la generatrice $g$ del cono $C_{1}$ corrispondente al punto d'incontro del piano $V^{\prime \prime}$ con la retta $s^{\prime \prime}$; inoltre passa per il centro $S^{\prime}$, e si appoggia in un altro punto alla retta $s^{\prime}$. Quindi la sua proiezione sopra il piano $V_{1}$ è una conica, la quale passa per i punti $S_{t}$ ed $O_{1}$, in cui questo piano incontra la retta $s^{\prime}$ e $M O^{\prime}$, e tocea in $O_{4}$ la proiezione $g_{1}$ della generatrice $g$. Dunque:

1. Alle rette del piano $V^{\prime \prime}$ corrispondono le coniche del piano $V_{1}$, passanti per i punti $S_{1}$ ed $O_{1}$ e tangenti in quest' ultimo alla retta $g_{1}$.

Una retta del piano $V_{s}$ determina un piano passante per il punto $M$, o a questo piano corrisponde in $\Sigma^{\prime \prime}$ una superficie del $3 .^{\circ}$ ordine $\varphi_{1}^{\prime}$, la quale contiene la generatrice $g^{\prime}$ del cono $C_{1}^{\prime}$ corrispondente al punto $M$. Siccome la retta $g^{\prime}$ appartiene anche al piano $V^{\prime \prime}$, così questo taglia ulteriormente quella superficie $\varphi^{\prime}$ secondo una conica, la quale passa per il punto $S^{\prime \prime}$, in cui la retta $s^{\prime \prime}$ ineontra il piano $V^{\prime \prime}$, e per il punto $O^{\prime \prime}$, in cui tocea la generatrice $g^{\prime \prime}$, ulteriore intersezione del cono $C^{\prime}$, con lo stesso piano $V^{\prime \prime}$. Dunque: $2{ }^{\circ}$ Alle rette del piano $V_{4}$ corrispondono le coniche del piano $V^{\prime \prime}$ passanti per i punti $S^{\prime \prime}$ ed $O^{\prime \prime}$ e tangenti in quest'ultimo alla retta $g^{\prime \prime}$.

Cosi si vede che la corrispondenza birazionale the ha luogo fra i punti dei due piani $V_{1}$ e $V^{\prime \prime}$ è una trasformazione quadratica.

La curva $f_{1}$, intersezione del cono $\Gamma_{1}$ con la superficie $F^{\prime}$ possiede in $M$ un punto $w^{-p t o}$, epperò viene proiettata da $M$ sopra il pianc $V_{1}$ in una 
curva $f_{2}$ dell'ordine $5 n-2 i-j$, la quale possiede in $S_{1}$ un punto $n^{-p l o}$ ed in $O_{1}$ un punto multiplo secondo $4 n-2 i-j$, in cui le tangenti sono le rette $u_{2}, v_{2}, a_{2}$, proiezione delle rette $u_{1}, v_{1}, a_{3}$, tangenti in $O^{\prime}$ alla curva $f_{1}$ e le singolarità della curva $f_{2}$ nei punti delle anzidette tangenti infinitamente vicini ad $O_{1}$, sono identiche rispettivamente a quelle che posseggono nel punto infinitamente vicino ad $O^{\prime}$ le sezioni operate nella superficie $F^{\prime}$ da un piano condotto ad arbitrio per la retta $u_{1}$, o $v_{1}$, od $a_{1}$. La curva $f^{\prime \prime}$ è la corrispondente di $f_{z}$ nell' anzidetta trasformazione quadratica, e per le proprietà della trasformazione stessa, alla singolarità che la curva $f_{2}$ possiede nel punto $O_{1}$ corrisponde una singolarità identica per la curva corrispondente $f^{\prime \prime}$, nel punto $O^{\prime \prime}$, si conclude :

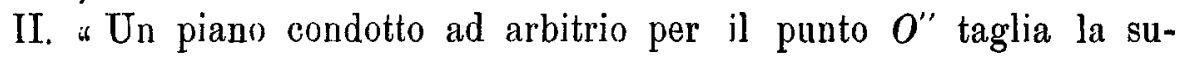
"perficie $F^{\prime \prime}$ in una curva $f^{\prime \prime}$ la quale possiede in $O^{\prime \prime}$ un punto multiplo "secondo $4 n-2 i-j$, e de' rami di questa curva passanti per $O^{\prime \prime}, n$ toc"cano il piano $a^{\prime}, g^{\prime \prime}$, col quale hanno in comune, oltre $O^{\prime \prime}$, gli stessi $n$ "punti infinitamente vicini ad $O^{\prime \prime}$; altri $n-i$ toceano il piano $a^{\prime} h^{\prime \prime}$, col "quale hanno in comune, oltre $O$ ", gli stessi $n-i$ punti infinitamente vicini " ad $O^{\prime \prime}$; infine i rimanenti $2 n-i-j$ toccano il piano $b_{1}^{\prime} c^{\prime}$, formando nel "punto infinitamente vicino ad $O^{\prime}$ una singolarità identica a quella incon"trata nel teorema IV del n. $.^{\circ} 25 . "$

Da questo teorema segue:

III. "Il cono tangente alla superficie $F^{\prime \prime}$ nel punto $O^{\prime \prime}$ si scinde " nei piani $a_{1}^{\prime} g^{\prime \prime}, a_{1}^{\prime} k^{\prime \prime}, b^{\prime}{ }_{1}^{\prime}{ }_{1}^{\prime}$ contati $n, n-i, 2 n-i-j$ volte rispetti"vamente."

Ad un piano $a^{\prime \prime}$ di $\Sigma^{\prime \prime}$ condotto ad arbitrio per la retta $a_{1}^{\prime}$ corrisponde in $\Sigma^{\prime}$ un piano $\alpha_{1}$ passante per la retta $a_{1}$ e fra $i$ punti di questi due piani ha luogo una determinata trasformazione quadratica. Il piano $\alpha_{i}$ taglia la superficie $F^{\prime}$ secondo una curva $f_{1}$ dell'ordine $3 n-i-j$, la quale $(25$, IV) possiede in $O^{\prime}$ un determinato punto multiplo dell'ordine $2 n-i-j$, in cui le tangenti coincidono con la retta $a_{1}$; ha un punto $n^{-p l o}$ ordinario nel punto in cui il suo piano $\alpha_{1}$ incontra la retta $s^{\prime}$, e $\left(24\right.$, IV) interseca la tangente $\alpha_{1}$, fuori di $O^{\prime}$, in $j$ punti. Alla curva $f_{1}$ corrisponde nell' anzidetta trasformazione quadratica, la curva $t^{\prime \prime}$ intersezione, oltre la retta $a_{1}^{\prime}$, del piano $a^{\prime \prime}$ con la superficie $F^{\prime \prime}$. Poichè la retta $a_{1}$ tangente in $O^{\prime}$ alla curva $f_{i}$ non è una retta fondamentale del piano $\alpha_{1}$ per questa stessa trasformazione, la curva $f^{\prime \prime}$ possiede in $O^{\prime \prime}$ la medesima singolarità che la curva $f_{1}$ ha in $O^{\prime}$. Inoltre alla retta $a_{1}$ di $\alpha_{1}$ corrisponde in $\alpha^{\prime \prime}$ la retta in cui questo piano taglia 
jl piano $b_{1}^{\prime} c^{\prime}{ }_{1}$; quindi come la curva $f_{1}$ incontra quella retta in soli $j$ punti fuori di $O^{\prime}$, cosi la curva $f^{\prime \prime}$ incontra questa in soli $j$ punti fuori di $O^{\prime \prime}$. Dunque :

IV. "Un piano condotto ad arbitrio per la retta $a^{\prime}$, taglia ulterior" mente la superficie $F^{\prime \prime}$ in una curva $f$ " la quale possiede in $O^{\prime \prime}$ un punto " multiplo secondo $2 n-i-j$, in cui le tangenti coincidono nella retta d'in" tersezione del piano dato col piano $b_{1}^{\prime} c^{\prime}{ }_{1}$ e la singolarità ivi risultante è "identica a quella incontrata nel teorema IV del n. ${ }^{\circ} 25$. Inoltre la curra $f$ " "incontra la sua tangente in $O^{\prime}$, oltre $i$ punti riuniti in $O^{\text {", in soli altri } j}$ " punti, i quali possono essere tutti distinti oppure tutti od in parte coin" cidenti."

In modo analogo può essere studiata la sezione fatta nella superficie $F^{\prime \prime}$ da un piano condotto ad arbitrio per la retta $b_{1}^{\prime}, 0 c^{\prime}$. E $\mathbf{E}$ poi facile vedere quali siano le sezioni operate dai piani $a_{1}^{\prime} b_{1}^{\prime}, a_{1}^{\prime} c_{1}^{\prime}, a_{1}^{\prime} l^{\prime \prime}, b_{1}^{\prime} g^{\prime \prime}, b^{\prime} h^{\prime \prime}$, $c_{1}^{\prime} g^{\prime \prime}, b_{1}^{\prime} h^{\prime \prime}$, ecc. In particolare è importante conoscere quelle ottenute con i piani tangenti ad $F^{\prime \prime}$ nel punto $O^{\prime \prime}$.

Fra questi $\nabla^{\prime}$ è il piano $a_{1}^{\prime} g^{\prime \prime}$, il quale corrisponde in $\Sigma^{\prime \prime}$ al piano $a^{\prime} d^{\prime}$ di $\Sigma^{\prime}$. Questo taglia la superficie $F^{\prime}\left(24\right.$, III) oltre le rette $a^{\prime}, d^{\prime}, a_{1}$, in altre $n-i-j$ rette concorrenti in $O^{\prime}$; quindi anche jl piano $a_{1}^{\prime} g^{\prime \prime}$ deve intanto tagliare la superficie $F^{\prime \prime}$ in $n-i-j$ rette concorrenti in $O^{\prime \prime}$. Inoltre alla retta $a^{\prime}$ di $\Sigma^{\prime}$ corrisponde in $\Sigma^{\prime \prime}$ una retta $a^{\prime \prime}$, la quale $(30,1)$ è $n$ "plo per $F^{\prime \prime}$; e questa retta $a^{\prime \prime}$, passante essa pure per $0^{\prime \prime}$, appartiene al piano $a^{\prime}{ }_{1} g^{\prime \prime}$, perchè $a^{\prime}$ appartiene al piano $a^{\prime} d^{\prime}$. Questo piano dunque taglia la superficie $F^{\prime \prime}$ in altre $n$ rette coincidenti in $\alpha^{\prime \prime}$. Infine altre $2 n-i$ ed $n$ rette $d^{\prime}$ intersezione cadono rispettivamente nelle rette $a^{\prime}$ : e $g^{\prime \prime}(28, I$ e 32 , III) passanti anche esse per $O^{\prime \prime}$. Dunque il piano $a_{1}^{\prime} g^{\prime \prime}$ taglia intanto la superficio $F^{\prime \prime}$ in

$$
(n-i-j)+n+(2 n-i)+n=5 n-2 i-j
$$

rette tutte concorrenti in $O^{\prime \prime}$; e siccome la superficie $F^{\prime \prime}$ è dell'ordine $5 n-2 i-j$, così si conclude:

V. " Il piano $a^{\prime} g^{\prime \prime}$ taglia la superficie $F^{\prime \prime}$ soltanto in rette, tutte " concorrenti nel punto $O^{\prime \prime}$."

Al piano $a^{\prime}, h^{\prime \prime}$ di $\Sigma^{\prime \prime}$ corrisponde in $\Sigma^{\prime}$ il piano $b^{\prime} c^{x}$, il quale taglia la superficie $F^{\prime}$. secondo le quattro rette $b^{\prime}, e^{\prime}, e^{\prime}, a_{1}$ di eui le prime tre sono $(n-i)^{-p l o}$ e l'ultima e $j^{-p l o}$, e in una curva $\Delta^{\prime}$ dell'ordine $2 i-j$, avente in $\sigma^{\prime}$ un punto $(i-j)^{-p t_{0}} \theta$ in $E^{*}$ un punto $i^{-p l o}(21$, VI). Fra $i$ punti dei due piani $a^{\prime} h^{\prime \prime}$ e $b^{\prime} c^{\prime}$ ha luogo una trasformazione quadratica, per la quale 
i punti fondamentali sopra $b^{\prime} c^{\prime}$ sono il punto $E^{\prime}$, il punto $O$ e il punto a questo infinitamente vicino sulla retta $h_{1}$, ulteriore intersezione del piano $b^{\prime} c^{\prime}$ con il cono $C_{3}(5, \mathrm{VI})$. In virtù di questa trasformazione alla curva $\Delta^{\prime}$ corrisponde sul piano $a^{\prime} h^{\prime \prime}$ una curva $\Delta^{\prime \prime}$ dell' ordine

$$
2(2 i-j)-[(i-j)+i]=2 i-j
$$

la quale possiede in $O^{\prime \prime}$ un punto $(i-j)^{-p t o}$, in cui le tangenti coincidono tutte con la retta d'intersezione del piano $a^{\prime}, h^{\prime \prime}$ col piano $b^{\prime} c^{\prime}$, producendo in $O^{\prime}$ per la curva $\Delta^{\prime \prime}$ una singolarità identica a quella che nel punto $O^{\prime}$ possiede la curva $\Delta^{\prime}$. La curva $\Delta^{\prime}$ incontra la tangente $a_{1}$, fuori di $O^{\prime}$, in $j$ punti, i quali possono anche coincidere e dar luogo ad un punto $j^{\text {-pto }}$ per $\Delta^{\prime}$; quindi anche la curva $\Delta^{\prime \prime}$ incontra la tangente in $0^{\prime \prime}$, fuori di questo punto, in altri $j$ punti, che, come quelli, potranno anche coincidere e dar luogo ad un punto $j^{-p l o}$ per $\Delta^{\prime \prime}$. Infine poichè il piano $b^{\prime} c^{\prime}$ contiene le due rette $b^{\prime}$ e $c^{\prime}$, il piano $a_{1}^{\prime} h^{\prime \prime}$ contiene le rette corrispondenti $b^{\prime \prime}$ e $e^{\prime \prime}$; e queste sono $(n-j)^{-p l o}$ per la superficie $F^{\prime \prime}\left(30\right.$, I). II piano $a^{\prime}{ }_{i} h^{\prime \prime}$ taglia dunque la superfocie $F^{\prime \prime}$ nello quattro rette, $a_{1}^{\prime}, h^{\prime \prime}, b^{\prime \prime} e^{\prime \prime}$, di cui la prima deve essere contata $2 n-i$ volte $(28, I)$, la seconda $n-i$ volte $(33, I)$ e ciasouna delle due rimanenti ancora $n-i$ volte; e nella curva $\Delta^{\prime \prime}$ dell'ordine $2 i-j$. Siccome $\dot{\theta}$

$$
(2 n-i)+(n-i)+(n-i)+(n-i)+(2 i-j)=5 n-2 i-j,
$$

e la superficie $F^{\prime \prime}$ è dell' ordine $5 n-2 i-j$, cos̀̀ si conelude:

VI. "Il piano $a^{\prime}, h^{\prime \prime}$ taglia la superficie $F^{\prime \prime}$ nelle quattro rette $a^{\prime}$, « $h^{\prime \prime}, b^{\prime \prime}, c^{\prime \prime}$, contate $2 n-i, n-i, n-i$ volte rispettivamente, e in una

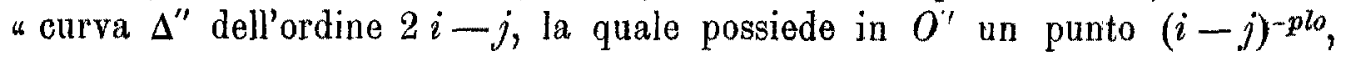
"identico a quello che ha in $O^{\prime}$ la curva $\Delta^{\prime}$ in cui le tangenti coincidono " tutte nella retta d'intersezione del piano $a^{\prime} h^{\prime \prime}$ col piano $b^{\prime \prime} c^{\prime \prime}$, e la eurva $\Delta^{\prime \prime}$ "incontra la retta medesima, fuori di $O^{\prime \prime}$, in $j$ punti, i quali possono anche " coincidere e dar luogo ad un punto $j$-plo per $\Delta^{\prime \prime}$."

Il piano $b_{1}^{\prime} c_{1}^{\prime}$ contiene le tre rette $b_{1}^{\prime}, c_{1}^{\prime}, l^{\prime \prime}$, le quali $(29$, I e $34 \mathrm{I})$ sono multiple per la superficie $F^{\prime \prime}$ secondo i numeri $2 n-i-j, 2 n-i-j$, $n-i$ rispettivamente. Il piano stesso non tocea la superficie $F^{\prime \prime}$ lungo alcuna di quelle tre rette $(29$, II e 34 , II); quindi la ineontra alteriormente secondo una eurva $\Theta$ dell' ordine.

$$
(5 n-2 i-j)-[(2 n-i-j)+(2 n-i-j)+(n-j)]=2 j .
$$

Ogni retta condotta ad arbitrio per il punto $L^{\prime \prime}$ sopra il piano $b_{1}^{\prime} c^{\prime}{ }_{i}$ deter- 
mina un piano $\sigma^{\prime \prime}$, passante per la retta $s^{\prime \prime}$, il quale $(36, I)$ taglia la superficie $F^{\prime \prime}$ secondo una curva $f^{\prime \prime}$, che incontra la retta considerata, fuori di $L^{\prime \prime}$, in $j$ punti soltanto. La retta medesima incontra la superficie $F^{\prime \prime}$, e quindi anche la curva $\Theta$, fuori di $L^{\prime \prime}$, nei medesimi $j$ punti. Dunque la curva $\Theta$ possiede in $L^{\prime \prime}$ un punto $j$-plo. Ogni retta condotta ad arbitrio per il punto $O^{\prime \prime}$ sopra il piano $b_{1}^{\prime} c_{1}^{\prime}$ determina un piano $\alpha^{\prime \prime}$ passante per la retta $a_{1}^{\prime}$, il quale taglia la superficie $F^{\prime \prime}$ secondo una curva, che (IV) incontra la retta considerata, fuori di $O^{\prime \prime}$, in $j$ punti soltanto. Quindi come nel caso precedente si conclude che la curva $\Theta$ possiede in $O^{\prime \prime}$ un altro punto $j^{-p l o}$. Il piano $\alpha^{\prime} g^{\prime \prime}$ incontra il piano $b_{1}^{\prime} c_{1}^{\prime}$ secondo una retta, la quale in virtù del precedente teorema $\mathrm{V}$, non incontra la superficie $F^{\prime \prime}$, e quindi neanche la curva $\Theta$, fuori del punto $O^{\prime \prime}$. La retta stessa tocca dunque la curva $\theta$ in $O^{\prime \prime}$, ed è l'unica tangente che la curva medesima abbia in questo punto. Raccogliendo si ha quindi :

VII. "Il piano $b^{\prime}{ }_{1} c^{\prime}{ }_{1}$ taglia la superficie $F^{\prime \prime}$ secondo una curva $\Theta$ " dell' ordine $2 j$, la quale possiede due punti $j$-plo uno in $L^{\prime \prime}$ e l'altro in $O^{\prime \prime}$, "e le tangenti in quest' ultimo punto coincidono tutte nella retta d'interse"zione dei piani $b_{1}^{\prime} c_{1}^{\prime}$ e $a_{1}^{\prime} g^{\prime \prime}{ }_{1} . "$

37. Ad un piano qualunque $U^{\prime \prime}$ di $\Sigma^{\prime \prime}$ corrisponde in $\Sigma$ una superficie del $5 .^{\circ}$ ordine $\varphi_{0}$, la quale possiede in $O$ un punto quadruplo $(15$, III), e quindi può essere rappresentata univocamente sopra un piano qualunque, mediante proiezione da $O$. Cosi fra i punti di questo piano, per il quale giova qui scegliere un piano $\sigma$ condotto ad arbitrio per la retta $s$, e quelli di $U^{\prime \prime}$ nasce una corrispondenza birazionale, nella quale sono corrispondenti due punti, che corrispondono ad uno stesso punto della superficie $\varphi_{0}$.

Una retta qualunque del piano $U^{\prime \prime}$ determina in $\Sigma^{\prime \prime}$ un piano passante per il punto $O^{\prime \prime}$, cui corrisponde in $\Sigma(15, \mathrm{II})$ un cono del $4 .^{\circ}$ ordine $\Gamma_{0}$, il quale possiede come semplici le rette $b_{0}$ e $c_{0}$ e come doppie le rette $a, b, c$, lungo la prima delle quali uno dei due piani tangenti è il piano fisso $O s$ e l'altro è un piano variabile. Quindi dicendo $B_{0}, C_{0}, B, C$ i punti in cui il piano $\sigma$ incontra le rette $b_{0}, c_{0}, b, c$, si ha:

1. ${ }^{\circ}$ Ad una retta del piano $U^{\prime \prime}$ corrisponde sul piano $\sigma$ una curva del $4 .^{\circ}$ ordine, la quale possiede come semplici i punti $B_{0}$ e $C_{0}$ e come doppi i punti $A, B, C$, nel primo dei quali una delle due tangenti è la retta fissa $s$ e l'altra è una retta variabile.

Una retta qualunque del piano $\sigma$ determina in $\Sigma$ un piano passante per il punto $O$, cui corrisponde in $\Sigma^{\prime \prime}$ un cono del $4 .^{\circ}$ ordine, il quale possiede 
d' una superficie algebrica per mezzo di trasform. biraz. dello spazio. 135

come semplici le rette $a^{\prime \prime}, b^{\prime \prime}, c^{\prime \prime}$ e come doppie le rette $a_{1}^{\prime}, b_{1}^{\prime}, c_{1}^{\prime}$. Quindi dicendo $A^{\prime \prime}, B^{\prime \prime}, C^{\prime} . A^{\prime}{ }_{1}, B_{1}^{\prime}, C^{\prime}{ }_{1}$ in cui il piano $U^{\prime \prime}$ incontra le anzidette rette, si ha:

$2 .^{\circ} \Lambda \mathrm{d}$ una retta del piano $\sigma$ corrisponde sul piano $U^{\prime \prime}$ una curva del $4 .^{\circ}$ ordine, la quale possiede come sempliei i punti $A^{\prime \prime}, B^{\prime \prime} C^{\prime \prime}$ e come doppi i punti $A_{1}^{\prime}, B_{1}^{\prime}, C^{\prime}$.

Dunque fra i punti dei due piani $\sigma$ ed $U^{\prime \prime}$ ha luogo una trasformazione del $4 .{ }^{\circ}$ ordine, la quale è il prodotto di due trasformazioni quadratiche, una delle quali può supporsi fra il piano $\sigma$ e il piano $\sigma^{\prime}$ di $\Sigma^{\prime}$, corrispondente a $\sigma$ nella trasformazione cubica fra i due spazi $\Sigma$ e $\Sigma^{\prime}$, e l'altra fra il piano $\sigma^{\prime}$ e il piano $U^{\prime \prime}$. Sul piano $\sigma^{\prime}$ i punti fondamentali per la prima delle anzidette trasformazioni quadratiche sono i punti $A^{\prime}, B^{\prime}, C^{\prime}$ in cui $\sigma^{\prime}$ incontra le tre rette $a^{\prime}, b^{\prime}, c^{\prime}$; quelli per la seconda, sono i punti $A_{1}, B_{1}, C_{1}$ in cui lo stesso piano $\sigma^{\prime}$ incontra le rette $a_{1}, b_{1}, c_{1}$.

La superficie $\varphi_{0}$ taglia la superficie data $F$ secondo una curva $f_{0}$, di cui le proprietà sono date dal teorema II del $\mathrm{n}^{\circ}{ }^{3}$ 32. Quindi è facile riconoscere che la proiezione $f_{1}$ di $f_{0}$ dal centro $O$ sopra il piano $\sigma$, è una curva dell'ordine $5 n$, la quale possiede un punto $n^{-p l o}$ ordinario in ciascuno dei due punti $B_{0}, C_{0}, G$ ed $X ; G$ ed $X$ essendo i punti in cui le rette $g$ ed $x$ incontrano il piano $\sigma$, ed $x$ la retta d'intersezione fuor delle rette fondamentali del cono $C_{0}$ col cono tangente in $O$ alla superficie $\varphi_{0} ;$ un punto $2 n$-plo ordinario in ciascuno dei punti $B$ e $C$; ed in $A$ un punto multiplo secondo $2 n+i$. Dej rami di $f_{1}$ passanti per $A, n-i$ toccano la retta $k_{1}$, intersezione del piano $k$ col piano $\sigma$, altri $(n-i)+(i-j)+2 j=n+j$ toccano la retta $s$ ed infine altri $2 i-j$ toccano altrettante rette in generale tutte distinte fra loro. Dei rami di $f$ tangenti in $A$ alla retta $-s, n-i$ sono le proiezioni degli $n-i$ rami della curva $f_{0}$ passanti per i punti $K$ della retta $a$ e tangenti nei punti stessi al piano $O s$; quindi siccome ciascuno di questi rami di $f_{0}$ ha in comune con la falda del cono $C_{0}$ tangente lungo $a$ al piano $O s$, tre punti infinitamente vicini ad $a$, tutti quegli $n-i$ rami di $f_{1}$ hanno in comune, oltre $A$, altri tre punti $A^{\prime} A^{\prime \prime} A^{\prime \prime \prime}$ infinitamente vicini ad $A$, sul ramo della curva d'intersezione $\gamma_{1}$ del cono $C_{0}$ col piano $\sigma$, che tocca in $A$ la retta $s$. Altri $i-j$ dei rami di $f_{1}$ tangenti in $A$ ad $s$, sono le proiezioni degli $i-j$ rami di $f_{0}$ tangenti in $A$ al piano $O s$; quindi siccome ciascuno di questi rami di $f_{0}$ oscula in $A$ l'anzidetta falda del cono $C_{0}$, tutti quelli $i-j$ rami di $f$ hanno in comune, oltre $A$, anche $i$ due punti $A^{\prime}$ ed $A^{\prime \prime}$. Infine i rimanenti $2 j$ rami di $f_{1}$ tangenti in $A$ ad $s$, sono le proiezioni dei $2 j$ rami di $f_{0}$ 
tangenti essi stessi in $A$ ad $s$; e siccome nessuno di questi rami di $f_{0}$ oscula in $A$ la falda del cono $C_{0}$ già considerata, così quei $2 j$ rami di $f_{1}$ hanno in comune, oltre $A$, dei tre punti $A^{\prime}, A^{\prime \prime}, A^{\prime \prime \prime}$ soltanto il primo $A^{\prime}$, il che apparirà meglio in seguito.

Alla curva $f_{\mathrm{t}}$ corrisponde sul piano $\sigma^{\prime}$, in virtù della trasformazione quadratica stabilita fra questo piano e $\sigma$, una curva $f^{\prime}$, la quale è anche la proiezione sopra $\sigma^{\prime}$ dal punto $O^{\prime}$ della curva d'intersezione $f^{\prime}$ della superficie $F^{\prime}$ con la superficie $\varphi_{1}$ di $\Sigma^{\prime}$ corrispondente al piano $U^{\prime}$. La curva $f^{\prime}$ è dell'ordine $4 n-i$, possiede in $A^{\prime}$ un punto $n^{\text {-plo }}$ ordinario e in $B^{\prime}$ e $C^{\prime}$ punti $(n-i)^{\text {phi }}$ ordinari, il che è d'accordo con il teorema II del $\mathrm{n}^{\circ} 18$ e con il teorema $I$ del $n .{ }^{\circ} 19$. Inoltre possiede punti multipli ordinari secondo i numeri $n, n$ ed $n-i$ nei punti $X_{i}, G_{i}$ ed $H_{t}$ nei quali le rette $x_{i}, g_{1}$ ed $h_{1}$ sono incontrate dal piano $\sigma^{*}$, essendo $x_{1}$ la retta corrispondente ad $x$, ed il punto multiplo ordinario in $H_{\mathrm{f}}$ provenendo dal punto $(n-i)^{-p l o}$ che la curva $f_{\mathrm{t}}$ possiede nel punto di $k_{1}$ infinitamente vicino ad $A$. Infine la stessa curva $f^{\prime \prime}$ incontra la retta $B^{\prime} C^{\prime}$ in altri $2 i-j$ punti, corrispondenti ai $2 i-j$ rami di $f_{t}$ tangenti in $A$ ad altrettante rette distinte. Quindi ha in comune con la retta $B^{\prime} C^{\prime}$, fuori dei punti $B, C^{\prime}$ ed $H_{1}$ altri

$$
4 n-i-[(n-i)+(n-i)+(n-i)+(2 i-j)]=n+j
$$

punti, i quali corrispondono ai rimanenti

$$
(2 n+i)-[(n-i)+(2 i-j)]=n+j
$$

rami della curva $f_{1}$ tangenti in $A$ alla retta $s$. Quindi quegli $n+j$ punti cadono tutti nel punto $A_{1}$ in cui la retta $a_{1}$ incontra il piano $\sigma^{\prime}$.

Alla curva $f^{\prime}{ }_{1}$ di $\sigma^{\prime}$ corrisponde sul piano $U^{\prime \prime}$, in virtù della trasformazione quadratica per la quale si passa da $\sigma^{\prime}$ ad $U^{\prime \prime}$, la curva $f^{\prime \prime}$ in cui quest'ultimo piano incontra la superficie $F^{\prime \prime}$. Intanto ai punti $X_{1}, G_{1}, H_{1}$ di $\sigma^{\prime}$ corrispondono $i$ punti in cui il piano $U^{\prime \prime}$ incontra le rette $s^{\prime \prime}, g^{\prime \prime}, h^{\prime \prime}$; e poichè quei punti non sono fondamentali per il piano $\sigma^{\prime}$, le medesime singolarita in essi possedute dalla curva $f^{\prime}{ }^{\prime}$, sono possedute dalla curva $f^{\prime}$ nei punti corrispondenti, ciò che è in accordo con i teoremi: I del n..$^{\circ} 31$, III del n. ${ }^{\circ} 32$ ed I del n. 33. Inoltre alle tangenti della curva $f^{\prime}$, nel punto $A_{1}$ corrispondono i punti d'incontro della curva $f^{\prime \prime}$ con la retta $B_{1}^{\prime} \otimes C_{1}^{\prime}$, fuori dei punti fondamentali $B^{\prime}{ }_{1}$ e $C^{\prime}{ }_{1}$. Ciascuno di questi è multiplo per $f^{\prime \prime}$ secondo $2 n-i-j$ $(29, \mathrm{I})$; quindi gli ulteriori punti d'incontro di $f^{\prime \prime}$ con l'anzidetta retta è

$$
(5 n-2 i-j)-2(2 n-i-j)=n+j \text {. }
$$


Di questi, $2 j$ sono $i$ punti d'intersezione $\theta$ della curva $\Theta$ con la retta $B_{1}^{\prime} C^{\prime}{ }_{1}$, i quali punti, per le proprietà di questa curva $(36, \mathrm{VII})$ sono tutti distinti dal punto $L$, in cui il piano $U^{\prime \prime}$, e quindi anche la retta $B^{\prime}{ }_{1} C^{\prime}$, incontra la retta $l^{\prime \prime}$. Nel punto $L$ cadono dunque:

$$
(n+j)-2 j=n-j
$$

punti d'intersezione della curva $f^{\prime \prime}$ con la retta $B^{\prime}{ }_{1} C^{\prime}{ }_{1}$, il che è in accordo col teorema I del n. ${ }^{\circ} 34$. Poichè i $2 j$ punti $\theta$ sono tutti distinti dal punto $L$, $2 j$ delle tangenti in $A_{4}$ alla curva $f_{1}^{\prime}$ debbono essere distinte dalle $n-j$ rimanenti. Al cono $C_{0}$ di $\Sigma$ corrisponde in $\Sigma^{\prime}$ il cono $C_{1}$; quindi alla curva $\gamma_{0}$, intersezione di $C_{0}$ col piano $\sigma$, corrisponde sopra $\sigma^{\prime}$ la curva $\gamma_{1}$ secondo oui questo piano taglia il cono $C_{1}$; epperò ai punti $A^{\prime}, A^{\prime \prime}, A^{\prime \prime \prime}$ di $C_{0}$ infinitamente vicini ad $A$ corrispondono rispettivamente il punto $A_{1}$ e $i$ punti $A_{1}^{\prime}$ e $A^{\prime \prime}{ }_{1}$ infinitamente vicini ad $A_{1}$ sulla conica $\gamma_{1}$. La curva $f_{1}$ passa per il punto $A^{\prime \prime}$ con $(n-i)+(i-j)=n-j$ rami, dei quali $n-i$ soltanto passano ancora per il punto $A^{\prime \prime \prime}$; quindi la curva $f_{1}^{\prime}$ passa con $n-j$ rami per $A^{\prime}$, e con $n-i$ rami per $A^{\prime \prime}{ }_{1}$, e per l'osservazione dianzi fatta sulle tangenti in $A_{1}$ alla curva $f^{\prime}{ }_{1}$, le tangenti coincidenti con la retta $A_{1} A^{\prime}$, sono soltanto le $n-j$ tangenti agli anzidetti $n-j$ rami di $f_{1}^{\prime}$. Quindi con la trasformazione quadratica mediante la quale si passa dal piano $\sigma^{\prime}$ al piano $U^{\prime \prime}$; la singolarita che la curva $f_{;}^{\prime}$ possiede in $A^{\prime}$, si cambia in un punto $(n-j)^{-p l o}$ per la curva $f^{\prime \prime}$. Questo punto cade in $L$, perchè al piano di $\Sigma^{\prime}$ tangente al cono $C_{1}$ lungo la retta $a_{4}$ corrisponde in $\Sigma^{\prime \prime}$ il piano $a_{1}^{\prime} l^{\prime \prime}$, ed $i--j$ delle tangenti ad $f^{\prime}$ nel punto stesso sono distinte fra loro, mentre le rimanenti coincidono nella retta d'intersezione del piano $U^{\prime \prime}$ con il piano $O^{\prime \prime} s^{\prime \prime}$, essendo questo il piano di $\Sigma^{\prime \prime}$ corrispondente al cono $C_{1}$. Inoltre gli $n-i$ rami di $f^{\prime \prime}$ che toccano in $L$ l'anzidetta retta hanno in comune con la retta medesima $n-i$ punti riuniti nel punto infinitamente vicino ad $L$. Cosi mentre si ha una riconferma del teorema II del $\mathrm{n}^{\circ} 34$, si trova ancora:

I. "La sezione fatta nella superficie $F^{\prime \prime}$ mediante un piano con" dotto ad arbitrio per un punto generico $L$ della retta $l$, è una curva che " possiede in $L$ un punto $(n-j)^{-p l o}$ ed $i-j$ de'suoi rami passanti per $L$ " hanno le tangenti tutte distinte fra loro, mentre $i$ rimanenti $n-j$ rami "toccano tutti la retta d'intersezione del piano considerato col piano $O^{\prime \prime} s^{\prime}$, "dando luogo ad un punto $(n-i)^{- \text {plo }}$ per $f$ infinitamente vicino ad $L$."

Supponendo che il piano $U^{\prime \prime}$ passi successivamente per i punti $G^{\prime \prime}, H^{\prime \prime}, L^{\prime \prime}$, come il teorema che precede si dimostrano gli altri :

Annali di Matematica, tomo XXV. 
138 Pannelli: Sulla riduzione delle singolarità d'una superficie, ecc.

II. "La sezione fatta nella superficie $F$ " mediante un piano con"dotto ad arbitrio per il punto $G$ ", è una curva che possiede in $G$ " un " punto $n^{-p l o}$ e i suoi rami passanti per $G^{\prime \prime}$ toccano tutti la retta d'interse-

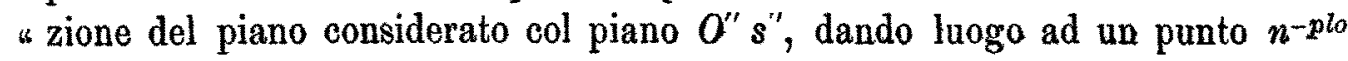
" infinitamente vicino a $G^{\prime \prime}$."

III. " La sezione fatta nella superficie $F$ " mediante un piano con" dotto ad arbitrio per il punto $H^{\prime}$, è una curva che possiede in $H^{\prime \prime}$ un "punto $n^{-p l o}$ e de' suoi rami passanti per $H^{\prime}, i$ toccano altrettanti rette di"stinte e i rimanenti $n-i$ toccano la retta d'intersezione del piano consi"derato col piano $O^{\prime \prime} s$ ", dando luogo ad un punto $(n-i)^{- \text {plo infinitamente }}$ "vicino ad $H^{\prime \prime}$."

IV. "La sezione fatta nella superficie $F^{\text {" }}$ mediante un piano con" dotto ad arbitrio per il punto $L^{\prime \prime}$, è una curva che passiede in $L^{\prime \prime}$ un " punto $n^{-p l o}$ e de' suoi rami passanti per $L^{\prime \prime}, j$ toccano altrettante rette, che " possono essere tutte distinte, oppure tutte od in parte coincidenti e i rima" nenti $n-j$ toccano la retta d'intersezione del piano considerato col piano " $O^{\prime \prime} s^{\prime}$, dando luogo a due punti, uno $(n-j)^{-p l o}$ e l'altro $(n-i)^{-p l o}$, con"secutivi ad $L^{\prime \prime}$ su quest'ultima tangente. "

Con questi teoremi lo studio della superficie $F^{\prime \prime}$ è compiuto, e se la curva $\Theta\left(36\right.$, VII) è una curva semplice, nel qual caso essa possiede in $L^{\prime \prime}$ un punto $j$-plo ordinario, la riduzione della singolarità posseduta nel punto $A$ dalla superficie $F$, ̀̀ effettuata. Ma la curva $\Theta$ può scindersi in parti, di cui ciascuna sia multipla per $\vec{F}^{\prime \prime}$, oppure ridursi ad una conica $j^{-p l a}$ di natura ignota. Si studierd in un' altra Memoria la trasformazione che in questi casi conviene applicare ancora alla superficie $F^{\prime \prime}$.

Pavia, gennaio 1897. 